\title{
$\checkmark||||||||$
}

AEC RESEARCH AND

DEVELOPMENT REPORT

\section{MATERIAL BUCKLING MEASUREMENTS ON GRAPHITE-URANIUM SYSTEMS AT HANFORD: \\ A SUMMARY TABULATION}

MASTER

HW-69525

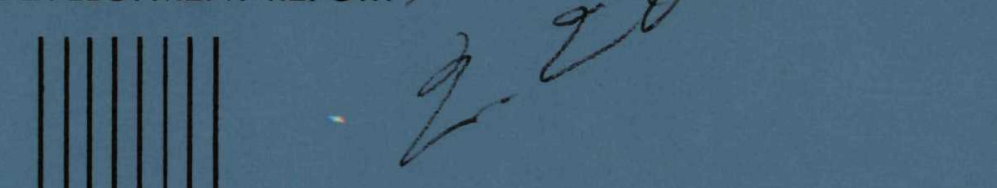

D. E. WOOD

MAY, 1961

HANFORD ATOMIC PRODUCTS OPERATIO'N RICHLAND, WASHINGTON

GENERAL Gே ELETRIC 


\section{LEGAL NOTICE}

This report was prepared as an account of Government sponsored work. Neither the United States, nor the Commission, nor any person acting on behalf of the Commission:

A. Makes any warranty or representation, expressed or implied, with respect to the accuracy, completeness, or usefulness of the information contained in this report, or that the use of any information, apparatus, method, or process disclosed in this report may not infringe privately owned rights; or

B. Assumes any liabilities with respect to the use of, or for damages resulting from the use of any information, apparatus, method, or process disclosed in this report.

As used in the above, "person acting on behalf of the Commission" includes any employee or contractor of the Commission, or employee of such contractor, to the extent that such employee or contractor of the Commission, or employee of such contractor prepares, disseminates, or provides access to, any information pursuant to his employment or contract with the Commission, or his employment with such contractor. 
$\mathrm{HW}-69525$

UC-34, Physics

(TID-4500, 17th Ed.)

MATERIAL BUCKLING MEASUREMENTS

ON GRAPHITE-URANIUM SYSTEMS AT HANFORD:

A SUMMARY TABULATION

By

D. E. Wood

Nuclear Physics Research

Physics and Instrument Research and Development

Hanford Laboratories

May, 1961

\title{
HANFORD ATOMIC PRODUCTS OPERATION RICHLAND, WASHINGTON
}

\begin{abstract}
Work performed under Contract No. AT (45-1)-1350 between the Atomic Energy Commission and General Electric Company
\end{abstract}

Printed by/for the U. S. Atomic Energy Commission

Printed in USA. Price \$2.25. Available from the Office of Technical Services

Department of Commerce

Washington 25, D.C. 


\section{DISCLAIMER}

This report was prepared as an account of work sponsored by an agency of the United States Government. Neither the United States Government nor any agency Thereof, nor any of their employees, makes any warranty, express or implied, or assumes any legal liability or responsibility for the accuracy, completeness, or usefulness of any information, apparatus, product, or process disclosed, or represents that its use would not infringe privately owned rights. Reference herein to any specific commercial product, process, or service by trade name, trademark, manufacturer, or otherwise does not necessarily constitute or imply its endorsement, recommendation, or favoring by the United States Government or any agency thereof. The views and opinions of authors expressed herein do not necessarily state or reflect those of the United States Government or any agency thereof. 


\section{DISCLAIMER}

Portions of this document may be illegible in electronic image products. Images are produced from the best available original document. 


\section{ABSTRACT}

This report summarizes measurements of material bucklings for graphite-uranium systems at Hanford. It provides a comprehensive listing and a guide to the original data sources. Complete information on physical and nuclear properties of the lattice and the geometry of the exponential assembly is included, along with some of the auxiliary data taken.

The fuel sizes vary from 0.925 to 2.5 inches diameter for five different fuel geometries. The lattice spacings vary from 4-3/16 to 15 inches. Over 300 measurements of material buckling are included. 


\section{TA'BLE OF CONTENTS}

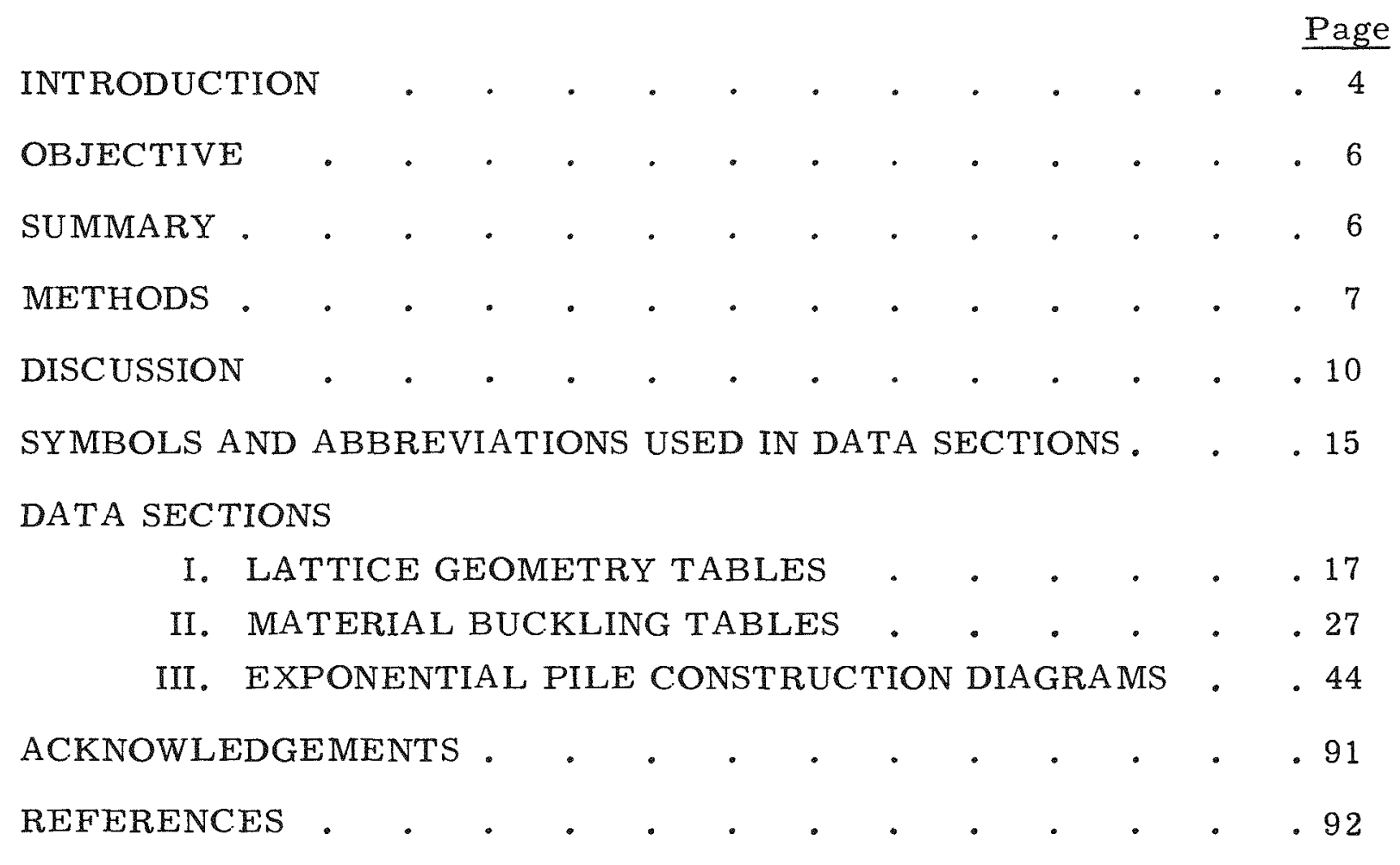


MATERIAL BUCKLING MEASUREMENTS

ON GRAPHITE-URANIUM SYSTEMS AT HANFORD:

A SUMMARY TABULATION

\section{INTRODUCTION}

Exponential measurements of material bucklings for graphiteuranium lattices have been in progress since the earliest days of the Nuclear Age. The classic experiments of the Manhattan Project have been summarized by Davenport. (7) These experiments, started in 1941, lead to the selection of the original Hanford lattice, and aided in confirming the postulate that a self-sustaining chain reaction could be created using natural uranium. These measurements are primarily of historical interest since later studies not only have covered tha same range in more detail, but also have used graphite and uranium of higher purity. A new series of experiments was started in 1951 in connection with new reactor construction at Hanford to provide a better lattice for plutonium production and to increase the available knowledge of lattice constants. This new series has been continued and extended to larger fuel sizes and to complex fuel geometries. Although many other techniques for obtaining lattice data have been developed, the exponential method still has enough advantages to make buckling measurements worthwhile. The main refinement in present day exponential experiments is the tendency to use exponentials to obtain more information than the basic material buckling, such as control rod strengths, temperature effects, multiregion problems, and mixed fuel loadings.

The time scale of the various measurements is shown in Figure 1. The period of measurements on each fuel type and enrichment is shown without a further breakdown into the individual lattice spacings covered. Measurements were not necessarily made over the full time span.

Most of the measurements are reported in formal documents or publications (see References), although some are available only in Quarterly or Monthly Reports, and a few can be found only in the original notebooks. 


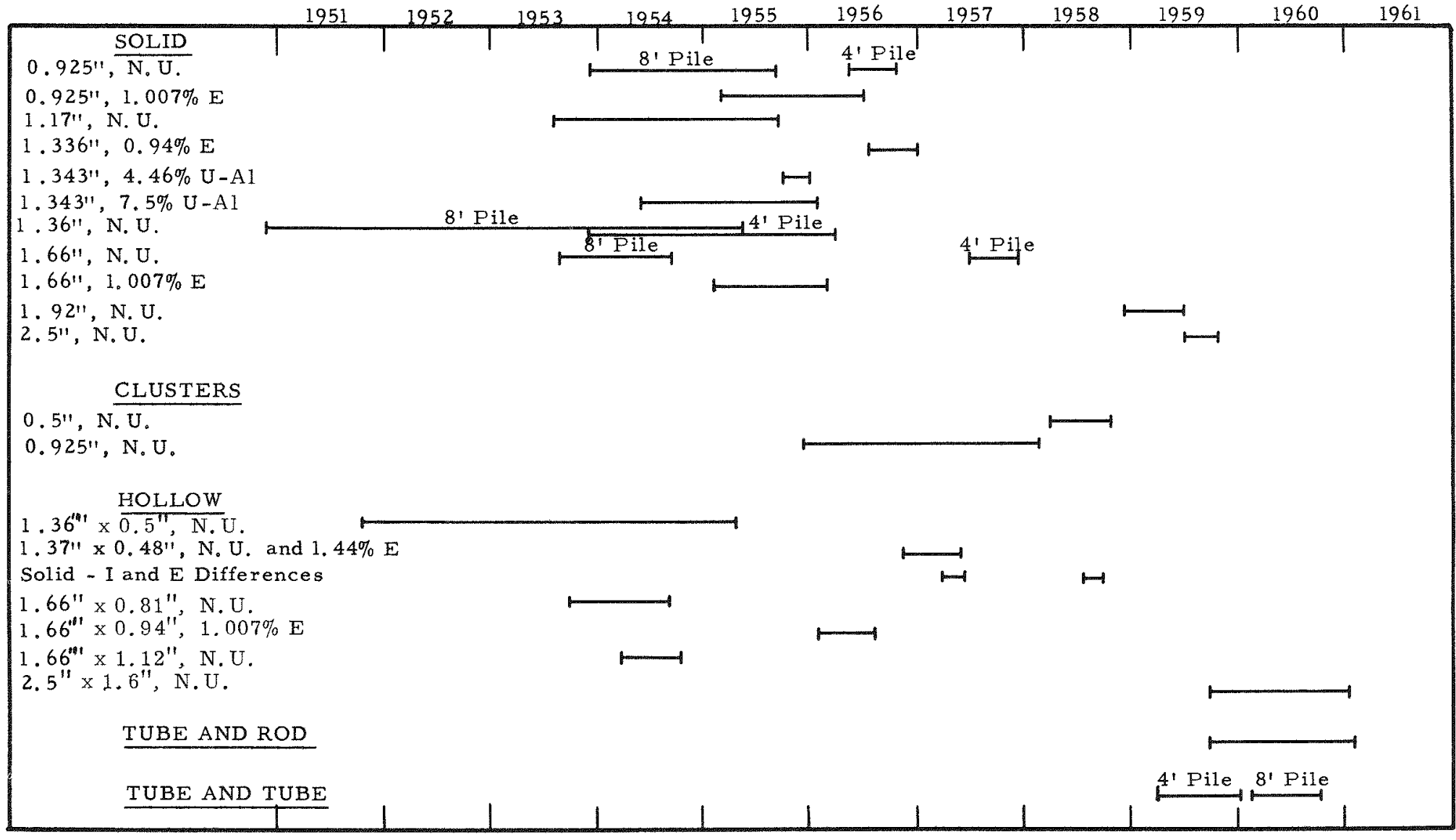

\section{FIGURE 1}

Time Span for Exponential Experiments at Hanford 
The primary references are Davenport ${ }^{(7)}$ (solid rods up to 1.66 -inch diameter in 8-foot piles), Block and Richey ${ }^{(28)}$ (1.66-inch rods in 4-foot piles), Endres and Wood ${ }^{(35)}$ (1.92- and 2.5-inch rods in 4-foot piles), Endres and Block $^{(32)}\left(0.5\right.$-inch clusters), Block ${ }^{(30)}$ (0.925-inch clusters), Clayton ${ }^{(4)}$ (hollow rods), Clayton ${ }^{(6)}$ (enriched fuel), Endres and Wood ${ }^{(37)}$ (2.5-inch internally moderated fuel), and Endres and Wood ${ }^{(39)}$ (tube-and-tube fuel in 8 -foot piles). The original references should be consulted for detailed study of the measurements since all details and methods are not included here. Furthermore, the different reports of the same measurements do not necessarily agree, either because of misprints or a later refined analysis of the data. In the present work an attempt has been made to record the best values of the physical and nuclear parameters, but success in this attempt can not be guaranteed.

\section{OBJECTIVE}

This report is written to provide a listing of buckling measurements on graphite-uranium lattices at Hanford in tabular summary form. Over 300 buckling measurements have been made covering a wide range of fuel sizes and geometries, lattice spacings, enrichments, and coolant fractions. The last previous summaries were written in $1956,(7,11)$ so a reasonably current listing is needed to reduce the need for constant reference to the widely scattered reports of individual measurements. It is intended that this summary shall be complete enough so that future work will require only the addition of a few tables to the present summary rather than a completely new listing.

\section{SUMMARY}

Buckling measurements on graphite-uranium systems at Hanford are listed in tabular form for a wide range of experimental parameters. Fuel diameters from 0.925 to 2.5 inches, lattice spacings from $4-3 / 16$ to 15 inches, fuel geometries from solid rods to two concentric tubes, enrichments from natural uranium to 1.44 per cent enriched uranium (and a few fully enriched fuels alloyed with aluminum), and several coolant fractions 
of light water, air, and occasionally monoisopropylbiphenyl, have been studied in the program summarized here. The sizes of exponential piles have varied from 4 feet square to 8 by 10 feet with the height from 7 to 10 feet.

The bucklings are reported along with a wide variety of physical and geometrical information for the lattices and piles. Additional experimental parameters included are the cadmium ratio, extrapolation distance (distance beyond the physical pile edge where the analytic continuation of the flux goes to zero), and the standard deviations of the measured values. Some of these parameters are not available for all piles.

The tabulation is restricted to buckling measurements and does not include results of fine structure measurements which were made in many of the earlier studies. Other lattice parameters such as $L^{2}, k_{\infty}$, and $f$ are similarly excluded. Measurements of diffusion length have been evaluated by Lee; ${ }^{(8)}$ other lattice parameters are not yet available in summary form. Earlier diffusion length studies are reported by Block, (1) and a measurement of buckling as a function of temperature is reported by Lloyd and Richey. (9)

Wherever possible, reference to documents or articles containing the data are included as well as the numbers of the original notebooks containing processed data.

\section{METHODS}

The experimental techniques are detailed in the references and are discussed here only where methods were changed between the various sets of measurements.

The basic pile is an 8-foot cube of the lattice supported by a graphite base containing the neutron sources. A smaller pile ( 4 feet long, 4 to 6 feet wide, and 6 to 8 feet high) is used whenever the amount of material available is limited or the buckling is so large that a critical assembly would be formed. When the available fuel is inadequate even for a 4-foot pile, a "core" or "buffer" method is used. In the "core" method, an array 
of the test lattice is surrounded on four sides by different fuel in the same lattice spacing. Measurements in the core region then are used to determine the buckling. In the "buffer" method, one or two layers of different fuel are used at the top and bottom of the lattice but not at the sides. Either method is accurate if both the buckling and cadmium ratio are matched for the test lattice and for the buffer. The configuration of the core and buffer experiments are indicated in the appropriate diagrams of Section III.

The placement of the neutron sources has varied somewhat to suit the requirements of the experiments. In the early experiments the four sources were placed just below the lattice region at the nodes of the third harmonic $(X= \pm a / 4, \cdot Y= \pm b / 4)^{*}$ to minimize the harmonic corrections. Separate traverses were used to correct for various sources of background. Later the sources were lowered about halfway down the base, and the "cadmium shutter" traverse was used to correct for background. The cadmium shutter is placed in slots between the base and the exponential region. Early in 1957, traverses in the 4 -foot piles indicated that fast neutrons leaving the base were scattering back into the exponential region. The sources then were moved to within 1 inch of the vertical center line (the "clustered" source position) to minimize leakage. Later studies ${ }^{(39)}$ indicated that the effective pile size is different for the split and clustered source positions when the traverses are analyzed with thermal source theory.

The deficiency of the thermal source theory is the assumption that neutrons are born at thermal energies at the source position. The fast neutrons actually produce a diffuse source of thermal neutrons. The harmonic corrections thus are based on an incorrect source condition unless the diffuse source is considered.

The effective pile size is defined as the physical size plus twice the extrapolation distance, $\lambda$. For the 8 -foot pile measurements a series of horizontal traverses ${ }^{(3)}$ gave an average value of 1 inch for $\lambda$. The largest lattice spacing used in this study was $8-3 / 8$ inches. When 4 -foot pile

* This location is called the "split" source position. 
measurements were started several known lattices were measured and the value of $\lambda$ was determined by the effective size required to give the same buckling from both 4-foot and 8-foot piles. Horizontal traverses aiso were taken. Both methods indicated that a slightly larger value of $\lambda, 1.15$ inches, was appropriate for the 4-foot piles. When the clustered sources were used for the 4-foot pile measurements with 1.66-inch solid rod, the comparison of bucklings for 8-foot and 4-foot piles indicated that the value of $\lambda$ should be 1.66 inches. All measurements on the larger fuels (1.92 and 2.5 inches) included values of $\lambda$ for each lattice. The traverses parallel to the fuel columns indicated that $\lambda_{1}$, was constant at 1.03 inches, but traverses perpendicular to the fuel showed that $\lambda_{\perp}$ had to be measured for each lattice. Errors in buckling due to errors in $\lambda$ for various pile sizes are listed in Table $I$ as an indication of the importance of precise knowledge of $\lambda$, especially for the smaller piles.

\section{TABLE I}

\section{ERRORS IN BUCKLING FROM UNCERTAINTIES IN $\lambda$}

Pile Width or Length (feet)
Uncertainties in $\lambda$ (inches)

\begin{tabular}{lrrrrr}
\hline 0.05 & 0.1 & 0.2 & 0.3 & 0.5 & 1.0 \\
& \multicolumn{5}{c}{ Error ${ }^{*}$ in Buckling $\left(10^{-6} \mathrm{~cm}^{-2}\right)$} \\
\\
2.7 & 5.5 & 11.0 & 16.5 & 27.6 & 55.1 \\
1.4 & 2.8 & 5.6 & 8.4 & 14.1 & 28.2 \\
0.8 & 1.6 & 3.3 & 4.9 & 8.2 & 16.4 \\
0.5 & 1.0 & 2.1 & 3.1 & 5.2 & 10.3 \\
0.3 & 0.7 & 1.4 & 2.1 & 3.5 & 6.9 \\
0.15 & 0.3 & 0.7 & 1.1 & 1.5 & 3.5
\end{tabular}

* The error in buckling includes the effect of the uncertainty in $\lambda$ on each side of the pile, and the error should be applied separately to the width or length of the pile. 


\section{DISCUSSION}

The data available on graphite-uranium exponential measurements at Hanford have been divided into three sections covering lattice geometry, nuclear parameters, and details of pile construction.

Section I on lattice geometry is arranged according to fuel geometry with a table of uranium dimensions and a table of cell dimensions for each fuel type. The fuel diameter is listed in inches since this is the normal method of referring to the various fuels. All other dimensions are in centimeters. The solid and equivalent densities of the uranium are listed. The equivalent density is the result obtained if the uranium is assumed to fill the end cap space without changing the radius. The surface-to-mass ratios are calculated from the solid density. Figures 2 and 3 are pictures of some of the fuels used. Dimensions of the aluminum, coolant, and graphite are listed in the second table for each type of fuel. The process tube inside radius includes the fuel supporting ribs (if any), which are assumed to be distributed uniformly on the inside of the process tube. The cross-sectional a reas of all components and the total aluminum-touranium volume ratio are recorded. The total aluminum volume and aluminum-to-uranium ratio include the end caps which are assumed to be distributed uniformly over the length. The volume of water usually is measured and does not necessarily agree with the aluminum dimensions.

The aluminum used is Type $2 \mathrm{~S}$ (99.2 per cent aluminum) with a nominal density of 2.7 . The graphite purity is indicated by the diffusion length (perpendicular to the extrusion axis) of $54.2 \mathrm{~cm}$ referred to a standard density of 1.60. The graphite density is 1.647 for lattice spacings of $7,7-1 / 2,8,14$, and 15 inches, and 1.658 for all others. Atomic ratios are derived using atomic densities of $0.04783 \times 10^{24}$ atoms $/ \mathrm{cc}$ for uranium at density $18.9,0.0602 \times 10^{24}$ atoms/cc for aluminum at density $2.7,0.0335 \times 10^{24}$ molecules/cc for water at density 1.0 , and 0.0803 x $10^{24}$ atoms/cc for graphite at density 1.60 .

The outside radius of the graphite is determined from the area equivalent to the square or rectangular lattice and listed in Table I-6 for all lattice spacings used. 


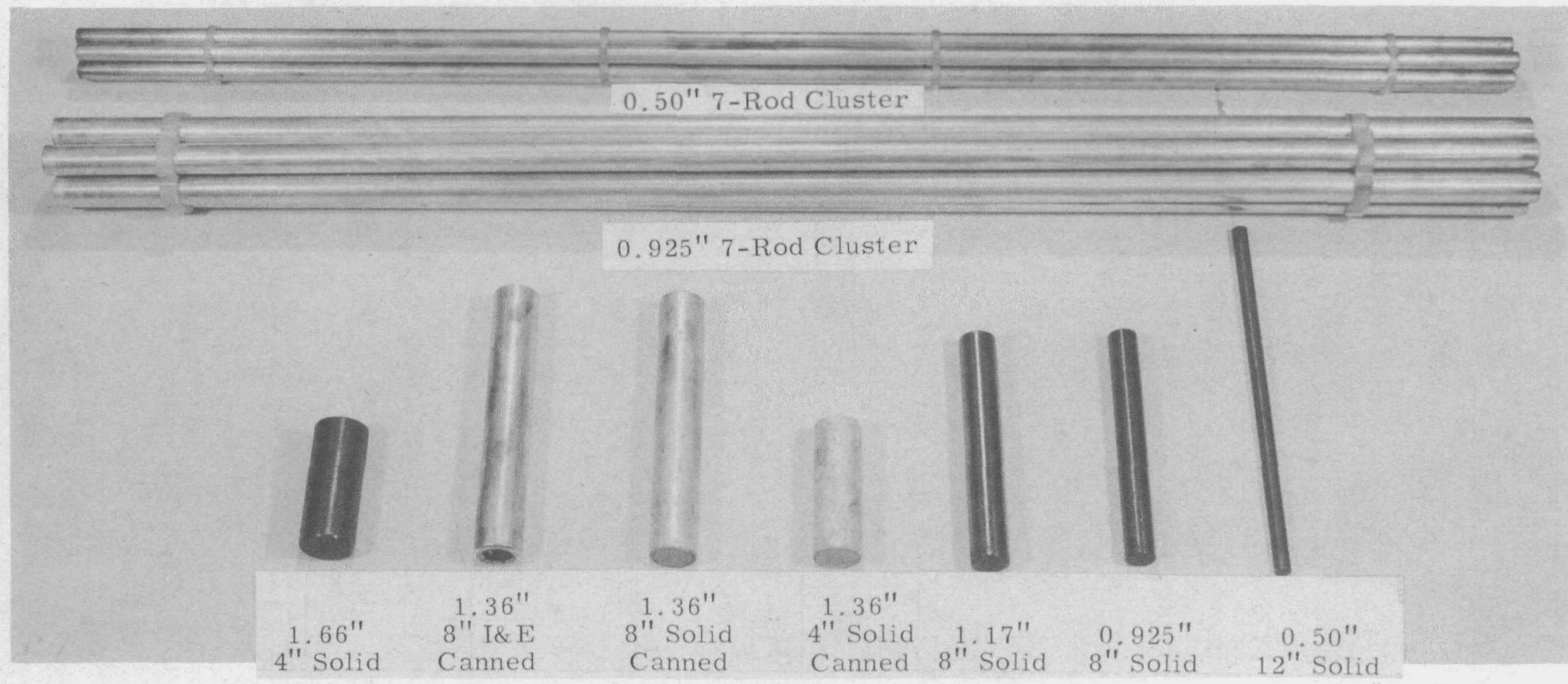

FIGURE 2

Fuel Elements Used in Exponential Experiments 


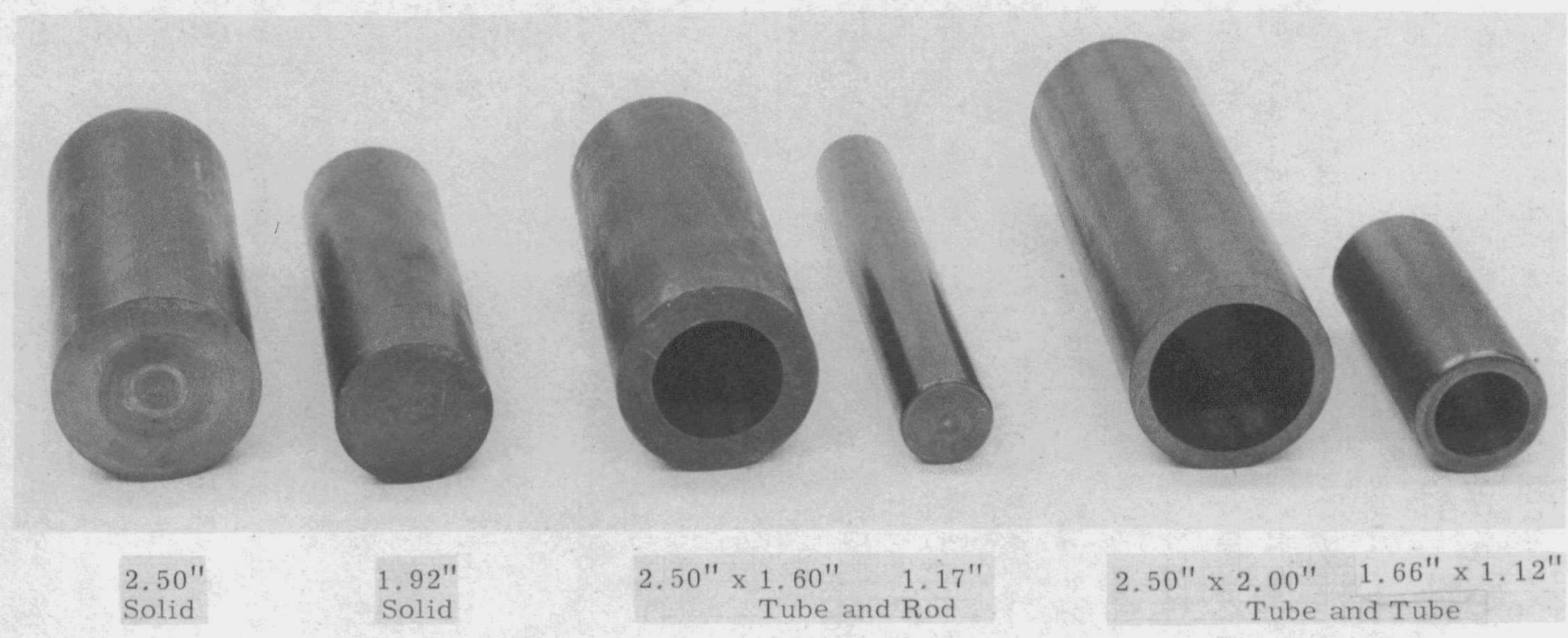

FIGURE 3

装

$\sum$

os

Large Fuel Elements Used in Exponential Experiments 
Section II containing the nuclear parameters is arranged in the same order as Section I. A separate table for each fuel size and enrichment lists the material bucklings and cadmium ratios versus lattice spacing for each aluminum-to-uranium ratio. The aluminum-to-uranium ratios are in the same order in both sections. Volume and atom ratios of aluminum-, water-, and carbon-to-uranium are listed, along with the approximate completion date of the measurement. Where applicable, the reference reporting the results is included together with the number of the notebook where the processed data and details are stored. All notebooks are stored in the Exponential Pile Laboratory at the present time. The cadmium ratios were measured with a $\mathrm{BF}_{3}$ tube and were always taken at the edge of the cell on a line between centers of fuel elements.

The $\mathrm{B}_{11}$ listed is the vertical relaxation length determined from a least squares fit to the corrected count rates. The analysis is limited to the region where the cadmium ratio is essentially constant. The buckling then is related to the $B_{11}$ through the effective pile size, which is found by adding twice the extrapolation distance to the physical pile size. Recent measurements include the measured value of $\lambda$, the cell position where the traverse was taken, and the calculated standard deviations in $\lambda$ and $\mathrm{B}^{2}$. The cell position of the horizontal traverse is keyed to the cell diagram in Figure II-1.

The first part of the third section on pile geometry is a list of lattice spacings used for each fuel. The front face diagram then is given for each different kind of pile. Several piles are combined with appropriate footnotes wherever possible. Counting slot positions, source positions, and pile dimensions are tabulated with the diagrams. Pictures of a few of the piles are included. Since most of the bases are similar, only a few of them are shown. Counter slots are shown by smaller circles than fuel.

The precision of the measurements is not the same for all sets of measurements. The 4-foot pile measurements are survey type studies intended to find relative values, but not necessarily absolute values of the buckling. The extrapolation distances are not too well known for the early 
4-foot piles, so only the relative values are significant. The larger lattice spacings ( $>8-3 / 8$ inches) in the 8 -foot piles did not include measurements of $\dot{\lambda}$ and may not be as precise as the smaller lattice spacings. However, the 8 -foot piles are large enough so that an error in $\lambda$ would not be very serious. The use of clustered sources now is known to result in larger values of $\lambda$, and older measurements with clustered sources have not been corrected for this effect. In general the best values of $\mathrm{B}^{2}$ come from 8 -foot piles with split sources and an assumed $\lambda$ of 1 inch. This value of $\lambda$ was confirmed by an extensive series of horizontal traverses with split sources. The error expected in these measurements is about $4 \times 10^{-6}$ $\mathrm{cm}^{-2}$, which consists of independent errors of 2 to $3 \times 10^{-6} \mathrm{~cm}^{-2}$ from the vertical traverse and an uncertainty in $\lambda$ of 0.3 inch in each direction. The next best values of $\mathrm{B}^{2}$ come from smaller piles ( 4 to 8 feet) with measured values of $\lambda$ for every configuration. Here the usual errors are 3 to $5 \times 10^{-6} \mathrm{~cm}^{-2}$ in the vertical traverse, 0.1 inch in $\lambda$ parallel to the process tube, and 0.3 inch in $\lambda$ perpendicular to the process tubes. The combined error is around $10 \times 10^{-6} \mathrm{~cm}^{-2}$ in $\mathrm{B}^{2}$. The errors for other situations can be derived from recent studies of variation in the extrapolation distance and the effects of the position of the source. The evaluation of these systematic exrors is not yet complete. 
SYMBOLS AND ABBREVIATIONS USED IN DATA SECTIONS

N.U.

$\mathrm{V}-\mathrm{Al} / \mathrm{U}, \mathrm{V}-\mathrm{H}_{2} \mathrm{O} / \mathrm{U}, \mathrm{V}-\mathrm{C} / \mathrm{U}$

$\mathrm{A}-\mathrm{Al} / \mathrm{U}, \quad \mathrm{A}-\mathrm{H}_{2} \mathrm{O} / \mathrm{U}, \mathrm{A}-\mathrm{C} / \mathrm{U}$

$\mathrm{S}$ and $\mathrm{U}$

$\mathrm{S} / \mathrm{M}$

I, E, I and E

$\rho$

Pe

L.S.

$\mathrm{B}_{11}$

$\mathrm{CR}$

$\mathrm{D}, \mathrm{W}, \mathrm{M}$

$B^{2}$

Ref.

N.B.

$\mathrm{NB}$

$N_{\lambda}$

$\lambda_{\|}, \lambda_{1}$

$\mathrm{L}_{\lambda}$
Natural uranium

Volume ratios of aluminum, water, and carbon to uranium

Atom (or molecule) ratios of aluminum, water, and carbon to uranium

Separated and unseparated, referring to the rods in a cluster fuel assembly

Surface-to-mass ratio in $\mathrm{cm}^{2} / \mathrm{gram}$, using actual density, before end cap correction.

Coolant location referring to hollow fuel: internal, external, or internal and external coolant

Density, grams $/ \mathrm{cm}^{3}$

Equivalent density corrected for end caps, grams $/ \mathrm{cm}^{3}$

Lattice spacing, inches

Vertical relaxation length, $\mathrm{cm}$

Cadmium ratio for a $\mathrm{BF}_{3}$ proportional counter at the center of the cell edge.

Coolant: air, water, or monoisopropylbiphenyl

Material buckling, $10^{-6} \mathrm{~cm}^{-2}$

Reference document or publication

Reference notebook

Number of points fitted to obtain $\mathrm{B}_{11}$

Number of points fitted to obtain $\lambda$

Extrapolation distance, parallel and perpendicular to the process tubes, as measured by the argument of the fitted cosine less the physical pile size, in $\mathrm{cm}$. The number listed is the average of both sides.

Location in the cell of the horizontal traverse (see Figure II-1, page 43). 


\begin{tabular}{|c|c|}
\hline$\sigma(\lambda)$ & $\begin{array}{l}\text { Standard deviation on } \lambda \text {, measured by } \\
\text { the residual deviations from the fitted } \\
\text { cosine, } \mathrm{cm}\end{array}$ \\
\hline$\sigma\left(B^{2}\right)$ & $\begin{array}{l}\text { Standard deviation on } B^{2} \text { including the } \\
\text { combined deviations on } \lambda \text { and } B_{11} \text {, } \\
10^{-6} \mathrm{~cm}^{-2}\end{array}$ \\
\hline $\mathrm{X}$ & $\begin{array}{l}\text { Distance from center of pile, perpe } \\
\text { cular to tubes, cm }\end{array}$ \\
\hline $\mathrm{Y}$ & $\begin{array}{l}\text { Distance from center of pile, parall } \\
\text { to tubes, } \mathrm{cm}\end{array}$ \\
\hline Z & Distance from source plane, $\mathrm{cm}$ \\
\hline $\mathrm{P}$ & $\begin{array}{l}\text { Distance from bottom of lattice (or } \\
\text { shutter plane) to source plane, } \mathrm{cm}\end{array}$ \\
\hline Q & $\begin{array}{l}\text { Total height of base (distance from } \\
\text { bottom of lattice to floor), } \mathrm{cm}\end{array}$ \\
\hline $\mathrm{a}$ & $\begin{array}{l}\text { Average pile width, } \mathrm{cm} \text {. Layers are } \\
\text { not always exactly equal so an averag } \\
\text { is taken for the whole pile. }\end{array}$ \\
\hline $\mathrm{b}$ & $\begin{array}{l}\text { Pile length, cm. Stock graphite is } \\
\text { used so the length is always } 4 \text { or } 8 \\
\text { feet. Slight variations indicate that } \\
\text { the fill layers between tubes were } \\
\text { perpendicular to the tube layers for } \\
\text { stability so that the average lengths } \\
\text { are slightly different. }\end{array}$ \\
\hline$\Delta$ & $\begin{array}{l}\text { Distance from the center of the last } \\
\text { process tube to the edge of the pile } \\
\text { less half the lattice spacing. This } \\
\text { is a measure of the deviation of the } \\
\text { pile size from an integral number of } \\
\text { lattice cells. }\end{array}$ \\
\hline IR & Inside radius in $\mathrm{cm}$ \\
\hline OR & Outside radius in $\mathrm{cm}$ \\
\hline
\end{tabular}




\section{$-17-$}

HW -69525

DATA SECTION I

LATTICE GEOMETRY TABLES 

TABLE 1B

SOLID RODS: RADII AND AREAS

\begin{tabular}{|c|c|c|c|c|c|c|c|c|c|c|c|c|}
\hline \multirow[b]{2}{*}{ Case } & \multicolumn{6}{|c|}{ Radii $(\mathrm{cm})$} & \multicolumn{4}{|c|}{ Aluminum } & \multicolumn{2}{|c|}{ Coolant } \\
\hline & Uranium & $\begin{array}{l}\text { Can } \\
\text { IR } \\
\end{array}$ & $\begin{array}{l}\mathrm{Can} \\
\mathrm{OR} \\
\end{array}$ & $\begin{array}{c}\text { Process } \\
\text { Tube } \\
\text { IR } \\
\end{array}$ & $\begin{array}{c}\text { Process } \\
\text { Tube } \\
\mathrm{OR} \\
\end{array}$ & $\begin{array}{c}\text { Graphite } \\
\text { IR } \\
\end{array}$ & $\begin{array}{r}\text { Can } \\
\text { Area } \\
\end{array}$ & $\begin{array}{c}\text { Process } \\
\text { Tube } \\
\text { Area } \\
\end{array}$ & $\begin{array}{c}\text { Total A1 } \\
\text { Area } \\
\text { (Including } \\
\text { end caps) } \\
\end{array}$ & $\mathrm{V}-\mathrm{Al} / \mathrm{U}$ & $\begin{array}{c}\text { Annulus } \\
\text { Thickness } \\
\end{array}$ & Area \\
\hline 1 & 1.175 & 1.18 & 1.27 & 1.50 & 1.59 & 1.922 & 0.68 & 0.87 & 1.76 & 0.427 & 0.208 & $1.80^{* * * *}$ \\
\hline 1 & 1.175 & 1.18 & 1.27 & 1.40 & 1.55 & 1.567 & 0.68 & 1.36 & 2.25 & 0.545 & 0.123 & 1.03 \\
\hline 1 & 1.175 & 1.18 & 1.27 & 1.75 & 1.91 & 1.922 & 0.68 & 1.75 & 2.64 & 0.639 & 0.490 & 4.66 \\
\hline 1 & 1.175 & 1.18 & 1.27 & 1.40 & 1.55 & 1.922 & 0.68 & 1.36 & $3.12 \$ \$$ & 0.757 & $0.123 *$ & $1.03 \%$ \\
\hline 1 & 1.175 & 1.18 & 1.27 & 1.40 & 1.55 & 1.922 & 0,68 & 1.36 & 4.005 & 0.968 & $0.123 \%$ & $1.03 \%$ \\
\hline 2 & 1.175 & 1.18 & 1.27 & 1.50 & 1.59 & 1.922 & 0.68 & 0.87 & 1.76 & 0.424 & $0 . .208$ & 1.93 \\
\hline 2 & 1.175 & 1.18 & 1.27 & 1.40 & 1.55 & 1.567 & 0.68 & 1.36 & 2.25 & 0.542 & 0.123 & 1.03 \\
\hline 2 & 1.175 & 1.18 & 1.27 & 1.75 & 1.91 & 1.922 & 0.68 & 1.75 & 2.64 & 0.636 & 0.490 & 4.61 \\
\hline 2 & 1.175 & 1.18 & 1.27 & 2.02 & 2.20 & 2.215 & 0.68 & 2.31 & 3.20 & 0.771 & 0.750 & 7.84 \\
\hline $2 \mathrm{~A}$ & 1.175 & 1.18 & 1.27 & 1.40 & 1.55 & 1.567 & 0.68 & 1.36 & 2.04 & 0.470 & 0.123 & 1.03 \\
\hline 3 & 1.49 & 1.50 & 1.59 & 1.75 & 1.91 & 1.922 & 0.87 & 1.75 & 2.93 & 0.438 & 0.165 & 1.73 \\
\hline 4 & 1.697 & 1.74 & 1.83 & 2.02 & 2.20 & 2.215 & 1.26 & 2.31 & 4.19 & 0.491 & 0.190 & 2.41 \\
\hline 5 & 1.697 & 1.74 & 1.83 & 2.02 & 2.20 & 2.215 & 1.26 & 2.31 & 4.10 & 0.481 & 0.190 & 2.41 \\
\hline 6 & 1.706 & 1.706 & 1.81 & 2.02 & 2.20 & 2.215 & 1.15 & 2.31 & & & 0.213 & 2.57 \\
\hline 7 & 1.706 & 1.706 & 1.81 & 2.02 & 2.20 & 2.215 & 1.15 & 2.31 & & & 0.213 & 2.57 \\
\hline 8 & 1.727 & 1.74 & 1.83 & 2.13 & 2.20 & 2.215 & 1.01 & 0.97 & 2.77 & 0.323 & 0.296 & 3.73 \\
\hline 8 & 1.727 & 1.74 & 1.83 & 2.02 & 2.20 & 2.215 & 1.01 & 2.31 & 4.11 & 0.480 & 0.190 & 2.33 \\
\hline 9 & 2.11 & 2.13 & 2.20 & None & None & 2.215 & 0.97 & None & 0.97 & 0.069 & None & None \\
\hline 9 & 2.11 & 2.13 & 2.20 & 2.534 & 2.70 & 2.714 & 0.952 & 2.73 & 3.68 & 0.264 & 0.334 & 5.06 \\
\hline 10 & 2.11 & 2.13 & 2.20 & None & None & 2.215 & 0.97 & None & 0.97 & 0.069 & None & None \\
\hline 11 & 2.442 & 2.451 & 2.54 & 2.855 & 3.048 & 3.067 & 1.395 & 3.579 & 4.974 & 0.264 & 0.315 & 5.338 \\
\hline $12 \Phi$ & 3.175 & 3.186 & 3.275 & 3.833 & 4.002 & 4.035 & 1.806 & 4.159 & 5.965 & 0.185 & 0.558 & 12.46 \\
\hline & $\begin{aligned} * & \text { Coolar } \\
* & \text { Water } \\
& \text { water } \\
\$ & \text { Includ } \\
\$ \$ & \text { Includ } \\
\mathbb{I} & \text { The ca }\end{aligned}$ & $\begin{array}{l}\text { a secor } \\
\text { a secor } \\
\text { OR is c }\end{array}$ & $\begin{array}{l}\text { proces } \\
\text { proces } \\
\text { rrected }\end{array}$ & $\begin{array}{l}\text { only. } \\
3 \text { and } 7 \\
\text { an accur } \\
\text { be } 1.75 \\
\text { be } 1.50 \\
\text { aluminu }\end{array}$ & $\begin{array}{l}\text { th agree } \\
\text { han the } \\
91 \mathrm{~cm}, \\
69 \mathrm{~cm}, \\
\text { inners in }\end{array}$ & $\begin{array}{l}\text { the alum } \\
\text { inum din } \\
1.75 \mathrm{~cm} \\
0.87 \mathrm{~cm} \\
\text { d of the }\end{array}$ & $n$ di & ions $\mathrm{s}$ & ne value & base & asured & \\
\hline
\end{tabular}



TABLE 2B

CLUSTERED RODS: RADII AND AREAS

\begin{tabular}{|c|c|c|c|c|c|c|c|c|c|c|c|c|}
\hline \multirow[b]{2}{*}{ Case } & \multicolumn{6}{|c|}{ Radii $(\mathrm{cm})$} & \multicolumn{4}{|c|}{ Aluminum } & \multirow[b]{2}{*}{$\begin{array}{l}\text { Coolant } \\
\text { Area } \\
\end{array}$} & \multirow[b]{2}{*}{$\begin{array}{l}\text { Number } \\
\text { of Rods }\end{array}$} \\
\hline & Uranium & $\begin{array}{c}\text { Can } \\
\text { IR }\end{array}$ & $\begin{array}{r}\mathrm{Can} \\
\mathrm{OR} \\
\end{array}$ & $\begin{array}{c}\text { Process } \\
\text { Tube } \\
\text { IR } \\
\end{array}$ & $\begin{array}{c}\text { Process } \\
\text { Tube } \\
\text { OR } \\
\end{array}$ & $\begin{array}{c}\text { Graphite } \\
\text { IR } \\
\end{array}$ & $\begin{array}{c}\text { Can } \\
\text { Area } \\
\end{array}$ & $\begin{array}{c}\text { Process } \\
\text { Tube } \\
\text { Area } \\
\end{array}$ & $\begin{array}{c}\text { Total Al } \\
\text { Area } \\
\text { (Including } \\
\text { end caps) }\end{array}$ & $\mathrm{V}-\mathrm{Al} / \mathrm{U}$ & & \\
\hline 1 & 0.635 & 0.640 & 0.757 & 2.534 & 2.699 & 2.714 & 3.594 & 2.712 & 6.306 & 0.711 & 7.57 & $7 \mathrm{~S}$ \\
\hline 2 & 1.175 & 1.18 & 1.274 & 4.178 & 4.343 & * & 5.08 & 4.42 & 10.97 & 0.379 & 19.16 & $7 \mathrm{~S}$ \\
\hline 3 & 1.175 & 1.18 & 1.274 & 3.835 & 4.001 & $*$ & 5.08 & 4.09 & 10.68 & 0.369 & 10.45 & $7 \mathrm{U}$ \\
\hline 4 & 1.175 & 1.18 & 1.274 & 3.835 & 4.001 & $*$ & 4.35 & 4.09 & 9.74 & 0.394 & 15.61 & $6 \mathrm{U}$ \\
\hline 5 & 1.175 & 1.18 & 1.274 & 3.835 & 4.001 & $*$ & 3.63 & 4.09 & 8.80 & 0.427 & 20.71 & $5 \mathrm{~S}$ \\
\hline
\end{tabular}

The can area, total aluminum area, and A1/U ratio are all calculated using the total number of rods in cluster.

* The graphite holes for the 0.925 -inch clusters were square holes with small pieces of graphite used to make the volds smaller. The equivalent radius of the square hole is listed below for each lattice spacing studied:

$\begin{array}{ll}\text { Separated } 7 \text { Rod } & \begin{array}{c}\text { Unseparated } \\ \text { and }\end{array} \\ 7 \mathrm{in.}-4.806 \mathrm{~cm} & \text { 5Rod Separated } \\ 10-3 / 8 \mathrm{in} .-5.105 \mathrm{~cm} & 7 \mathrm{in.}-4.806 \mathrm{~cm} \\ 14 \mathrm{in} .-4.806 \mathrm{~cm} & 10-3 / 8 \mathrm{in} .-4.953 \mathrm{~cm} \\ & 14 \mathrm{in} .-5.017 \mathrm{~cm}\end{array}$

Polyethylene spacers were used to position the fuel for Cases 1 and 2. The equivalent areas (uniformly distributed) are $0.158 \mathrm{~cm}^{2}$ for Case 1 and $0.50 \mathrm{~cm}^{2}$ for Case 2 . The polyethylene is included with the coolant area. 

TABLE 3B

HOLLOW RODS: RADII AND AREAS

\begin{tabular}{|c|c|c|c|c|c|c|c|c|c|c|c|c|c|c|c|c|c|c|c|c|}
\hline \multirow[b]{2}{*}{ Case } & \multicolumn{9}{|c|}{ Radii $(\mathrm{cm})$} & \multicolumn{5}{|c|}{ Aluminum } & \multicolumn{5}{|c|}{ Coolant } & \multirow[b]{2}{*}{$\begin{array}{r}\text { Coolant } \\
\text { Location } \\
\end{array}$} \\
\hline & $\begin{array}{c}\mathrm{U} \\
\mathrm{OR} \\
\end{array}$ & $\begin{array}{l}\mathrm{U} \\
\mathrm{IR} \\
\end{array}$ & $\begin{array}{l}\text { Outer } \\
\text { Can } \\
\text { IR } \\
\end{array}$ & $\begin{array}{l}\text { Outer } \\
\text { Can } \\
\text { OR } \\
\end{array}$ & $\begin{array}{c}\text { Process } \\
\text { Tube } \\
\text { IR } \\
\end{array}$ & $\begin{array}{l}\text { Process } \\
\text { Tube } \\
\text { OR } \\
\end{array}$ & $\begin{array}{l}\text { Inner } \\
\text { Can } \\
\text { IR } \\
\end{array}$ & $\begin{array}{c}\text { Inner } \\
\text { Can } \\
\text { OR } \\
\end{array}$ & $\begin{array}{c}\text { Graphite } \\
\text { IR } \\
\end{array}$ & $\begin{array}{c}\text { Inner } \\
\text { Can } \\
\text { Area } \\
\end{array}$ & $\begin{array}{l}\text { Outer } \\
\text { Can } \\
\text { Area } \\
\end{array}$ & $\begin{array}{l}\text { Process } \\
\text { Tube } \\
\text { Area } \\
\end{array}$ & $\begin{array}{c}\text { Total } \\
\text { A1 } \\
\text { Area } \\
\end{array}$ & $\underline{\mathrm{V}-\mathrm{A} I / \mathrm{U}}$ & $\begin{array}{r}\text { Core } \\
\text { Radius }\end{array}$ & $\begin{array}{c}\text { Outer } \\
\text { Annulus } \\
\text { Thickness } \\
\end{array}$ & $\begin{array}{r}\text { Area } \\
\text { (core) } \\
\end{array}$ & $\begin{array}{c}\text { Area } \\
\text { (annulus) } \\
\end{array}$ & $\begin{array}{l}\text { Total } \\
\text { Area } \\
\end{array}$ & \\
\hline 1 & 1.727 & 0.635 & 1.74 & 1.83 & 2.02 & 2.20 & None & None & 2.215 & None & 1.01 & 2.31 & 4,11 & 0.554 & None & 0.19 & None & 2.33 & 2.33 & $E$ \\
\hline 2 & 1.740 & 0.610 & 1.74 & 1.87 & 2.13 & 2.20 & 0.48 & 0.61 & 2.215 & 0.446 & 1.473 & 0.97 & 3.66 & 0.473 & 0.48 & 0.26 & 0.72 & 3.16 & 3.88 & $\mathrm{I}$ and $\mathrm{E}$ \\
\hline 3 & 1.740 & 0.610 & 1.74 & 1.87 & 2.13 & 2.20 & 0.48 & 0.61 & 2.215 & 0.446 & 1.473 & 0.97 & 3.66 & 0.473 & 0.48 & 0.26 & 0.72 & 3.16 & 3.88 & $I$ and $E$ \\
\hline 3 & 1.740 & 0.610 & 1.74 & 1.87 & 2.06 & 2.24 & 0.48 & 0.61 & 2.264 & 0.446 & 1.473 & 2.36 & 5.05 & 0.654 & 0.48 & 0.22 & 0.72 & 2.73 & 3.45 & $I$ and $E$ \\
\hline 4 & 2.108 & 1.029 & 2.13 & 2.20 & None & None & 0.87 & 1.02 & 2.215 & 0.89 & 0.97 & None & 1.86 & 0.175 & 0.87 & None & 2.38 & None & 2.38 & I \\
\hline 5 & 2.108 & 1.194 & 2.13 & 2.20 & None & None & 0.87 & 1.02 & 2.215 & 0.89 & 0.97 & None & 1.86 & 0.196 & 0.87 & None & 2.38 & None & 2.38 & I \\
\hline 6 & 2.108 & 1.422 & 2.13 & 2.20 & None & None & 1.26 & 1.40 & 2.215 & 1.18 & 0.97 & None & 2.15 & 0.283 & 1.26 & None & 4.96 & None & 4.96 & I \\
\hline 7 & 3.175 & 2.032 & 3.188 & 3.28 & 3.83 .3 & 4.002 & 1.924 & 2.013 & 4.035 & 1.10 & 1.869 & 4.159 & 7.128 & 0.381 & 1.19 & 0.553 & 11.63 & $12.36 \quad 2$ & 23.99 & $\mathrm{I}$ and $\mathrm{E}$ \\
\hline
\end{tabular}

Cases 2, 3 and 7: The OR of the can is corrected for Al runners instead of the IR of the process tube.

TABLE 3(a)B

SOLID TO HOLLOW FUEL COMPARISON: RADII AND AREAS

\begin{tabular}{|c|c|c|c|c|c|c|c|c|c|c|c|c|c|c|c|c|c|c|c|c|}
\hline \multirow[b]{2}{*}{ Case } & \multicolumn{9}{|c|}{$\operatorname{Radii}(\mathrm{cm})$} & \multicolumn{5}{|c|}{ Aluminum } & \multicolumn{6}{|c|}{ Coolant } \\
\hline & $\begin{array}{l}\mathrm{U} \\
\mathrm{OR} \\
\end{array}$ & $\begin{array}{l}\mathrm{U} \\
\mathrm{IR}\end{array}$ & $\begin{array}{c}\text { Outer } \\
\text { Can } \\
\text { OR } \\
\end{array}$ & $\begin{array}{l}\text { Outer } \\
\text { Can } \\
\text { IR } \\
\end{array}$ & $\begin{array}{c}\text { Process } \\
\text { Tube } \\
\text { OR } \\
\end{array}$ & $\begin{array}{c}\text { Process } \\
\text { Tube } \\
\text { IR } \\
\end{array}$ & $\begin{array}{c}\text { Inner } \\
\text { Can } \\
\mathrm{OR} \\
\end{array}$ & $\begin{array}{l}\text { Inner } \\
\text { Can } \\
\text { IR } \\
\end{array}$ & $\begin{array}{c}\text { Graphite } \\
\text { IR }\end{array}$ & $\begin{array}{l}\overline{\text { Inner }} \\
\text { Can } \\
\text { Area } \\
\end{array}$ & $\begin{array}{l}\text { Outer } \\
\text { Can } \\
\text { Area } \\
\end{array}$ & $\begin{array}{c}\text { Process } \\
\text { Tube } \\
\text { Area } \\
\end{array}$ & $\begin{array}{l}\text { Total } \\
\text { Al } \\
\text { Area } \\
\end{array}$ & $\mathrm{V}-\mathrm{A} 1 / \mathrm{U}$ & $\begin{array}{c}\text { Core } \\
\text { Radius }\end{array}$ & $\begin{array}{c}\text { Outer } \\
\text { Annulus } \\
\text { Thickness } \\
\end{array}$ & $\begin{array}{r}\text { Area } \\
\text { (core) } \\
\end{array}$ & $\begin{array}{c}\text { Area } \\
\text { (annulus) }\end{array}$ & $\begin{array}{l}\text { Total } \\
\text { Area } \\
\end{array}$ & $\begin{array}{r}\text { Coolant } \\
\text { Location } \\
\end{array}$ \\
\hline 1 & 1.722 & 0.526 & 1.854 & 1.722 & 2.20 & 2.02 & 0.52 & 0.39 & 2.215 & 0.382 & 1.48 & 2.31 & 4.76 & 0.605 & 0.394 & 0.166 & 0.487 & 2.02 & 2.51 & $\mathrm{I}$ and $\mathrm{E}$ \\
\hline 2 & 1.697 & $\cdots$ & 1.830 & 1.697 & 2.20 & 2.02 & -- & -- & 2.215 & --- & 1.47 & 2.31 & 4.40 & 0.522 & -.. & 0.190 & -.. & 2.30 & 2.30 & $\mathrm{E}$ \\
\hline 3 & 1.740 & 0.611 & 1.872 & 1.740 & 2.24 & 2.06 & 0.61 & 0.48 & 2.264 & 0.446 & 1.47 & 2.32 & 4.83 & 0.622 & 0.48 & 0.188 & 0.724 & 2.35 & 3.09 & $\mathrm{I}$ and $\mathrm{E}$ \\
\hline 4 & 1.697 & $\ldots$ & 1.830 & 1.697 & 2.24 & 2.06 & -- & -- & 2.264 & --- & 1.47 & 2.32 & 4.41 & 0.523 & $\cdots$ & 0.230 & --- & 2.81 & 2.81 & $E$ \\
\hline
\end{tabular}


TABLE 4A

TUBE AND ROD ELEMENTS URANIUM GEOMETRY

\begin{tabular}{|c|c|c|c|c|c|c|c|c|c|c|c|c|c|c|c|c|}
\hline Case & $\begin{array}{c}\text { Tube } \\
\text { Diameter } \\
\text { (nnches) } \\
\end{array}$ & $\begin{array}{c}\text { Rod } \\
\text { Dameter } \\
\text { (ninches) } \\
\end{array}$ & Enrichment & $\begin{array}{c}\text { Tube } \\
\text { Outside } \\
\text { Radius } \\
\text { (cm) } \\
\end{array}$ & $\begin{array}{l}\text { Tube } \\
\text { Inside } \\
\text { Radust } \\
\text { (cm) }\end{array}$ & $\begin{array}{c}\text { Rod } \\
\text { Radius } \\
\text { (cm) } \\
\end{array}$ & $\begin{array}{l}\text { Tube } \\
\text { Area } \\
\left(\mathrm{cm}^{2}\right)\end{array}$ & $\begin{array}{r}\text { Rod } \\
\text { Area } \\
\left(\mathrm{cm}^{2}\right) \\
\end{array}$ & $\begin{array}{l}\text { Total } \\
\text { Area } \\
\left(\mathrm{cm}^{2}\right) \\
\end{array}$ & $\begin{array}{l}\text { Tube } \\
\text { Length } \\
\text { (cm) }\end{array}$ & $\begin{array}{c}\text { Rod } \\
\text { Length } \\
\text { (cm) } \\
\end{array}$ & $\begin{array}{c}\text { o Tube } \\
\text { (grams } / \mathrm{cm}^{3} \text { ) }\end{array}$ & $\begin{array}{c}\text { Rodd } \\
\left.\text { (grams } / \mathrm{cm}^{3}\right)\end{array}$ & $\begin{array}{c}\text { Paverage } \\
\left.\text { (grams } / \mathrm{cm}^{3}\right)\end{array}$ & $\begin{array}{l}\text { Double } \\
\text { EndCap }\end{array}$ & $\begin{array}{c}\mathrm{S} / \mathrm{M} \\
\mathrm{cm}^{2} \text { (grams) }\end{array}$ \\
\hline 1 & $2.50 \times 1.60$ & 0.50 & N.U. & 3. 175 & 2.032 & 0.635 & 18.70 & 1.266 & 19.97 & 15.24 & 30.48 & 18.78 & 18.65 & 18.76 & None & 0.056 \\
\hline 2 & $2.50 \times 1.60$ & 1.175 & v. v. & 3.175 & 2.032 & 1.492 & 18.70 & 6.975 & 25.68 & 15.24 & 19.37 & 18.78 & 18.95 & 18.82 & None & 0.041 \\
\hline
\end{tabular}

Surface-to-mass ratios $2 n$ these cases are determined from the outside cylinder only.
The masses used are from the tube and the rod together.

TABLE 4B

TUBE AND ROD ELEMENTS RADII AND AREAS

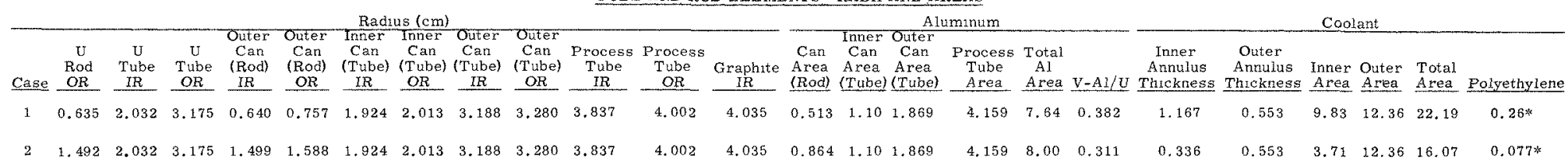

Alumnum runners are nicluded in the outer can instead of the process tube.

* Polyethylene spacers are used to center the fuel and are considered part of the water volume for the wet case. 
TABLE 5A

TUBE IN TUBE ELEMENTS: URANIUM GEOMETRY

Case 1

Inner Tube Diameter (inches)

$1.66 \times 1.12$

Outer Tube Diameter (inches)

$2.50 \times 2.00$

Enrichment

N.U.

Inner Tube IR $(\mathrm{cm})$

1.422

OR $(\mathrm{cm})$

2.108

Outer Tube IR $(\mathrm{cm})$

2.54

OR $(\mathrm{cm})$

3.175

Inner Tube Area $\left(\mathrm{cm}^{2}\right)$

7.609

Outer Tube Area $\left(\mathrm{cm}^{2}\right)$

11.401

Total Area $\left(\mathrm{cm}^{2}\right)$

19.01

Inner Tube Length $(\mathrm{cm})$

10.16

Outer Tube Length $(\mathrm{cm})$

20.32

Inner Tube $\rho$ (grams $/ \mathrm{cm}^{3}$ )

18.60

Outer Tube $p\left(\right.$ grams $\left./ \mathrm{cm}^{3}\right)$

18.95

p Average (grams $/ \mathrm{cm}^{3}$ )

18.81

Double End Cap

None

$\mathrm{S} / \mathrm{M}\left(\mathrm{cm}^{2} /\right.$ grams $)$

0.0555

Surface-to-mass ratio was determined from the outer surface of the outer tube and the masses of both tubes. 
TABLE 5B

TUBE IN TUBE ELEMENTS: RADII AND AREAS

$\underline{\operatorname{Radii}(\mathrm{cm})}$

Uranium Inner Tube, IR 1.422 OR 2.108

Uranium Outer Tube, IR

2.54

OR 3.175

Inner Tube, Inner Can, IR 1.257

OR 1.397

Inner Tube, Outer Can, IR 2.13

OR 2.20

Outer Tube, Inner Can, IR 2.438

OR 2.527

Outer Tube, Outer Can, IR 3.188

OR (corrected for runners) $\quad 3.280$

Process Tube, IR 3.837

$\begin{array}{ll}\text { OR } & 4.002\end{array}$

Graphite, IR $\quad 4.035$

Aluminum

Inner Tube, Inner Can, Area 1.168

Inner Tube, Outer Can, Area $\quad 0.952$

Outer Tube, Inner Can, Area 1.388

Outer Tube, Outer Can, Area 1.869

Process Tube Area $\quad 4.159$

Total Aluminum Area $\quad 9.536$

$\mathrm{V}-\mathrm{Al} / \mathrm{U} \quad 0.502$

Coolant

Core Radius $(\mathrm{cm}) \quad 1.257$

Inner Annulus Thickness (cm) 0.238

Outer Annulus Thickness $(\mathrm{cm}) \quad 0.553$

Area (core) 4.96

$\begin{array}{lr}\text { (inner annulus) } & 3.47\end{array}$

(outer annulus) 12.36

Total Area

Polyethylene

20.79

$0.072 *$

* Polyethylene spacers used to center the fuel. Spacers are considered part of the water volume for wet case. 
TABLE 6

EQUIVALENT CELL RADII

FOR ALL LATTICE SPACINGS

\begin{tabular}{c}
$\begin{array}{c}\text { Lattice Spacing } \\
\text { (inches) }\end{array}$ \\
\hline $4-3 / 16$ \\
$5-3 / 16$ \\
$6-3 / 16$ \\
7 \\
$7-3 / 16$ \\
$7-1 / 2$ \\
$7-11 / 16$ \\
8 \\
$8-3 / 8$ \\
$9-3 / 8$ \\
$10-3 / 8$ \\
$12-3 / 8$ \\
14 \\
$14-9 / 16$ \\
15 \\
$4-3 / 16 \times 8-3 / 8$
\end{tabular}

Equivalent

Cell Radius (cm)

6.001

7. 434

8.867

10.031

10.300

10.748

11.016

11. 464

12.001

13. 434

14.868

17.734

20.063

20.876

21. 496

8.486 
DATA SECTION II

MATERIAL BUCKLING TABLES 
TABLE 1

CASE 1. 0.925-INCH RODS, N.U.

\section{a. 8-Foot Pile}

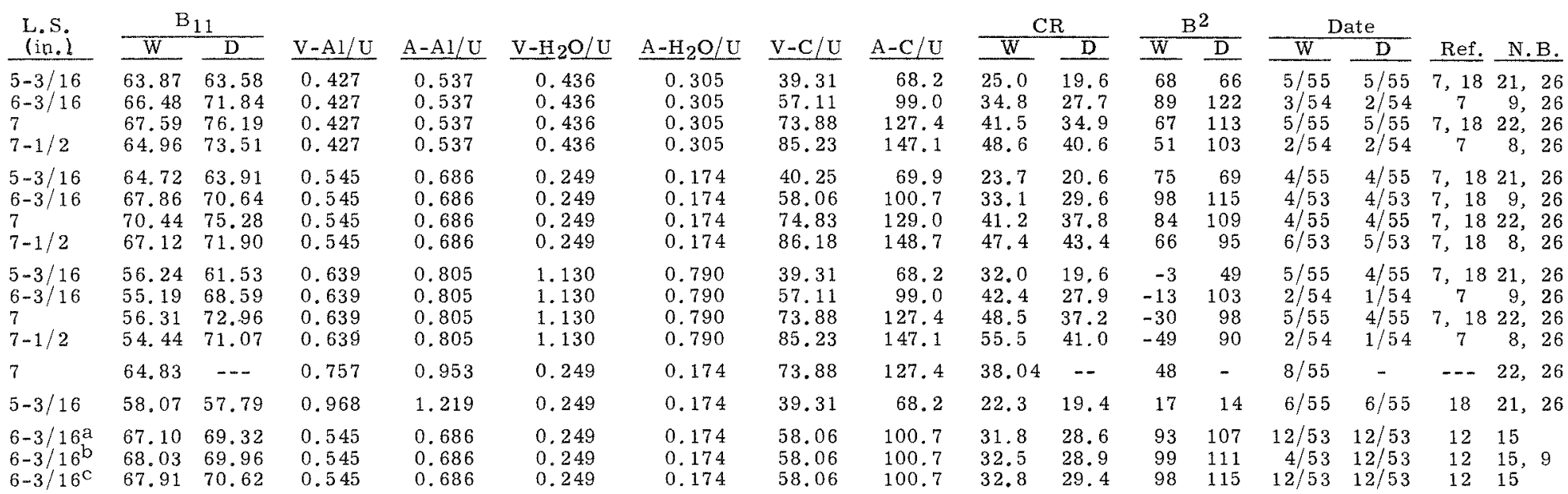

a, b, c: Core loadings of 0.925 -inch rods surrounded by 1.17 -inch rods to study precision of core measurements. The numbers of tubes of $0.925 m$ inch fuel for $a, b$, and $c$, are $4 \times 7,6 \times 7$, and $8 \times 7$, respectively.

\section{TABLE 1}

CASE 1. $0.925-\mathrm{INCH}$ RODS, N. U. *

\section{b. 4-Foot Pile}

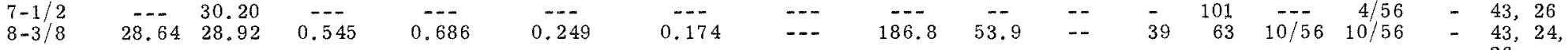

* Clustered sources were used and the extrapolation length was assumed to be 1.15 inches. 
TABLE 1

CASE 2. $0.925-$ INCH RODS, 1.007 PER CENT ENRICHMENT

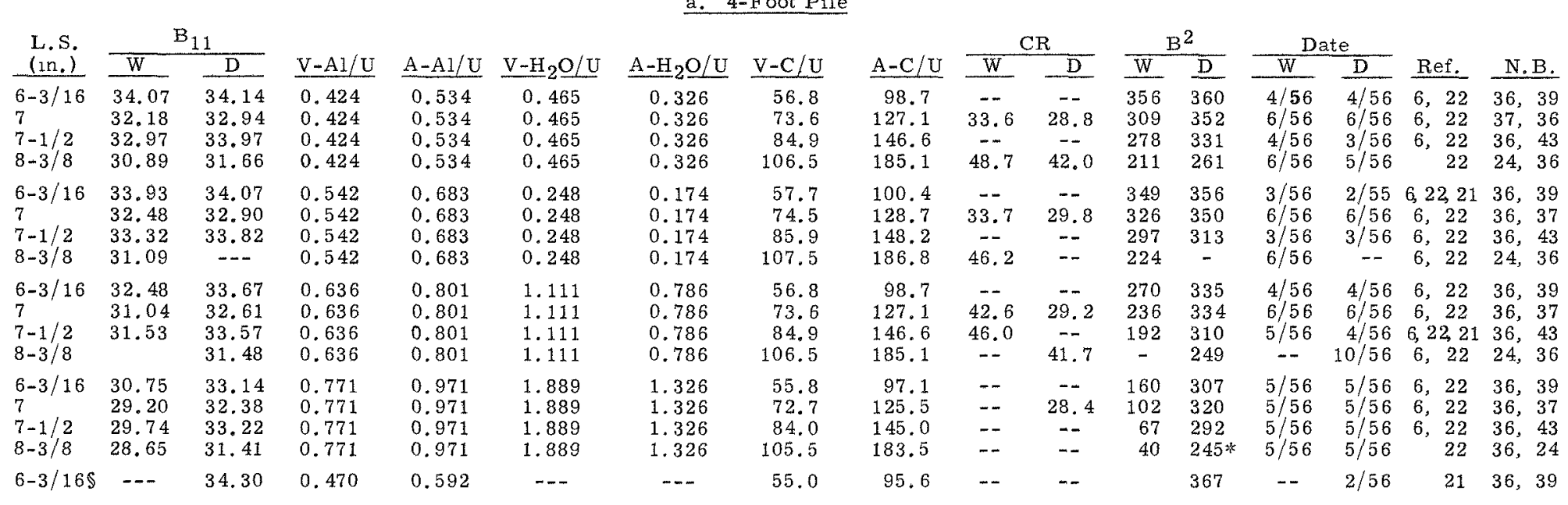

Clustered sources were used and the extrapolation distance was assumed to be 1.15 inches.

* A second measurement in October, 1956, gave $\mathrm{B}^{2}=249$.

$\$$ Case 2A: no spacers between fuel. 
TABLE 1

CASE 3. 1.17-INCH RODS, N.U.

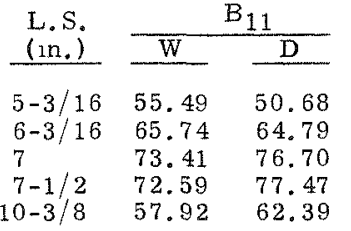

$\begin{array}{lll}10-3 / 8 & 57.92 & 62.39\end{array}$

\begin{tabular}{|c|c|c|}
\hline$-\mathrm{A} 1 / \mathrm{U}$ & $\mathrm{A}-\mathrm{A} 1 / \mathrm{U}$ & $\underline{\mathrm{V}-\mathrm{H}_{2}} 2 \mathrm{O} / \mathrm{U}$ \\
\hline 0.43 & 0.551 & 0.258 \\
\hline 0.438 & 0.551 & 0.258 \\
\hline 0.438 & 0.551 & 0.258 \\
\hline 0.438 & 0.551 & 0.258 \\
\hline 0.438 & 0.551 & 0.258 \\
\hline
\end{tabular}

\section{a. 8-Foot Ple}

\begin{tabular}{|c|c|c|c|c|c|c|c|c|c|c|}
\hline \multirow[b]{2}{*}{$\mathrm{A}-\mathrm{H}_{2} \mathrm{O} / \mathrm{U}$} & \multirow[b]{2}{*}{$\mathrm{V}-\mathrm{C} / \mathrm{U}$} & \multirow[b]{2}{*}{$\underline{A-C} / \mathrm{U}$} & \multicolumn{2}{|c|}{$\mathrm{CR}$} & \multicolumn{2}{|c|}{$\mathrm{B}^{2}$} & \multicolumn{2}{|c|}{ Date } & \multirow[b]{2}{*}{ Ref. } & \multirow[b]{2}{*}{ N.B. } \\
\hline & & & $\mathrm{W}$ & $\bar{D}$ & $\mathrm{~W}$ & D & W & $\mathrm{D}$ & & \\
\hline 0.18 & 23. & 41. & 17.6 & 14.1 & -11 & -76 & $6 / 55$ & $6 / 55$ & 7,19 & 21,30 \\
\hline 0 & & & 24. & & 84 & 77 & $12 / 55$ & & & 12 \\
\hline & 45. & 78 & 29.8 & 26.2 & 100 & 116 & $7 / 55$ & $8 / 55$ & 7,19 & 22, \\
\hline 0. & 52. & 90 & 34.8 & 28. & 99 & 122 & $11 / 53$ & $11 / 53$ & 7,19 & 14,30 \\
\hline 0.180 & 102.76 & 178.7 & 60.5 & 55.0 & 15 & 57 & $7 / 55$ & $7 / 55$ & 7,19 & 20,30 \\
\hline
\end{tabular}

TABLE 1

CASE 4. 1.336-INCH RODS, N.U. a. 4-Foot Pile

$8-3 / 8 \quad 34.00 \quad 34.96 \quad 0.491$

0.618

0.286

0.200

52.15

90.1

$38.2 \quad 32.0$

$\begin{array}{lllll}79 & 126 & 3 / 57 & 3 / 57 & -\end{array}$

44

TABLE 1

CASE 5. 1.336-INCH RODS, 0.94 PER CENT ENRICHMENT

$\begin{array}{cccc}5-3 / 16 & 27.18 & 26.10 & 0.481 \\ 6-3 / 16 & 30.12 & 29.83 & 0.481 \\ 7-3 / 16 & 33.75 & 33.67 & 0.481 \\ 8-3 / 8 & 32.32 & 32.63 & 0.481 \\ 10-3 / 8 & 30.61 & 31.43 & 0.481\end{array}$

\begin{tabular}{|c|c|c|c|c|c|c|c|c|c|c|c|c|}
\hline 0.61 & 0.283 & 0.198 & 18.57 & 32.3 & 13.6 & 9.7 & 226 & 112 & $11 / 56$ & $11 / 56$ & 6,24 & 42,34 \\
\hline & & 0.198 & 27.15 & 47.2 & 19. & 14.6 & 30 & 286 & $8 / 56$ & $7 / 56$ & & 39 , \\
\hline 6. & 0.283 & 0.198 & 37.21 & 64.7 & 25.0 & 2.0 .4 & 326 & 321 & $9 / 56$ & $9 / 56$ & & 33, \\
\hline 61 & 0.283 & 0.198 & 51.59 & 89.1 & 33. & 28.2 & 301 & 320 & $8 / 56$ & $7 / 56$ & & \\
\hline 0.61 & 0.283 & 0.198 & 80.54 & 138.5 & 47.4 & 41.6 & 201 & 256 & $12 / 56$ & $12 / 56$ & 6,24 & 32, \\
\hline
\end{tabular}

TABLE 1

CASE 6. 1.343-INCH RODS, 4.46 PER CENT ENRICHED U-AI ALLOY

a. 4-Foot Pile

\begin{tabular}{|c|c|c|c|c|c|c|c|c|c|c|c|c|c|c|c|c|}
\hline $4-3 / 16$ & 66.49 & 41.69 & $-\cdots$ & -- & $\cdots$ & -- & $\cdots$ & -- & 13.1 & 8.5 & 1572 & 1222 & $12 / 55$ & $12 / 55$ & 6 & 35,45 \\
\hline $5-3 / 16$ & 52.42 & 110.82 & $\cdots$ & -- & -- & -- & $\cdots$ & -- & 19.0 & 131 & 1323 & 1134 & $12 / 55$ & $12 / 55$ & 6 & 35,45 \\
\hline $6-3 / 16$ & 84.5 & 69.46 &.- & -- & ... & $\cdots$ & $\ldots$ & -- & 23.2 & 18.0 & 1078 & 1010 & $11 / 55$ & $12 / 55$ & 6 & 39,45 \\
\hline $7-3 / 16$ & 55.82 & 54.10 & -- & -- & $\ldots$ & $\ldots$ & -- & -- & 29.3 & 24.5 & 897 & 877 & $10 / 55$ & $10 / 55$ & 6 & 23,45 \\
\hline $8-3 / 8$ & 44.77 & 46.04 & $\cdots$ & - & $-m$ & $\cdots$ & $\cdots$ & -- & 37.7 & 33.3 & 709 & 736 & $9 / 55$ & $9 / 55$ & 6 & 35,45 \\
\hline $10-3 / 8$ & 36.63 & 37.92 &.- & -- & $\ldots$ & $\ldots$ & $-\cdots$ & -- & 52.4 & 37.1 & 470 & 519 & $11 / 55$ & $11 / 55$ & 6 & 35,45 \\
\hline
\end{tabular}




\section{TABLE 1}

CASE 7. 1.343-INCH RODS, 7.5 PER CENT ENRICHED U-Al ALLOY

a. 4-Foot Pile

\begin{tabular}{|c|c|c|c|c|c|c|c|c|}
\hline \multirow{2}{*}{$\begin{array}{l}\text { L.S. } \\
\text { (in.) }\end{array}$} & \multicolumn{2}{|c|}{$\mathrm{B}_{11}$} & \multicolumn{2}{|c|}{$\mathrm{CR}$} & \multicolumn{2}{|c|}{$B^{2}$} & \multicolumn{2}{|c|}{ Date } \\
\hline & W & $\bar{D}$ & W & $\mathrm{D}$ & $\bar{W}$ & $\mathrm{D}$ & W & $\mathrm{D}$ \\
\hline $4-3 / 16$ & 63.37 & 51.35 & -- & -- & 1886 & 1419 & $1 / 56$ & $1 / 56$ \\
\hline $5-3 / 16$ & 116.08 & 56.69 & -- & 10.3 & 1612 & 1375 & $1 / 56$ & $1 / 56$ \\
\hline $6-3 / 16$ & -- & $-\cdots$ & 18.8 & 14.1 & 1368 & 1258 & $6 / 55$ & $6 / 55$ \\
\hline $7-3 / 16$ & 134.79 & 102.3 & 23.4 & 18.6 & 1163 & 1122 & $7 / 55$ & $7 / 55$ \\
\hline $8-3 / 8$ & 64.23 & 65.06 & 30.4 & 25.7 & 965 & 972 & $9 / 55$ & $9 / 55$ \\
\hline $10-3 / 8$ & 43.28 & 45.07 & 41.1 & 36.4 & 678 & 719 & $8 / 55$ & $8 / 55$ \\
\hline $\begin{array}{l}4-3 / 16 \\
\times 8-3 / 8 \$\end{array}$ & $\ldots$ & 89.48 & 15.3 & 12.4 & 1166 & 1041 & $5 / 54$ & $5 / 54$ \\
\hline $7-3 / 16^{*}$ & -- & 97.68 & -- & 19.0 & -- & 1114 & -- & $6 / 55$ \\
\hline & $\begin{array}{l}* \text { Uppe } \\
\$ \text { Clust } \\
\text { Thre } \\
(0, \pm\end{array}$ & $\begin{array}{l}\text { ource } \\
\text { ed sour } \\
\text { ources } \\
4 \text { 4) }\end{array}$ & $\begin{array}{l}\text { on. } \\
\text { yere }\end{array}$ & $\begin{array}{l}\text { other } \\
d \text { and } \\
r \text { all }\end{array}$ & $\begin{array}{l}\text { sed } \\
\text { ras } \\
\text { res, }\end{array}$ & ume & $\begin{array}{l}\text { pos } \\
1 . \\
\text { and }\end{array}$ & n. \\
\hline
\end{tabular}


TABLE 1

CASE 8, 1.36-INCH RODS, N.U.

a. 8-Foot Pile

\begin{tabular}{|c|c|c|c|c|c|}
\hline \multirow{2}{*}{$\begin{array}{l}\text { L.S. } \\
\text { (1n.) } \\
\end{array}$} & \multicolumn{2}{|c|}{$B_{11}$} & \multirow[b]{2}{*}{$\mathrm{V}-\mathrm{A} 1 / \mathrm{U}$} & \multirow[b]{2}{*}{$\mathrm{A}-\mathrm{Al} / \mathrm{U}$} & \multirow[b]{2}{*}{$\underline{\mathrm{V}-\mathrm{H}_{2}} 2 \mathrm{O} / \mathrm{U}$} \\
\hline & W & $\mathrm{D}$ & & & \\
\hline $6-3 / 16$ & 62.13 & 58.31 & 0.323 & 0.410 & 0.436 \\
\hline $7-1 / 2$ & 69.99 & 76.33 & 0.323 & 0.410 & 0.436 \\
\hline $12-3 / 8$ & 51.88 & 57.39 & 0.323 & 0.410 & 0.436 \\
\hline $5-3 / 16$ & 48.35 & 43.08 & 0.480 & 0.609 & 0.272 \\
\hline $6-3 / 16$ & 59.22 & 56.48 & 0.480 & 0.609 & 0.272 \\
\hline & 69.49 & 69.85 & 0.480 & 0.609 & 0.272 \\
\hline $7-1 / 2$ & 70.22 & 72.98 & 0.480 & 0.609 & 0.272 \\
\hline & 68.53 & 73.88 & 0.480 & 0.609 & 0.272 \\
\hline $8-3 / 8$ & 65.31 & 70.66 & 0.480 & 0.609 & 0.272 \\
\hline $10-3 / 8$ & & 64.24 & 0.480 & 0.609 & 0.272 \\
\hline $5-3 / 16$ & 49.35 & 45.00 & No data & vailable. & $\ldots$ \\
\hline
\end{tabular}

${\underline{A}-\mathrm{H}_{2}}_{2} \mathrm{O} / \mathrm{U} \quad \mathrm{V}-\mathrm{C} / \mathrm{U} \quad \underline{\mathrm{A}-\mathrm{C} / \mathrm{U}}$

$\frac{\mathrm{CR}}{\mathrm{W}} \frac{\mathrm{B}^{2}}{\mathrm{D}} \stackrel{\mathrm{D}}{\mathrm{D}} \mathrm{W} \stackrel{\mathrm{D}}{\mathrm{W}} \mathrm{Ref}$ N.B.

$\begin{array}{ll}0.305 & 26.99 \\ 0.305 & 40.50\end{array}$

46.9

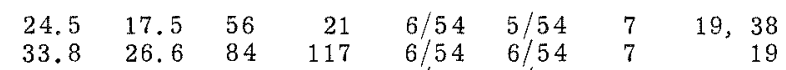

$0.305 \quad 113.30 \quad 196.9$ $\begin{array}{rrrrrr}82.8 & 69.8 & -56 & 12 & 6 / 54 & 5 / 54\end{array}$

0.191

18.44
26.99

32.1
46.9

$\begin{array}{rrrrrr}15.3 & 11.8 & -114 & -225 & 3 / 55 & 1 / 55 \\ 21.5 & 17.2 & 31 & 2 & 3 / 53 & 10 / 52\end{array}$

$0.191 \quad 35.05 \quad 60.5$

$\begin{array}{lll}0.191 & 40.50 & 69.9 \\ 0.191 & 46.32 & 80.0\end{array}$

$\begin{array}{lll}0.191 & 46.32 & 80.0 \\ 0.191 & 50.94 & 88.6 \\ 0.191 & 79.14 & 137.6\end{array}$

$0.191 \quad 79.14 \quad 137.6$

$\begin{array}{rrrrrr}27.3 & 22.3 & 78 & 81 & 2 / 55 & 1 / 55 \\ 31.4 & 20.2 & 85 & 101 & 8 / 52 & 8 / 52\end{array}$

$\begin{array}{llllll}35.0 & 29.6 & 84 & 113 & 7 / 51 & 5 / 51\end{array}$

$\begin{array}{llllll}38.2 & 33.0 & 84 & 118 & 11 / 50 & 11 / 50\end{array}$

$\begin{array}{lllll}48.8 & 26 & 71 & 10 / 54 & 12 / 54\end{array}$

$\begin{array}{llllll}16.7 & 12.4 & -97 & -180 & 3 / 55 & 4 / 55\end{array}$

19,38
19
19,38

19,38
21,38

22,38

38
38

38
38
38

20

$---$

-... -.

TABLE 1

CASE 8. 1.36-INCH RODS, N. U.*

\begin{tabular}{|c|c|c|c|c|c|c|c|c|c|c|c|c|}
\hline \multicolumn{13}{|c|}{ a. 4-Foot Pile } \\
\hline $6-3 / 16$ & 28.64 & 0.480 & 0.609 & ... & -.. & 26.99 & 46.9 & -+ & -2 & $3 / 56$ & 12,11 & 38,39 \\
\hline $7-1 / 2$ & 30.25 & 0.480 & 0.609 & $\ldots$ & ..- & 40.50 & 69.9 & 26.6 & 105 & $3 / 56$ & 11 & 43,38 \\
\hline $8-3 / 8$ & 30.89 & 0.480 & 0.609 & $\ldots$ & ... & 50.94 & 88.6 & 31.3 & 117 & $4 / 54$ & 11 & 16,38 \\
\hline $\begin{array}{c}0-3 / 8 \\
10-3 / 8\end{array}$ & 29.62 & 0.480 & 0.609 & $\ldots$ & $\ldots$ & 79.14 & 137.6 & 45.5 & 74 & $7 / 55$ & 11 & $38, \quad 32$ \\
\hline
\end{tabular}

* Clustered sources were used and the extrapolation distance was assumed to be 1.15 inches.

except for the $8-3 / 8$-inch lattice where $\lambda$ was assumed to be 1.6 inches. 
TABLE 1

CASE 9. 1.66-INCH RODS, N.U.

\begin{tabular}{|c|c|c|c|c|c|c|c|c|c|c|c|c|}
\hline $\begin{array}{l}\text { L.S. } \\
\text { (1n.) }\end{array}$ & $\mathrm{B}_{11}$ & $\mathrm{~V}-\mathrm{Al} / \mathrm{U}$ & $\mathrm{A}-\mathrm{A} \mathrm{l} / \mathrm{U}$ & $\mathrm{V}-\mathrm{H}_{2} \mathrm{O} / \mathrm{U}$ & $\mathrm{A}-\mathrm{H}_{2} \mathrm{O} / \mathrm{U}$ & $\mathrm{V}-\mathrm{C} / \mathrm{U}$ & $\underline{A-C} / U$ & $\frac{\mathrm{CR}}{\mathrm{D}}$ & $\frac{\mathrm{B}^{2}}{\mathrm{D}}$ & $\frac{\text { Date }}{\text { D }}$ & Ref. & N.B. \\
\hline $6-3 / 16$ & 46.26 & 0.0695 & 0.0882 & $\ldots$ & $\ldots$ & 16.59 & 29.0 & 12.6 & -153 & $6 / 53$ & 7,26 & 10,27 \\
\hline $7-1 / 2$ & 68.15 & 0.0695 & 0.0882 & $\ldots$ & -- & 24.85 & 43.3 & 19.3 & 73 & $6 / 53$ & 7,26 & 11,27 \\
\hline $10-3 / 8$ & 71.80 & 0.0695 & 0.0882 & $\ldots$ & ..- & 48.55 & 85.2 & 39.4 & 120 & $8 / 54$ & 26 & 20,27 \\
\hline $12-3 / 8$ & 64.63 & 0.0695 & 0.0882 & --- & -- & 69.54 & 121.9 & 53.8 & 76 & $8 / 53$ & 7,26 & \\
\hline & 58.46 & 0.0695 & 0.0882 & $\ldots$ & $\ldots$ & 102.69 & 179.0 & 76.2 & -4 & $8 / 53$ & 7,26 & 11 \\
\hline
\end{tabular}

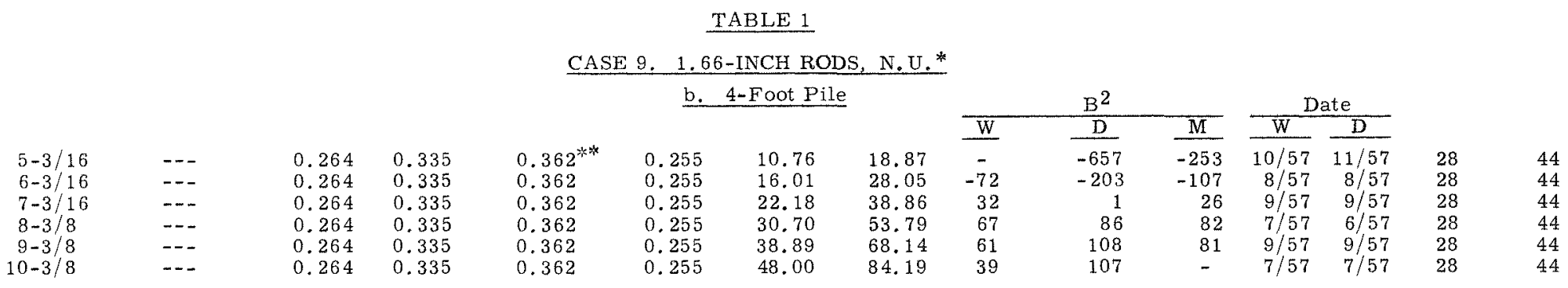

* Clustered sources were used and the extrapolation distance was assumed to be 1.66 inches.

TABLE 1

CASE 10. 1.66-INCH RODS, 1.007 PER CENT ENRICH MENT*

\begin{tabular}{|c|c|c|c|c|c|c|c|c|c|c|c|c|c|c|c|}
\hline & \multicolumn{2}{|c|}{$B_{11}$} & & & & & & & \multicolumn{2}{|c|}{$\mathrm{CR}$} & \multicolumn{2}{|c|}{$B^{2}$} & & & \\
\hline & W & D & & & & & & & $\mathrm{W}$ & D & W & D & & & \\
\hline $5-3 / 16$ & $\ldots$ & 23.33 & 0.069 & 0.087 &.- & -.. & 11.31 & 19.8 & - & -- & -.. & -157 & $2 / 56$ & 6,21 & $42, \quad 29$ \\
\hline $6-3 / 16$ & -- & 30.67 & 0.069 & 0.087 & $\ldots$ & $\ldots$ & 16.56 & 29.0 & - & -- & $\ldots$ & 154 & $2 / 56$ & 6,21 & 39,29 \\
\hline 7 & -- & 32.45 & 0.069 & 0.087 & $\ldots$ &.-- & 21.50 & 37.5 & - & -- &.- & 325 & $2 / 56$ & 6,21 & 37,29 \\
\hline $7-1 / 2$ & $\ldots$ & 34.70 & 0.069 & 0.087 &.-- & $\ldots$ & 24.85 & 43.4 & - & 16.2 & --- & 367 & $2 / 56$ & 6,21 & $42,43,29$ \\
\hline $8-3 / 8$ &.-- & 34.23 & 0.069 & 0.087 & $\ldots$ & $\ldots$ & 31.25 & 54.9 & - & - & -- & 405 & $2 / 56$ & 6,21 & 24,29 \\
\hline $10-3 / 8$ & --- & $\begin{array}{l}33.36 \\
33.36\end{array}$ & 0.069 & 0.087 &.-- & -.- & 48.55 & 85.2 & - & 31.2 &.- & 368 & $2 / 56$ & 6,21 & 29,32 \\
\hline $12-3 / 8$ & --- & 32.46 & 0.069 & 0.087 & $\ldots$ & -.. & 69.54 & 121.9 & - & 45.5 & $-\ldots$ & 268 & $2 / 56$ & 6,21 & 29,44 \\
\hline
\end{tabular}

* Clustered sources were used and the extrapolation distance was assumed to be 1.15 inches. 
TABLE 1

CASE 11. 1.92-INCH RODS, N.U.**

\begin{tabular}{|c|c|c|c|c|c|c|c|c|c|c|c|c|}
\hline \multirow{3}{*}{$\begin{array}{l}\text { L.S. } \\
\text { (in.) }\end{array}$} & \multirow{2}{*}{\multicolumn{2}{|c|}{$\mathrm{B}_{11}$}} & \multirow[b]{3}{*}{$\mathrm{V}-\mathrm{Al} / \mathrm{U}$} & \multirow[b]{3}{*}{$\mathrm{A}-\mathrm{Al} / \mathrm{U}$} & \multirow[b]{3}{*}{$\mathrm{V}-\mathrm{H}_{2} \mathrm{O} / \mathrm{U}$} & & \multirow[b]{3}{*}{$\mathrm{A}-\mathrm{C} / \mathrm{U}$} & \multirow{2}{*}{\multicolumn{2}{|c|}{$\mathrm{CR}$}} & \multirow{2}{*}{\multicolumn{2}{|c|}{$B^{2}$}} \\
\hline & & & & & & & & & & & & \\
\hline & W & D & & & & $\underline{\mathrm{A}-\mathrm{H}_{2}} 2 \mathrm{O} / \mathrm{U}$ & $\mathrm{V}-\mathrm{C} / \mathrm{U}$ & & $\mathrm{W}$ & D & $\mathrm{W}$ & D \\
\hline $3 / 16$ & .72 & .6 & 0 & 0 & 0 . & 0 & 11 & & 14.2 & 8. & $\begin{array}{r}-186 \\
65\end{array}$ & -400 \\
\hline & & & & & & & & & 24 & 18 & 65 & 67 \\
\hline-3 & 33.78 & 34. & & & & & & & 36.6 & & 70 & 127 \\
\hline $2-3 / 8$ & 34.88 & 36.43 & 0.264 & 0.332 & 0.288 & 0.201 & 51.31 & 89.28 & 49.1 & 40.9 & 34 & 102 \\
\hline
\end{tabular}

\begin{tabular}{|c|c|c|c|c|c|c|c|c|c|c|c|c|c|c|c|c|}
\hline & \multicolumn{2}{|c|}{$\mathrm{N}_{\mathrm{B}}$} & \multicolumn{2}{|c|}{$L_{\lambda}$} & \multicolumn{2}{|c|}{$\mathrm{N}_{\lambda}$} & \multicolumn{2}{|c|}{$\lambda_{1}$} & \multicolumn{2}{|c|}{$\lambda_{11}{ }^{*}$} & \multirow[b]{2}{*}{$\sigma(\lambda)^{*}$} & \multicolumn{2}{|c|}{$\sigma\left(\mathrm{B}^{2}\right)$} & \multicolumn{2}{|c|}{ Date } & \multirow[b]{2}{*}{ Ref } \\
\hline & $\underline{W}$ & $\underline{D}$ & $\mathrm{~W}$ & D & $\bar{W}$ & $\overline{\mathrm{D}}$ & W & $\mathrm{D}$ & $\mathrm{W}$ & D & & $\bar{W}$ & $\overrightarrow{\mathrm{D}}$ & W & D & \\
\hline $6-3 / 16$ & 6 & 6 & 4 & 4 & 4 & 4 & 1.34 & 1.20 & 1.03 & 1.03 & 0.25 & 13 & 15 & $4 / 59$ & $4 / 59$ & 35 \\
\hline $8-3 / 8$ & 6 & 6 & 4 & 4 & 4 & 4 & 1.37 & 1.39 & 1.06 & 1.06 & 0.25 & 6 & 6 & $11 / 58$ & $12 / 58$ & 35 \\
\hline $10-3 / 8$ & 6 & 6 & $1+2$ & $1+2$ & 11 & 11 & 1.56 & 1.50 & 1.03 & 1.03 & 0.25 & 5 & 5 & $5 / 59$ & $6 / 59$ & 35 \\
\hline $12-3 / 8$ & 6 & 6 & 3 & 3 & 5 & 5 & 2.11 & 2.25 & 1.03 & 1.03 & 0.25 & 6 & 4 & $4 / 59$ & $4 / 59$ & 35 \\
\hline
\end{tabular}

* Assumed values based on later measurements.

TABLE 1

CASE 12. 2.5-INCH RODS, N.U. $\$ S$

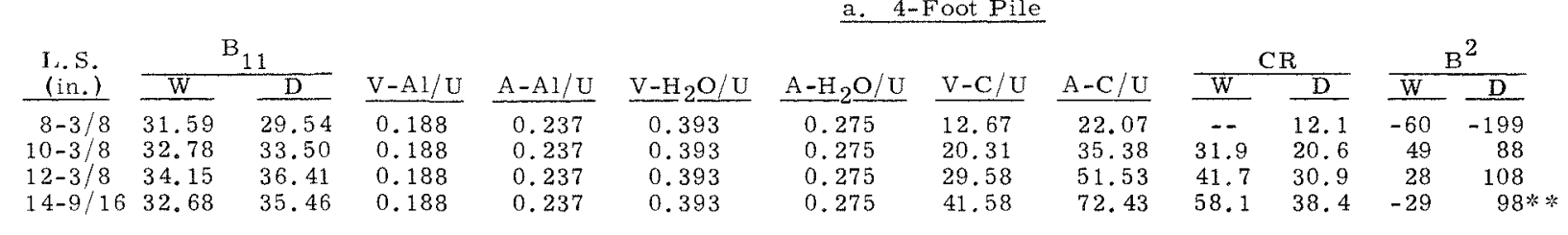

\begin{tabular}{ccc} 
& \multicolumn{2}{c}{$N_{\mathrm{B}}$} \\
\cline { 2 - 2 } & $\frac{\mathrm{W}}{5}$ & D \\
$10-3 / 8$ & 5 & \\
$12-3 / 8$ & 5 & \\
$14-9 / 16$ & 5 & \\
& 4 &
\end{tabular}

\begin{tabular}{cll}
\multicolumn{2}{c}{$L_{\lambda}$} & \\
\hline$\frac{W}{1+2}$ & & $\frac{D}{4}$ \\
$1 \$$ & & $1 \$$ \\
1 & 1 \\
3 & 3
\end{tabular}

\begin{tabular}{lr}
\multicolumn{2}{c}{$\mathrm{N}_{\lambda}$} \\
\hline $\mathrm{W}$ & D \\
8 & 4 \\
3 & 3 \\
3 & 3 \\
5 & 5
\end{tabular}

\begin{tabular}{|c|c|}
\hline \multicolumn{2}{|c|}{$\lambda_{\perp}$} \\
\hline $\mathrm{W}$ & D \\
\hline $\begin{array}{l}1.6 \\
1.4\end{array}$ & 1.6 \\
\hline 2.0 & 1.7 \\
\hline
\end{tabular}

$\frac{\lambda_{11} *}{1.03}$
1.03
1.03
1.03

* Assumed values based on later measurements

**: Average of two experiments

\$ Taken 1 -inch above position 1.

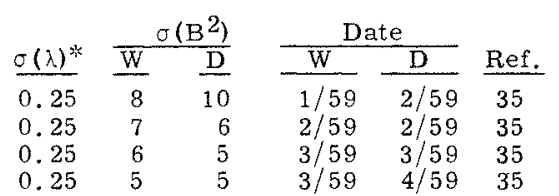


TABLE 2

CASE 1. 0.50-INCH 7-ROD CLUSTERS, N.U.

\begin{tabular}{c}
$\begin{array}{c}\text { L.S. } \\
\text { (in.) }\end{array}$ \\
\hline $5-3 / 16$ \\
$6-3 / 16$ \\
$7-3 / 16$ \\
$8-3 / 8$ \\
$10-3 / 8$
\end{tabular}

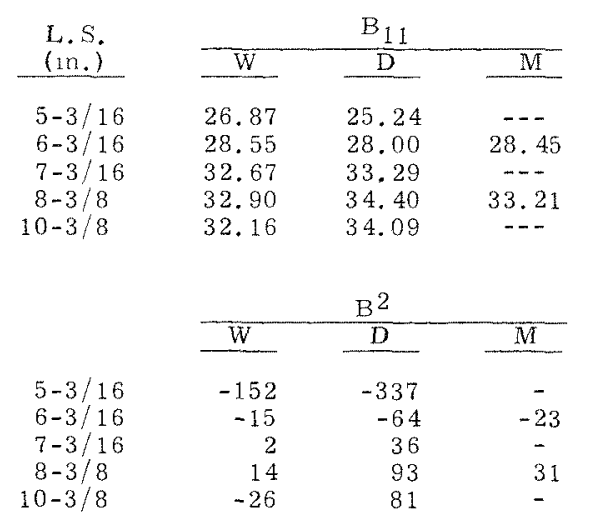

a. 4-Foót Pile

\begin{tabular}{|c|c|c|c|c|c|c|c|c|}
\hline \multirow[b]{2}{*}{$\mathrm{V}-\mathrm{Al} / \mathrm{U}$} & \multirow[b]{2}{*}{$A-A 1 / U$} & \multirow[b]{2}{*}{$\mathrm{V}-\mathrm{H}_{2} \mathrm{O} / \mathrm{U}^{*}$} & \multirow[b]{2}{*}{$\mathrm{A}-\mathrm{H}_{2} \mathrm{O} / \mathrm{U}$} & \multirow[b]{2}{*}{$\mathrm{V}-\mathrm{C} / \mathrm{U}$} & \multirow[b]{2}{*}{$A-C / U$} & \multicolumn{3}{|c|}{$\mathrm{CR}$} \\
\hline & & & & & & W & D & M \\
\hline 0.711 & 0.897 & 0.864 & 0.605 & 16.97 & 29.5 & 18.2 & 9.9 & -- \\
\hline 0.711 & 0.897 & 0.864 & 0.605 & 25.24 & 43.9 & 22.0 & 13.8 & 20.4 \\
\hline 0.711 & 0.897 & 0.864 & 0.605 & 34.98 & 60.9 & 27,4 & 19.1 & -- \\
\hline 0.711 & 0.897 & 0.864 & 0.605 & 48.42 & 84.3 & 34.7 & 26.0 & 33.5 \\
\hline 0.711 & 0.897 & 0.864 & 0.605 & 75.70 & 131.8 & 49.3 & 39.4 & - \\
\hline
\end{tabular}

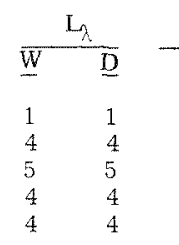

\begin{tabular}{ll}
$\mathrm{N}_{\lambda}$ & \multicolumn{1}{c}{} \\
\cline { 1 - 1 } & \\
4 & $1.25 \$$ \\
4 & 1.25 \\
4 & 1.25 \\
4 & 1.25 \\
3 & 1.25
\end{tabular}
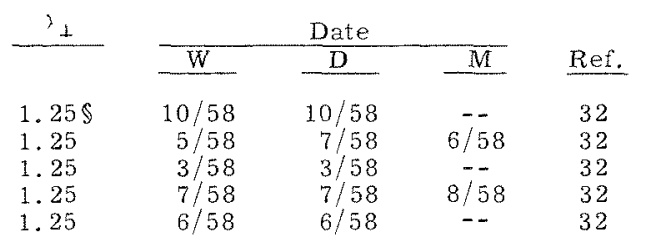

* Dry case included polyethylene spacers with an equivalent $\mathrm{V}-\mathrm{H}_{2} \mathrm{O} / \mathrm{U}$ of 0.011 .

$\$$ Measurements of $\lambda$ were made for several of the lattices but not all. Thus the

values were averaged to give 1.25 inches instead of being quoted as individual values. Clustered sources were used.

\section{TABLE 2}

CASES 2 through 5. 0.925-INCH CLUSTERS, 5-, 6-, OR 7-RODS, N.U.

\begin{tabular}{|c|c|c|c|c|c|c|c|c|c|c|c|c|c|c|c|c|c|}
\hline \multirow{2}{*}{$\begin{array}{l}\text { L.S. } \\
\text { (1n.) } \\
\end{array}$} & \multirow[b]{2}{*}{ Rods } & \multicolumn{2}{|c|}{$B_{11}$} & \multirow[b]{2}{*}{$\mathrm{V}-\mathrm{Al} / \mathrm{U}$} & \multirow[b]{2}{*}{$A-A 1 / U$} & \multirow{2}{*}{$\mathrm{V}-\mathrm{H}_{2} \mathrm{O} / \mathrm{U}$} & \multicolumn{3}{|c|}{ a. 8-Foot Pile } & \multicolumn{2}{|c|}{$\mathrm{CR}$} & \multicolumn{2}{|c|}{$B^{2}$} & \multicolumn{2}{|c|}{ Date } & \multirow[b]{2}{*}{ Ref. } & \multirow[b]{2}{*}{ N.B. } \\
\hline & & W & D & & & & $\mathrm{A}-\mathrm{H}_{2} \mathrm{O} / \mathrm{U}$ & $\mathrm{V}-\mathrm{C} / \mathrm{U}$ & $\mathrm{A}-\mathrm{C} / \mathrm{U}$ & W & D & $\mathrm{W}$ & D & $\mathrm{W}$ & $D$ & & \\
\hline \multicolumn{18}{|c|}{ Separated } \\
\hline 7 & 7 & 35.86 & 28.78 & 0.379 & 0.477 & 0.664 & 0.463 & 8.44 & 14.6 & 15.6 & 6.9 & -290 & -720 & -- & -- & 21,30 & -- \\
\hline $10-3 / 8$ & 7 & 40.51 & 41.21 & 0.379 & 0.477 & 0.664 & 0.463 & 21.23 & 36.9 & 28.9 & 18.0 & 2 & 23 & -- & -- & 21,30 & - \\
\hline 14 & 7 & 43.84 & 49.98 & 0.379 & 0.477 & 0.664 & 0.463 & 41.42 & 71.4 & 44.3 & 31.1 & -33 & 87 & -- & -- & 21,30 & -- \\
\hline $10-3 / 8$ & 5 & 40.05 & 42.66 & 0.427 & 0.537 & 1.004 & 0.701 & 29.95 & 52.0 & 31.5 & 22.2 & -12 & 62 & $5 / 56$ & $3 / 56$ & 21 & 40,26 \\
\hline & 5 & 53.11 & 70.25 & 0.427 & 0.537 & 1.004 & 0.701 & 57.94 & 100.0 & 57.9 & 39.6 & -69 & 83 & $1 / 56$ & $1 / 56$ & 21 & 41,26 \\
\hline \multicolumn{18}{|c|}{ Unseparated } \\
\hline & & 33.43 & 28.93 & 37 & 0.477 & 0.36 & 0.253 & 8.44 & 14.6 & 12.5 & & -407 & -708 & $\ldots$ & -- & 30 & -- \\
\hline $10-3 / 8$ & 7 & 41.30 & 40.89 & 0.367 & 0.477 & 0.362 & 0.253 & 21.40 & 37.2 & 25.8 & 18.7 & 25 & 13 & -- & $6 / 56$ & 30 & 40,26 \\
\hline 14 & 7 & 61.21 & 71.44 & 0.367 & 0.477 & 0.362 & 040 & 41.39 & 71.3 & 47.9 & 38.5 & 19 & 90 & $10 / 55$ & $12 / 55$ & 21,30 & 41,26 \\
\hline 14 & 6 & 59.99 & 71.03 & 0.394 & 0.496 & 0.631 & 0.440 & 48.30 & 83.4 & 50.3 & 40.6 & 8 & 87 & $10 / 55$ & $9 / 55$ & & 41,26 \\
\hline
\end{tabular}


TABLE 3

CASE 1. $1.36 \times 0.50$-INCH HOLLOW, N.U. a. 8-Foot Pile (E Only)

\begin{tabular}{|c|c|c|c|}
\hline \multirow{2}{*}{$\begin{array}{l}\text { L. S. } \\
\text { (in.) }\end{array}$} & \multicolumn{2}{|c|}{$B_{11}$} & \multirow[b]{2}{*}{$\mathrm{V}-\mathrm{Al} / \mathrm{U}$} \\
\hline & $\mathrm{W}$ & D & \\
\hline $5-3 / 16$ & 48.97 & 45.65 & 0.554 \\
\hline $10-3 / 8$ & $\begin{array}{l}57.69 \\
57.59\end{array}$ & 62.60 & $\begin{array}{r}.554 \\
0.554\end{array}$ \\
\hline
\end{tabular}

\section{$\mathrm{A}-\mathrm{AL} / \mathrm{U}$} 0.703 0.703
0.703

\section{$\mathrm{V}-\mathrm{H}_{2} \mathrm{O} / \mathrm{U}$ 0.314} 0.314

\section{$\underline{\mathrm{A}-\mathrm{H}} 2 \mathrm{O} / \mathrm{U}$ 0.221} 0.221
$\frac{\mathrm{V}-\mathrm{C} / \mathrm{U}}{21.85} \quad \frac{\mathrm{A}-\mathrm{C} / \mathrm{U}}{38.3}$

$\begin{array}{ll}21.85 & 38.3 \\ 41.08 & 71.5 \\ 92.2 & 11.5\end{array}$

\section{$\frac{\mathrm{CR}}{\mathrm{W}} \frac{\mathrm{D}}{16.5}$} $\begin{array}{ll}16.5 & 12.4 \\ 28.7 & 23.6\end{array}$ $\begin{array}{ll}28.7 & 23.6 \\ 58.7 & 51.8\end{array}$

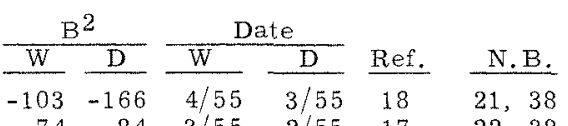
$\begin{array}{llllll}74 & 84 & 3 / 55 & 2 / 55 & 17 & 22,38\end{array}$

TABLE 3

CASE 1. $1.36 \times 0.50-$ INCH HOLLOW, N.U. b. 4-Foot Prle (E Only)

0.221

$59.47 \quad 104.25$

38.2

$84 \quad-\quad 6 / 54 \quad \ldots \quad \ldots$

16,38

TABLE 3

CASE 2. $1.37 \times 0.48$-INCH HOLLOW, N.U.

$$
\text { a. 4-Foot Pile }
$$

$350 \quad 56.6 \quad 98.2$

$\begin{array}{ll}0.350 & 56 . \\ 0.350 & 47.3\end{array}$

98.2
82.2

$\begin{array}{lllllll}40.4 & 33.1 & 74 & 127 & 4 / 57 & 4 / 57 & * \\ 33.5 & 26.8 & 77 & 125 & 4 / 57 & 4 / 57 & *\end{array}$

TABLE 3

CASE 3. $1.37 \times 0.48$-INCH HOLLOW, 1.44 PER CENT ENRICHMENT

$$
\text { a. 4-Foot Pile }
$$

$\begin{array}{cccc}5-3 / 16 & 34.49 & 30.06 & 0.473 \\ 6-3 / 16 & 44.44 & 39.18 & 0.473 \\ 7-3 / 16 & 42.99 & 41.18 & 0.473 \\ 7-11 / 16 & 43.46 & 42.60 & 0.473 \\ 8-3 / 8 & 38.53 & 38.60 & 0.473 \\ 9-3 / 8 & 47.19 & 49.31 & 0.473 \\ 10-3 / 8 & 34.33 & 35.58 & 0.473 \\ 8-3 / 8 & 51.81 & 52.39 & 0.654\end{array}$

$\begin{array}{llllr}0.600 & 0.501 & 0.350 & 20.4 & 35.5 \\ 0.600 & 0.501 & 0.350 & 29.9 & 52.0 \\ 0.600 & 0.501 & 0.350 & 41.1 & 71.4 \\ 0.600 & 0.501 & 0.350 & 47.3 & 82.2 \\ 0.600 & 0.501 & 0.350 & 56.6 & 98.2 \\ 0.600 & 0.501 & 0.350 & 71.2 & 123.9 \\ 0.600 & 0.501 & 0.350 & 87.7 & 152.5 \\ 0.823 & 0.447 & 0.312 & 56.5 & 98.0\end{array}$

$\begin{array}{rr}12.7 & 8.4 \\ 17.4 & 12.6 \\ 22.9 & 17.6 \\ 25.6 & 20.4 \\ 30.5 & 25.0 \\ 35.5 & 29.9 \\ 42.6 & 35.8 \\ 28.6 & 24.5\end{array}$

$\begin{array}{rrrrr}739 & 473 & 11 / 56 & 11 / 56 & 6 \\ 711 & 566 & 4 / 57 & 4 / 57 & 6 \\ 663 & 614 & 1 / 57 & 1 / 57 & 6 \\ 630 & 609 & 1 / 57 & 1 / 57 & 6 \\ 585 & 588 & 11 / 56 & 11 / 56 & 6 \\ 499 & 537 & 1 / 57 & 1 / 57 & 6 \\ 420 & 478 & 11 / 56 & 11 / 56 & 6 \\ 572 & 580 & 4 / 57 & 4 / 57 & *\end{array}$

42,31 39 33
28 24
25

$31, \quad 32$

* HW-49752 B

Extrapolation distance used was 1 inch for Case $1-\mathrm{a}, 1.6$ inches for Case $1-\mathrm{b}$, and 1.15 inches for Cases 2 and 3.

Clustered sources were used for Cases $1-b, 2$, and 3 . 
TABLE 3

CASE 4. $1.66 \times 0.81-\mathrm{INCH}$ HOLLOW, N.U.

a. 8-Foot Pile (I Only)

\begin{tabular}{|c|c|c|c|c|c|c|c|c|c|c|c|c|c|c|c|c|}
\hline \multirow{2}{*}{$\begin{array}{l}\text { L.S. } \\
\text { (in.) }\end{array}$} & \multicolumn{2}{|c|}{$B_{11}$} & \multirow[b]{2}{*}{$\mathrm{V}-\mathrm{Al} / \mathrm{U}$} & \multirow[b]{2}{*}{$\mathrm{A}-\mathrm{A} 1 / \mathrm{U}$} & \multirow[b]{2}{*}{$\mathrm{V}-\mathrm{H}_{2} \mathrm{O} / \mathrm{U}$} & \multirow[b]{2}{*}{$\mathrm{A}-\mathrm{H}_{2} \mathrm{O} / \mathrm{U}$} & \multirow[b]{2}{*}{$\mathrm{V}-\mathrm{C} / \mathrm{U}$} & \multirow[b]{2}{*}{$A-C / U$} & \multicolumn{2}{|c|}{$\mathrm{CR}$} & \multicolumn{2}{|c|}{$\mathrm{B}^{2}$} & \multicolumn{2}{|c|}{$\mathrm{Da}$} & \multirow[b]{2}{*}{ Ref. } & \multirow[b]{2}{*}{ N.B. } \\
\hline & $W$ & D & & & & & & & $\bar{W}$ & D & W & $\mathrm{D}$ & W & D & & \\
\hline $\begin{array}{l}6-3 / 16 \\
7-1 / 2\end{array}$ & $\begin{array}{l}52.11 \\
71.48\end{array}$ & $\begin{array}{l}5 \\
7\end{array}$ & 0.175 & 222 & $\begin{array}{l}0.224 \\
0.224\end{array}$ & $\begin{array}{l}0.158 \\
0.158\end{array}$ & $\begin{array}{l}21.78 \\
32.68\end{array}$ & $\begin{array}{l}16 \\
96\end{array}$ & $\begin{array}{l}5 \\
2\end{array}$ & $\begin{array}{l}14.3 \\
21.9\end{array}$ & $\begin{array}{r}-53 \\
93\end{array}$ & $\begin{array}{r}-66 \\
98\end{array}$ & $\begin{array}{r}9 / 53 \\
10 / 53\end{array}$ & $\begin{array}{l}9 / 53 \\
9 / 53\end{array}$ & $\begin{array}{l}4 \\
4\end{array}$ & $10, \quad 27$ \\
\hline $\begin{array}{l}1 / 2 \\
3 / 8\end{array}$ & 66.57 & 69. & & $\begin{array}{l}2 \\
2\end{array}$ & 0.224 & 0 . & $\begin{array}{l}36.08 \\
63.86\end{array}$ & $\begin{array}{r}50 \\
111\end{array}$ & 43 & $\begin{array}{l}21 \\
42\end{array}$ & $\begin{array}{l}83 \\
88\end{array}$ & $\begin{array}{r}98 \\
109\end{array}$ & $\begin{array}{r}10 / 53 \\
9 / 54\end{array}$ & 9 & - & $\begin{array}{ll}11, & 27 \\
20, & 27\end{array}$ \\
\hline $2-$ & 59.74 & 61.51 & 0.175 & 0.222 & 0.224 & 0.158 & 91.46 & 160.24 & 58.6 & 57.1 & 35 & 51 & $10 / 53$ & $5 / 54$ & 4 & 10,27 \\
\hline & 54.81 & 56.07 & 0.175 & 0.222 & 0.224 & 0.158 & 135.06 & 235.41 & 81.8 & 80.7 & -41 & -30 & $10 / 53$ & $11 / 53$ & 4 & 11,27 \\
\hline
\end{tabular}

TABLE 3

CASE $5.1 .66 \times 0.94-$ INCH HOLLOW, 1.007 PER CENT ENRICHMENT *

a. 4-Foot Pile (I Only)

\begin{tabular}{|c|c|c|c|c|c|c|c|c|c|c|c|c|c|c|c|c|}
\hline $6-3 / 16$ & 33.12 & 32.20 & 0.196 & 0.247 & 0.251 & 0.175 & 24.43 & 42.48 & -- & -- & 306 & 253 & $3 / 56$ & $3 / 56$ & 6 & 39 , \\
\hline $7-1 / 2$ & 35.60 & 34.86 & 0.196 & 0.247 & 0.251 & 0.175 & 36.65 & 63.29 & -- & - & 409 & 375 & $2 / 56$ & $2 / 56$ & 6 & 43 \\
\hline $8-3 / 8$ & $\ldots$ & 33.75 & 0.196 & 0.247 & 0.251 & $\ldots$ & 46.11 & 80.19 & -- & 24.8 & - & 380 & -- & $7 / 56$ & 6 & 24 , \\
\hline $10-3 / 8$ & 32.56 & 32.72 & 0.196 & 0.247 & 0.251 & 0.175 & 71.62 & 124.45 & 37.2 & 36.0 & 324 & 333 & $7 / 56$ & $6 / 56$ & 6 & 29, \\
\hline $12-3 / 8$ & 31.76 & 31.70 & 0.196 & 0.247 & 0.251 & 0.175 & 102.59 & 178.40 & -- & -- & 226 & 222 & $3 / 56$ & $3 / 56$ & 6 & 44, \\
\hline $10-3 / 8 \S$ & -- & $\ldots$ & 0.163 & 0.21 & 0.397 & 0.279 & 71.62 & 124.45 & -- & -- & 331 & 332 & $7 / 56$ & $6 / 56$ & - & \\
\hline $7-1 / 2^{\text {类党 }}$ & 35.60 & 34.93 & 0.102 & 0.128 & -.. & $\ldots$ & 36.65 & 63.29 & 19.69 & -- & 409 & 378 & $2 / 56$ & $2 / 56$ & - & \\
\hline
\end{tabular}

* Extrapolation distance used was 1.15 inches. Clustered sources were used.

* No inner can used.

wh th shightly-changed volumes of water and aluminum. No information avallable

on geometry of cans and water.

TABLE 3

CASE 6. 1.66 × 1.12-INCH HOLLOW, N.U. a. 8-Foot Pile (I Only)

\begin{tabular}{|c|c|c|c|c|c|c|c|c|c|c|c|c|c|c|c|c|}
\hline $3 / 16$ & 56.21 & 56.57 & 0.283 & 0.362 & 0.652 & 0.463 & 30.43 & 53.77 & 20.6 & 16.7 & -1 & 3 & $5 / 54$ & $1 / 54$ & 4 & 10 \\
\hline$-1 / 2$ & 68.19 & 73.51 & 0.283 & 0.362 & 0.652 & 0.463 & 45.66 & 80.68 & 28.7 & 26.1 & 73 & 103 & $4 / 54$ & $3 / 54$ & 4 & \\
\hline $10-3 / 8$ & 59.36 & 64.54 & 0.283 & 0.362 & 0.652 & 0.463 & 89.24 & 157.69 & 51.2 & 48.6 & 29 & 73 & $10 / 54$ & $9 / 54$ & 4 & 20 , \\
\hline $12-3 / 8$ & 54.15 & 57.64 & 0.283 & 0.362 & 0.652 & 0.463 & 127.90 & 226.00 & 71.0 & 65.8 & -26 & 14 & $5 / 54$ & $1 / 54$ & 4 & 10, \\
\hline 15 & 51.01 & 53.47 & 0.283 & 0.362 & 0.652 & 0.463 & 188.74 & 333.50 & 98.2 & 96.9 & -96 & -61 & $3 / 54$ & $3 / 54$ & 4 & 11 , \\
\hline
\end{tabular}


TABLE 3

CASE 7. $2.5 \times 1.6$-INCH HOLLOW, N.U.

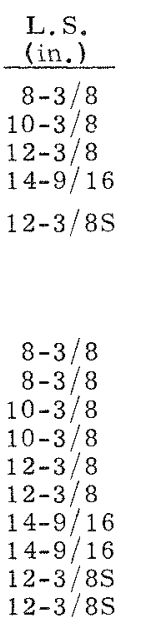

\begin{tabular}{cc}
\multicolumn{2}{c}{$\mathrm{B}_{11}$} \\
\hline $\begin{array}{c}\mathrm{W} \\
31.45\end{array}$ & $\frac{\mathrm{D}}{31.58}$ \\
33.03 & 35.92 \\
33.33 & 36.63 \\
31.44 & 35.03 \\
33.37 & 36.37
\end{tabular}

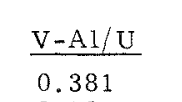

0.381
0.381

0.381

0.381

0.381

$\frac{\mathrm{A}-\mathrm{A} 1 / \mathrm{U}}{0.494}$

0.494

0.494

0.494

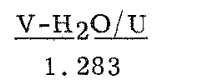

$\frac{\mathrm{A}-\mathrm{H}_{2} \mathrm{O} / \mathrm{U}}{0.907}$

$\frac{\mathrm{V}-\mathrm{C} / \mathrm{U}}{21.47}$

1.283

0.907

$34.47 \quad 37.74$

$\begin{array}{ll}34.41 & 60.49 \\ 50.11 & 88.09\end{array}$

70.44

88.09
123.83

0.907

50.11

88.09

\begin{tabular}{|c|c|c|c|}
\hline \multicolumn{2}{|c|}{$\mathrm{CR}$} & \multicolumn{2}{|c|}{$\mathrm{B}^{2}$} \\
\hline$\overline{\mathrm{W}}$ & $\bar{D}$ & W & $\mathrm{D}$ \\
\hline 30.3 & 17.1 & -68 & -65 \\
\hline 37.4 & 22.9 & -34 & 100 \\
\hline 57.9 & 36.8 & -43 & 111 \\
\hline- & 46.0 & -129 & 70 \\
\hline 56.9 & 35.8 & -32 & 114 \\
\hline
\end{tabular}

\begin{tabular}{c} 
Coolant \\
\hline D \\
W \\
D \\
W \\
D \\
W \\
D \\
W \\
D \\
W
\end{tabular}

\begin{tabular}{c}
$\mathbf{L}_{\lambda}$ \\
\hline 3 \\
3 \\
$1+2$ \\
$1+2$ \\
3 \\
3 \\
$3 *$ \\
$3 *$ \\
3 \\
3
\end{tabular}

\begin{tabular}{rll}
$\frac{\mathrm{N}_{\lambda}}{14}$ & & \multicolumn{1}{c}{$\lambda_{\perp}$} \\
14 & & 1.5 \\
7 & & 1.5 \\
7 & & 1.8 \\
10 & 2.4 \\
12 & 0.7 \\
8 & 1.9 \\
8 & 2.1 \\
10 & & 1.7 \\
10 & & 1.6
\end{tabular}

$\begin{array}{lll}\frac{\lambda \|}{1.03} & & \frac{\sigma(\lambda)}{0.084} \\ 1.03 & & 0.99 \\ 1.03 & & 0.74 \\ 1.03 & & 0.35 \\ 1.03 & & 0.34 \\ 1.03 & & 0.13 \\ 1.03 & & 0.18 \\ 1.03 & & 0.22 \\ 1.2 & & 0.18 \\ 1.03 & & 0.15\end{array}$

\begin{tabular}{c}
$\sigma\left(\mathrm{B}^{2}\right)$ \\
\hline 6 \\
4 \\
7 \\
7 \\
3 \\
4 \\
5 \\
6 \\
3 \\
3
\end{tabular}

* Taken 1 inch above position 3.

Clustered sources were used except for cases labeled " $\mathrm{S}$ " which were rerun with split sources. 
CASES 1 AND 2. $1.356 \times 0.414-I N C H$ HOLLOW, N.U. a. Comparison with 1.336-Inch Solid, 4-Foot Pile

\begin{tabular}{|c|c|c|c|c|c|c|c|c|}
\hline Case & $\begin{array}{l}\text { L.S. } \\
\text { (in.) }\end{array}$ & $\mathrm{V}-\mathrm{Al} / \mathrm{U}$ & $\underline{A-A} 1 / U$ & $\mathrm{~V}-\mathrm{H}_{2} \mathrm{O} / \mathrm{U}$ & $\mathrm{A}-\mathrm{H}_{2} \mathrm{O} / \mathrm{U}$ & $\mathrm{V}-\mathrm{C} / \mathrm{U}$ & $\underline{A-C / U}$ & $\frac{\mathrm{B}^{2}}{\underline{\mathrm{W}}}$ \\
\hline 1 (Hollow) & $8-3 / 8$ & 0.605 & 0.762 & 0.319 & 0.223 & 55.56 & 96.62 & 42 \\
\hline $2($ Solıd $)$ & $8-3 / 8$ & 0.522 & 0.657 & 0.273 & 0.200 & 51.88 & 90.22 & 54 \\
\hline & & & & \multicolumn{5}{|c|}{ TABLE 3(a) } \\
\hline & & & & \multicolumn{5}{|c|}{ CASES 3 AND $4.1 .37 \times 0.48$-INCH HOLLOW, N.U. } \\
\hline & & & & \multicolumn{5}{|c|}{ a. Comparison with 1.336-Inch Solid, 4-Foot Pile } \\
\hline 3 (Hollow) & $8-3 / 8$ & 0.622 & 0.783 & 0.398 & 0.287 & 56.15 & 97.64 & \\
\hline 4. (Solid) & $8-3 / 8$ & $0 \quad 523$ & $0 \quad 658$ & $0 \quad 334$ & $0 \quad 233$ & 5178 & 90.04 & \\
\hline
\end{tabular}

\begin{tabular}{cr}
$\frac{\text { Differences }}{\text { Dry (Solıd) - Wet (Solıd) }}$ & \multicolumn{1}{c}{$\frac{\Delta \mathrm{B}^{2}}{33.3 \pm 2.2}$} \\
Dry (I \& E) - Wet (I \& E) & $40.0 \pm 2.2$ \\
Dry (Solıd) - Dry (I \& E) & $5.0 \pm 2.5$ \\
Wet (Solnd) - Wet (I \& E) & $11.7 \pm 19$
\end{tabular}

References HW-49752 B, HW-57772, and undocumented letter, "Reactivity Changes with

I \& E Fuel Elements", E. Z. Block to J. H. Brown, 5-27-57.

Extrapolation distance used was 1.66 inches for this series. No attempt was made to

determine accurate '"s since the study was intended to measure differences, not the

absolute values of the buckling.

Clustered sources were used for all four cases. 
TABLE 4

CASE $1.2 .5 \times 1.6$-INCH TUBE WITH 0.5 -INCH ROD, N.U. *** a. 4-Foot Pile

\begin{tabular}{|c|c|c|c|c|c|c|c|c|c|c|c|c|c|}
\hline \multirow{2}{*}{$\begin{array}{l}\text { L.S.S. } \\
\text { (mn.) }\end{array}$} & \multicolumn{2}{|c|}{$\mathrm{B}_{11}$} & \multirow[b]{2}{*}{$\mathrm{V}-\mathrm{A} 1 / \mathrm{U}$} & \multirow[b]{2}{*}{$\mathrm{A}-\mathrm{A} \perp / \mathrm{U}$} & \multirow[b]{2}{*}{$\mathrm{V}-\mathrm{H}_{2} 2 \mathrm{O} / \mathrm{U}$} & \multirow[b]{2}{*}{$\mathrm{A}-\mathrm{H}_{2} 2 \mathrm{O} / \mathrm{U}$} & \multirow{2}{*}{\multicolumn{2}{|c|}{$\mathrm{V}-\mathrm{C} / \mathrm{U}$}} & \multirow[b]{2}{*}{$\underline{A-C / U}$} & \multicolumn{2}{|c|}{$\mathrm{CR}$} & \multicolumn{2}{|c|}{$\mathrm{B}^{2}$} \\
\hline & W & $\mathrm{D}$ & & & & & & & & W & D & $\overline{\mathrm{W}}$ & D \\
\hline $14-9 / 16$ & 31.71 & 34.87 & 0.382 & 0.484 & 1.11 & 0.781 & & & 114.94 & 69.3 & 48.0 & -111 & 63 \\
\hline & & & $\underline{\mathrm{N}_{\mathrm{B}}}$ & $\underline{\mathrm{L}_{\lambda}}$ & $\underline{N_{\lambda}}$ & $\lambda_{L}$ & $\lambda_{11}$ & & & $\underline{\sigma\left(B^{2}\right)}$ & Date & & Ref. \\
\hline $\begin{array}{l}14-9 / 16 \\
14-9 / 16\end{array}$ & & & $\begin{array}{l}4 \\
4\end{array}$ & $\begin{array}{l}3^{*} \\
3^{*}\end{array}$ & $\begin{array}{l}8 \\
8\end{array}$ & $\begin{array}{l}1.9 \\
2.0\end{array}$ & $\begin{array}{l}1.03 \\
1.03\end{array}$ & & & $\begin{array}{l}4 \\
4\end{array}$ & $\begin{array}{l}8 / 59 \\
9 / 59\end{array}$ & & $\begin{array}{l}37 \\
37\end{array}$ \\
\hline
\end{tabular}

* Taken 1 inch above position 3 .

$*$ Clustered sources were used.

TABLE 4

CASE 2. $2.5 \times 1.6$-INCH TUBE WITH 1.175 -INCH ROD, N.U.*** a. 4-Foot Pile

\begin{tabular}{|c|c|c|}
\hline \multirow{2}{*}{$\begin{array}{l}\text { L.S. } \\
\text { (in.) }\end{array}$} & \multicolumn{2}{|c|}{$B_{11}$} \\
\hline & W & $\mathrm{D}$ \\
\hline $8-3 / 8$ & 31.66 & 30.75 \\
\hline $10-3 / 8$ & 33.96 & 35.59 \\
\hline $12-3 / 8$ & 33.76 & 36.95 \\
\hline $14-9 / 16$ & 32.27 & 35.07 \\
\hline \multirow[t]{2}{*}{$12-3 / 85$} & 33.10 & -- \\
\hline & \multicolumn{2}{|c|}{ Coolant } \\
\hline $8-3 / 8$ & \multicolumn{2}{|c|}{ D } \\
\hline $8-3 / 8$ & \multicolumn{2}{|c|}{ W } \\
\hline $10-3 / 8$ & \multicolumn{2}{|c|}{$\mathrm{D}$} \\
\hline $10-3 / 8$ & \multicolumn{2}{|c|}{ W } \\
\hline $12-3 / 8$ & \multirow{2}{*}{\multicolumn{2}{|c|}{$\underset{W}{\mathrm{D}}$}} \\
\hline $12-3 / 8$ & & \\
\hline $14-9 / 16$ & \multicolumn{2}{|c|}{$\mathrm{D}$} \\
\hline $14-9 / 16$ & \multirow{2}{*}{\multicolumn{2}{|c|}{ W }} \\
\hline $12-3 / 8 S$ & $\mathrm{~W}$ & \\
\hline
\end{tabular}

\begin{tabular}{|c|c|}
\hline $\mathrm{V}-\mathrm{Al} / \mathrm{U}$ & $\mathrm{A}-\mathrm{Al} / \mathrm{U}$ \\
\hline 0.311 & 0.393 \\
\hline 0.311 & 0.393 \\
\hline 0.311 & 0.393 \\
\hline 0.311 & 0.393 \\
\hline 0.311 & 0.393 \\
\hline $\mathrm{N}_{\mathrm{B}}$ & $\underline{L_{\lambda}}$ \\
\hline 6 & 3 \\
\hline 5 & 3 \\
\hline 6 & $1+2$ \\
\hline 5 & $1+2$ \\
\hline 4 & 3 \\
\hline 6 & 3 \\
\hline 4 & 5 \\
\hline 4 & $2^{*}$ \\
\hline 5 & 3 \\
\hline
\end{tabular}

$\begin{array}{rl}\frac{\mathrm{V}-\mathrm{H}_{2}}{0} \mathrm{O} / \mathrm{U} & \\ 0.626 & \\ 0.626 & \\ 0.626 & \\ 0.626 & \\ 0.626 & \\ & \\ \mathrm{~N}_{\lambda} & \\ 14 & \\ 14 & \\ 7 & 1.5 \\ 7 & 1.5 \\ 10 & 1.7 \\ 10 & 2.4 \\ 8 & 2.4 \\ 8 & 1.5 \\ 10 & 1.1\end{array}$

$\begin{array}{lll}\frac{\mathrm{A}-\mathrm{H}_{2}}{2} \mathrm{O} / \mathrm{U} & & \frac{\mathrm{V}-\mathrm{C} / \mathrm{U}}{\mathrm{A}-\mathrm{C} / \mathrm{U}} \\ 0.439 & & \frac{15.62}{27.15} \\ 0.439 & 25.03 & 43.52 \\ 0.439 & 36.46 & 63.40 \\ 0.439 & 51.25 & 89.12 \\ 0.439 & 36.46 & 63.40\end{array}$

\begin{tabular}{|c|c|c|c|}
\hline \multicolumn{2}{|c|}{$\mathrm{CR}$} & \multicolumn{2}{|c|}{$B^{2}$} \\
\hline $\mathrm{W}$ & $\mathrm{D}$ & $\overline{\mathrm{W}}$ & $\mathrm{D}$ \\
\hline 25.2 & -1 & -51 & -111 \\
\hline 36.3 & 21.0 & 7 & 87 \\
\hline 49.3 & 33.2 & -21 & 126 \\
\hline 61.0 & -- & -68 & 65 \\
\hline 49.1 & -- & -31 & - \\
\hline
\end{tabular}

* Taken 1 inch above position 2

\begin{tabular}{|c|c|c|}
\hline$\lambda_{1}$ & $\lambda_{11}$ & $\sigma(\lambda)$ \\
\hline 1.5 & 1.03 & 0.11 \\
\hline 1.5 & 1.03 & 0.085 \\
\hline 1.5 & 1.03 & 0.63 \\
\hline 1.7 & 1.03 & 0.27 \\
\hline 2.4 & 1.03 & 0.24 \\
\hline 2.4 & 1.03 & 0.23 \\
\hline 2.2 & 1.03 & 0.70 \\
\hline 1.5 & 1.03 & 0.22 \\
\hline 1. & 0.87 & 0.28 \\
\hline
\end{tabular}

\begin{tabular}{|c|c|}
\hline$\sigma(\mathrm{B} 2)$ & Date \\
\hline 7 & $12 / 59$ \\
\hline 6 & $12 / 59$ \\
\hline 7 & $2 / 60$ \\
\hline 7 & $3 / 60$ \\
\hline 3 & $2 / 61$ \\
\hline 2 & $2 / 61$ \\
\hline 6 & $7 / 59$ \\
\hline 5 & $9 / 59$ \\
\hline 5 & $1 / 61$ \\
\hline
\end{tabular}

\begin{tabular}{c} 
Ref. \\
\hline 37 \\
37 \\
37 \\
37 \\
41 \\
41 \\
37 \\
37 \\
41
\end{tabular}

** Clustered sources were used except for case labeled "S". 
TABLE 5

CASE $1.2 .5 \times 2.0$-INCH TUBE WITH $1.66 \times 1.12$-INCH TUBE, N.U.**

\begin{tabular}{l}
$\frac{\text { L.S. }}{\text { (in.) }}$ \\
\hline $7-3 / 16$ \\
$8-3 / 8$ \\
$10-3 / 8$ \\
$12-3 / 8$ \\
$14-9 / 16$
\end{tabular}

\begin{tabular}{|c|c|}
\hline \multicolumn{2}{|c|}{$\mathrm{B}_{11}$} \\
\hline $\bar{W}$ & D \\
\hline 30.59 & 28.88 \\
\hline 31.44 & 31.74 \\
\hline 31.73 & 34.02 \\
\hline 32.70 & 36.24 \\
\hline 31.52 & 34.67 \\
\hline
\end{tabular}

\begin{tabular}{l}
$\mathrm{V}-\mathrm{Al} / \mathrm{U}$ \\
\hline 0.502 \\
0.502 \\
0.502 \\
0.502 \\
0.502
\end{tabular}

\begin{tabular}{l}
$\mathrm{A}-\mathrm{Al} / \mathrm{U}$ \\
\hline 0.634 \\
0.634 \\
0.634 \\
0.634 \\
0.634
\end{tabular}

\begin{tabular}{l}
$\frac{a .4-\mathrm{F}}{\mathrm{V}-\mathrm{H}_{2} \mathrm{O} / \mathrm{U}}$ \\
\hline 1.094 \\
1.094 \\
1.094 \\
1.094 \\
1.094
\end{tabular}

Foot Pile

$\begin{array}{lc} & \text { Coolant } \\ 7-3 / 16 & \mathrm{~W} \\ 7-3 / 16 & \mathrm{D} \\ 8-3 / 8 & \mathrm{~W} \\ 8-3 / 8 & \mathrm{D} \\ 10-3 / 8 & \mathrm{~W} \\ 10-3 / 8 & \mathrm{D} \\ 12-3 / 8 & \mathrm{~W} \\ 12-3 / 8 & \mathrm{D} \\ 14-9 / 16 & \mathrm{~W} \\ 14-9 / 16 & \mathrm{D}\end{array}$

$\begin{array}{cc}\frac{\mathrm{N}_{\mathrm{B}}}{5} & \frac{\mathrm{L}_{\lambda}}{4} \\ 4 & 1 \\ 6 & 3 \\ 6 & 3 \\ 5 & 1+2 \\ 5 & 1+2 \\ 5 & 1+2 \\ 5 & 1+2 \\ 4 & 3 * \\ 4 & 3 *\end{array}$

\begin{tabular}{|c|c|}
\hline $\mathrm{N}_{\lambda}$ & ${ }^{\lambda_{1}}$ \\
\hline 8 & 1.4 \\
\hline 8 & 1.6 \\
\hline 7 & 1.3 \\
\hline 14 & 1.5 \\
\hline 11 & 1.4 \\
\hline 10 & 1.8 \\
\hline 11 & 1.5 \\
\hline 11 & 1.5 \\
\hline 4 & 2.2 \\
\hline 4 & 1.8 \\
\hline
\end{tabular}

$\frac{\lambda 11}{1.03}$
1.03
1.03
1.03
1.03
1.03
1.03
1.03
1.03
1.03

$\frac{o(\lambda)}{0.13}$
0.11
0.15
0.09
0.14
0.25
0.14
0.15
.-
--

* Taken 3 inches above position 3 .

** Clustered sources were used.

\begin{tabular}{|c|c|c|c|}
\hline \multicolumn{2}{|c|}{$\mathrm{CR}$} & \multicolumn{2}{|c|}{$B^{2}$} \\
\hline $\mathrm{W}$ & $\bar{D}$ & $\mathrm{~W}$ & $\overline{\mathrm{D}}$ \\
\hline 23.00 & 10.09 & -123 & -259 \\
\hline 28.27 & 14. & -64 & -50 \\
\hline 38.24 & 25. & -45 & 76 \\
\hline 56.16 & 36.86 & -72 & 104 \\
\hline 65.32 & $\ldots$ & -127 & 55 \\
\hline
\end{tabular}

TABLE 5

CASE 1. $2.5 \times 2.0$-INCH TUBE WITH $1.66 \times 1.12$-INCH TUBE, N.U.

$$
\text { b. } 8 \text {-Foot Pile }
$$

\begin{tabular}{c} 
L.S. \\
(in.) \\
\hline $8-3 / 8$ \\
$8-3 / 8$ \\
$14-9 / 16$ \\
$8-3 / 8$ \\
$8-3 / 8$ \\
$14-9 / 16$
\end{tabular}

\begin{tabular}{l}
$\begin{array}{c}\text { Pile Size } \\
\text { (feet) }\end{array}$ \\
\hline $6 \times 8$ \\
$8 \times 8$ \\
$10 \times 8$ \\
$6 \times 8$ \\
$8 \times 8$ \\
$10 \times 8$
\end{tabular}

Source
Position
Clustered
Clustered
Clustered
Split
Split
Split

$\lambda 1$
1.6
2.0
3.7
1.0
0.8
0.7

$\frac{\lambda 11}{1.9}$
2.1
1.5
1.1
1.2
0.7

\begin{tabular}{cc}
$\frac{B^{2}}{W}$ & Ref. \\
\hline-63 & 39 \\
-63 & 39 \\
-123 & 39 \\
-72 & 39 \\
-87 & 39 \\
-128 & 39
\end{tabular}




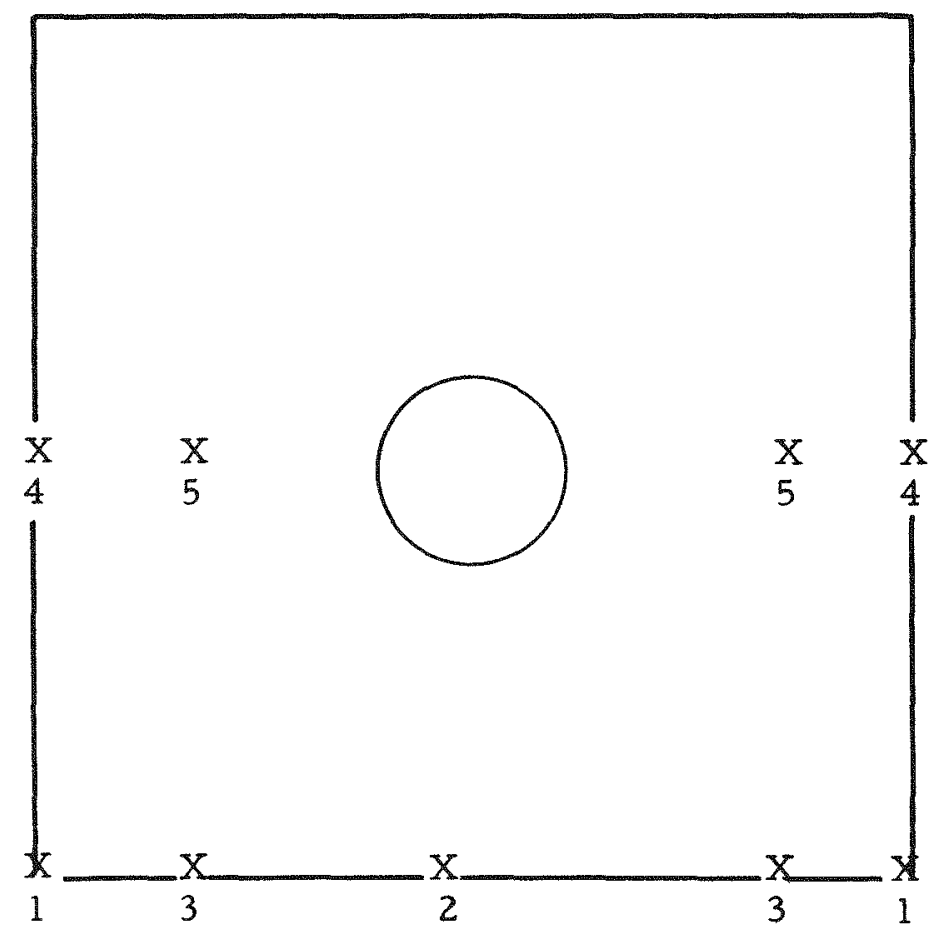

Position

1 - Cell corner, between columns of tubes.

2 - Cell edge, under columns of tubes.

3 - Cell edge, radius of equivalent cylindrical cell (approximate).

4 - Between tubes, tube row.

5 - Corresponding positions, tube row.

FIGURE II-1

Cell Position for Horizontal Traverses 
EXPONENTIAL PILE CONSTRUCTION DIAGRAMS 
TABLE 1

LATTICE SPACINGS USED FOR BUCKLING MEASUREMENTS

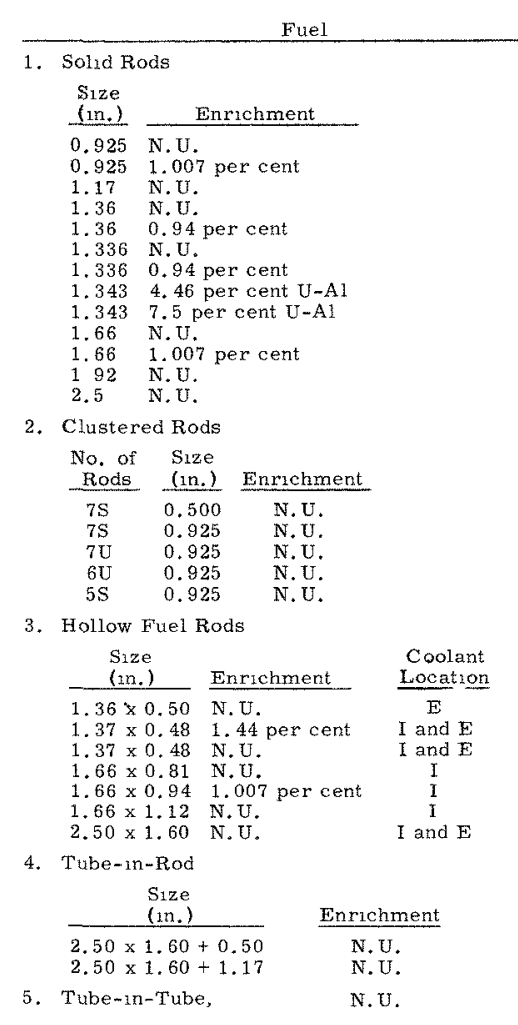

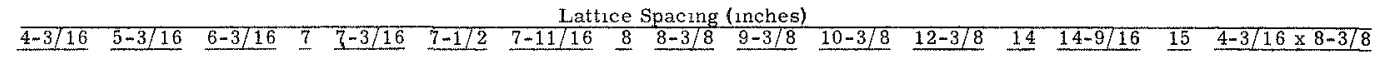

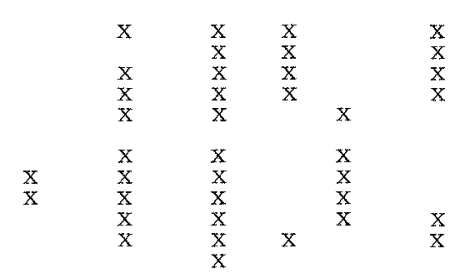

$\mathrm{x}$

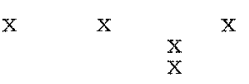

$\frac{x}{x}$

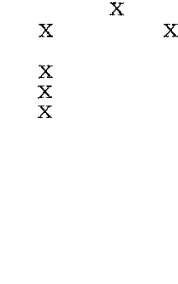

$\mathrm{X}$
$\mathrm{X}$
$\mathrm{X}$
$\mathrm{X}$
$\mathrm{X}$
$\mathrm{X}$
$\mathrm{X}$
$\mathrm{X}$
$\mathrm{X}$
$\mathrm{X}$
$\mathrm{X}$
$\mathrm{X}$
$\mathrm{X}$
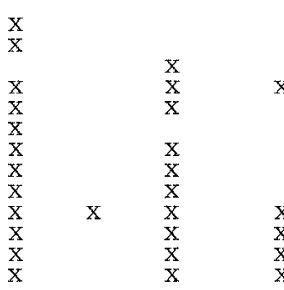

㤐 
Fuel Element:

$1.343 ", 4.46 \% \mathrm{U}-\mathrm{Al}$ Alloy

The $1.343^{\prime \prime}, 7.5 \%$ rod has the same construction except for two less rows at the top.

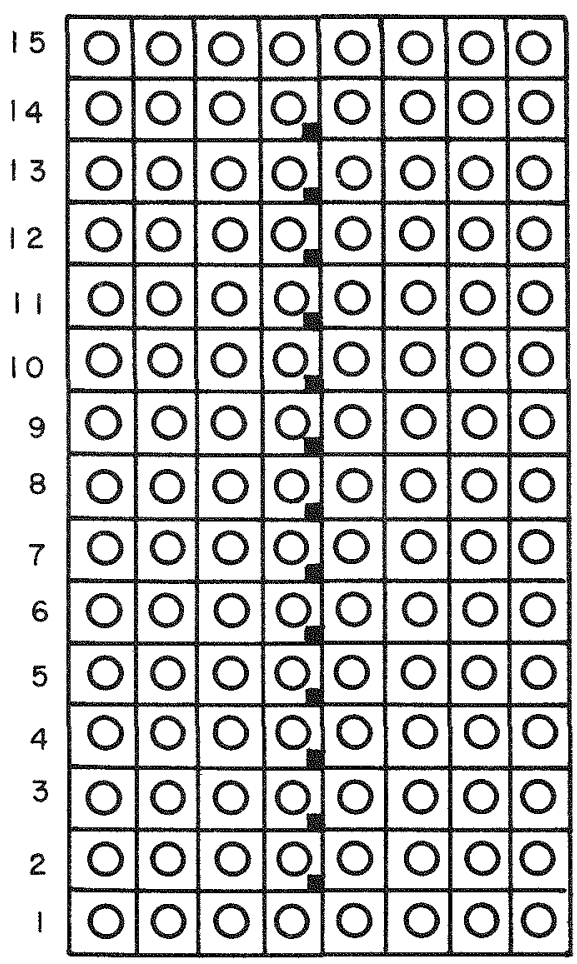

\begin{tabular}{|c|c|}
\hline Slot & $\mathrm{Z}(\mathrm{cm})$ \\
\hline 2 & 58.34 \\
\hline 3 & 68.98 \\
\hline 4 & 79.61 \\
\hline 5 & 90.25 \\
\hline 6 & 100.88 \\
\hline 7 & 111.52 \\
\hline 8 & 122.16 \\
\hline 9 & 132.79 \\
\hline 10 & 143.43 \\
\hline 11 & 154.06 \\
\hline 12 & 164.70 \\
\hline 13 & 175.34 \\
\hline 14 & 185.97 \\
\hline$x=$ & $.27 \mathrm{~cm}$ \\
\hline 7 & \\
\hline
\end{tabular}

$a=85.09 \mathrm{~cm}$

$\mathrm{b}=121.92 \mathrm{~cm}$

$\Delta=0.00 \mathrm{~cm}$

$\mathrm{P}=46.43 \mathrm{~cm}$

$Q=95.73 \mathrm{~cm}$

Row $1: 8-\left(4-3 / 16^{\prime 1}\right)^{2}$ tube bars.

FIGURE III-1

4-3/16-Inch Lattice, 4-Foot Pile 


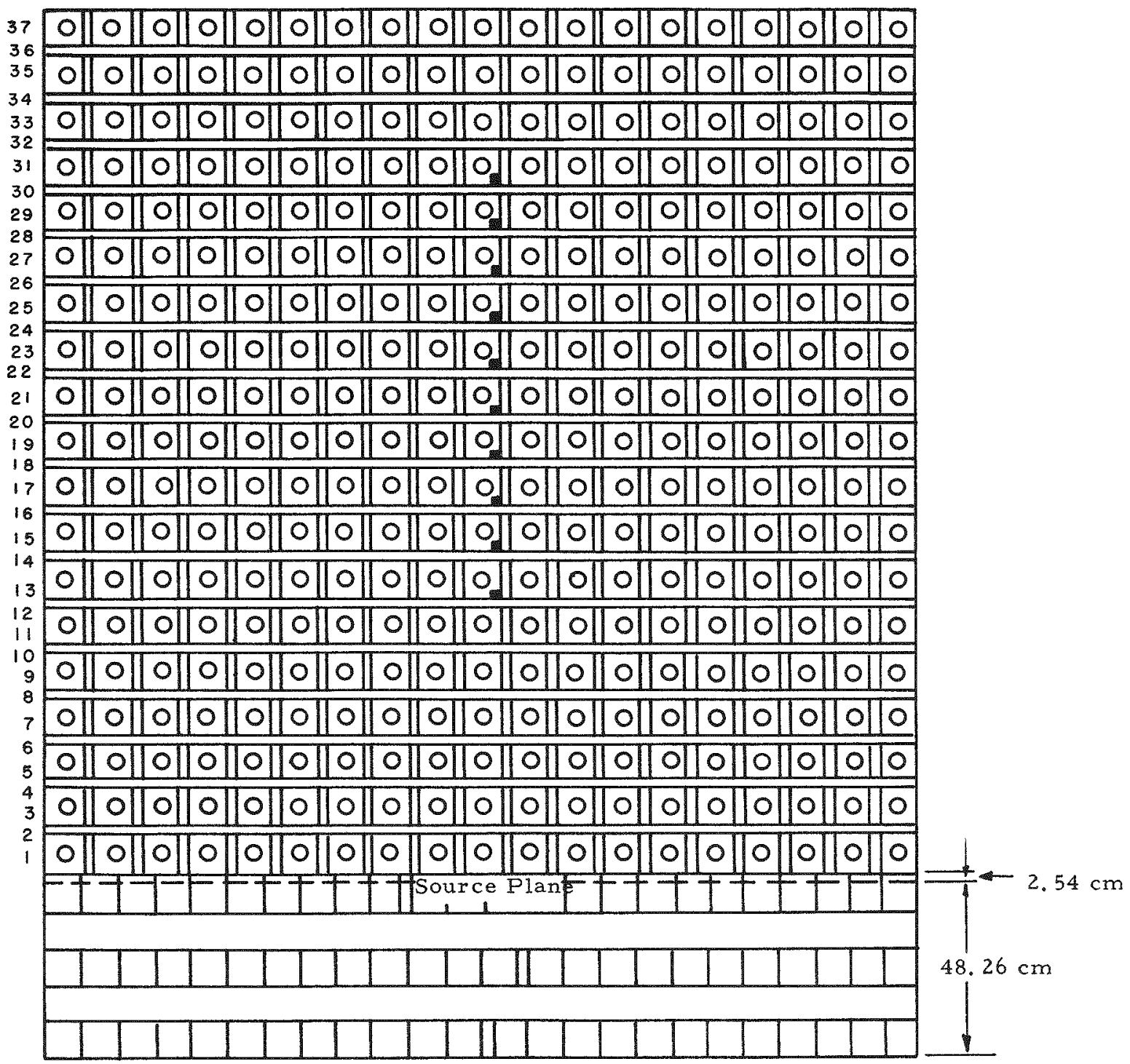

FIGURE III-2

5-3/16-Inch Lattice, 8-Foot Pile 
TABLE 2

DATA FOR 5-3/16-INCH LATTICE, 8-FOOT PILE

Fuel Elements

$0.925^{\prime \prime}$, N.U.

$1.17^{\prime \prime}, \mathrm{N} . \mathrm{U}$.

1. $36^{\prime \prime \prime}$, N.U.

1. $36^{\prime \prime \prime} \times 0.5^{i \prime}$, N.U.

$\mathrm{a}=247.809 \mathrm{~cm}$

$\mathrm{b}=243.840 \mathrm{~cm}$

$\Delta=-1.27 \mathrm{~cm}$

$\mathrm{P}=2.54 \mathrm{~cm}$

$Q=50.80 \mathrm{~cm}$

Row 1: $19-\left(4-3 / 16^{\prime \prime}\right)^{2}$ tube bars;

$$
18-\left(1^{\prime \prime} \times 4-3 / 16^{\prime \prime}\right)
$$

Row 2: $1^{\prime \prime}$ fill layer

19: Probable count bar positions

\section{FUEL ELEMENT ARRANGEMENTS}

\begin{tabular}{|c|c|}
\hline & $Z^{*}$ \\
\hline Slot & $(\mathrm{cm})$ \\
\hline 12 & 81.58 \\
\hline 14 & 94.75 \\
\hline 16 & 107.93 \\
\hline 18 & 121.11 \\
\hline 20 & 134.28 \\
\hline 22 & 147.46 \\
\hline 24 & 160.64 \\
\hline 26 & 173.81 \\
\hline 28 & 186.99 \\
\hline 30 & 200.16 \\
\hline$X=$ & $88 \mathrm{~cm}$ \\
\hline
\end{tabular}

* $Z$ distances are measured to the bottom of the count bar position.

$0.925^{\prime \prime}$ :

1. Complete loading

2. 172 tubes $0.925^{\prime \prime}$ in crimped cans buffered by $0.925^{\prime \prime}$ in $0.925^{\prime \prime} \mathrm{PT}$. $1.17^{\prime \prime}:$

1. Complete loading

$1.36^{\prime \prime} \times 0.5^{\prime \prime}$ :

1. 210 tubes of $4^{\prime \prime}$ rods through center buffered on sides by 151 tubes of $8^{\prime \prime}$ rods on sides of pile.

2. 45 tubes of $1.36^{\prime \prime} \times 0.5^{\prime \prime}$ hollow rods in core of pile with a sandwich loading. (See diagram below.)

\begin{tabular}{|c|c|c|}
\hline $\begin{array}{c}1.36^{\prime \prime} \text { solids } \\
8^{\prime \prime} \text { long }\end{array}$ & $\begin{array}{c}1.36^{\prime \prime} \text { solids } \\
4^{\prime \prime} \text { long }\end{array}$ & $\begin{array}{c}1.36^{\prime \prime} \text { solids } \\
8^{\prime \prime} \text { long }\end{array}$ \\
\hline $\begin{array}{c}1.36^{\prime \prime} \text { solids } \\
\text { with } 0.04^{\prime \prime} \\
\text { spacers }\end{array}$ & $\begin{array}{c}1.36^{\prime \prime} \times 0.5^{\prime \prime} \\
\text { hollow rods }\end{array}$ & $\begin{array}{c}1.36^{\prime \prime} \text { solids } \\
\text { with } 0.04^{\prime \prime} \\
\text { spacers }\end{array}$ \\
\hline $\begin{array}{c}1.36^{\prime \prime} \text { solids } \\
8^{\prime \prime} \text { long }\end{array}$ & $\begin{array}{c}1.36^{\prime \prime} \text { solid } \\
4^{\prime \prime} \text { long }\end{array}$ & $\begin{array}{c}1.36^{\prime \prime} \text { solids } \\
8^{\prime \prime} \text { long }\end{array}$ \\
\hline
\end{tabular}




\section{TABLE 2 (contd)}

3. 24 tubes $1.34^{\prime \prime} \times 0.5^{\prime \prime}$ hollow rods in core of pile in sandwich loading. (See diagram below.)

\begin{tabular}{|c|c|c|c|c|}
\hline $\begin{array}{l}\text { 1. } 36^{\prime \prime} \text { solids } \\
8^{\prime \prime} \text { long }\end{array}$ & \multicolumn{3}{|c|}{$\begin{array}{c}1.36^{\prime \prime} \text { solids } \\
4^{\prime \prime} \text { long }\end{array}$} & $\begin{array}{c}.36^{\prime \prime} \text { solids } \\
8 " \text { long }\end{array}$ \\
\hline $\begin{array}{c}1.36^{\prime \prime} \times 0.5^{\prime \prime} \\
\text { hollow }\end{array}$ & $\begin{array}{l}1.36^{\prime \prime} \\
\text { solids } \\
4^{\prime \prime} \text { long }\end{array}$ & $\begin{array}{c}1.36^{\prime \prime} \times 0.5^{m} \\
\text { hollow }\end{array}$ & $\begin{array}{l}1.36^{\prime \prime} \\
\text { solids } \\
4^{\prime \prime} \text { long }\end{array}$ & $\begin{array}{c}1.36^{\prime \prime} \times 0.5^{\prime \prime} \\
\text { hollow }\end{array}$ \\
\hline $\begin{array}{c}\text { 1. } 36^{\prime \prime} \text { solids } \\
8^{\prime \prime} \text { long }\end{array}$ & \multicolumn{3}{|c|}{$\begin{array}{c}1.36^{\prime \prime} \text { solids } \\
4^{\prime \prime} \text { long }\end{array}$} & $\begin{array}{c}1.3 \overline{6}^{\prime \prime} \text { solids } \\
8^{\prime \prime} \text { long }\end{array}$ \\
\hline
\end{tabular}

$1.36^{\prime \prime}$

1. 184 tubes of 4 " long rods through center of pile, with 151 tubes of 8 " long rods buffering the sides.

2. 210 tubes of $4^{\prime \prime}$ solid rods with 151 tubes of 8 " rods for buffer region on sides.

The complex core loadings were used due to lack of sufficient fuel for a complete load. Different loadings were used to evaluate the precision of the results for partial loadings. 
Fuel Elements:

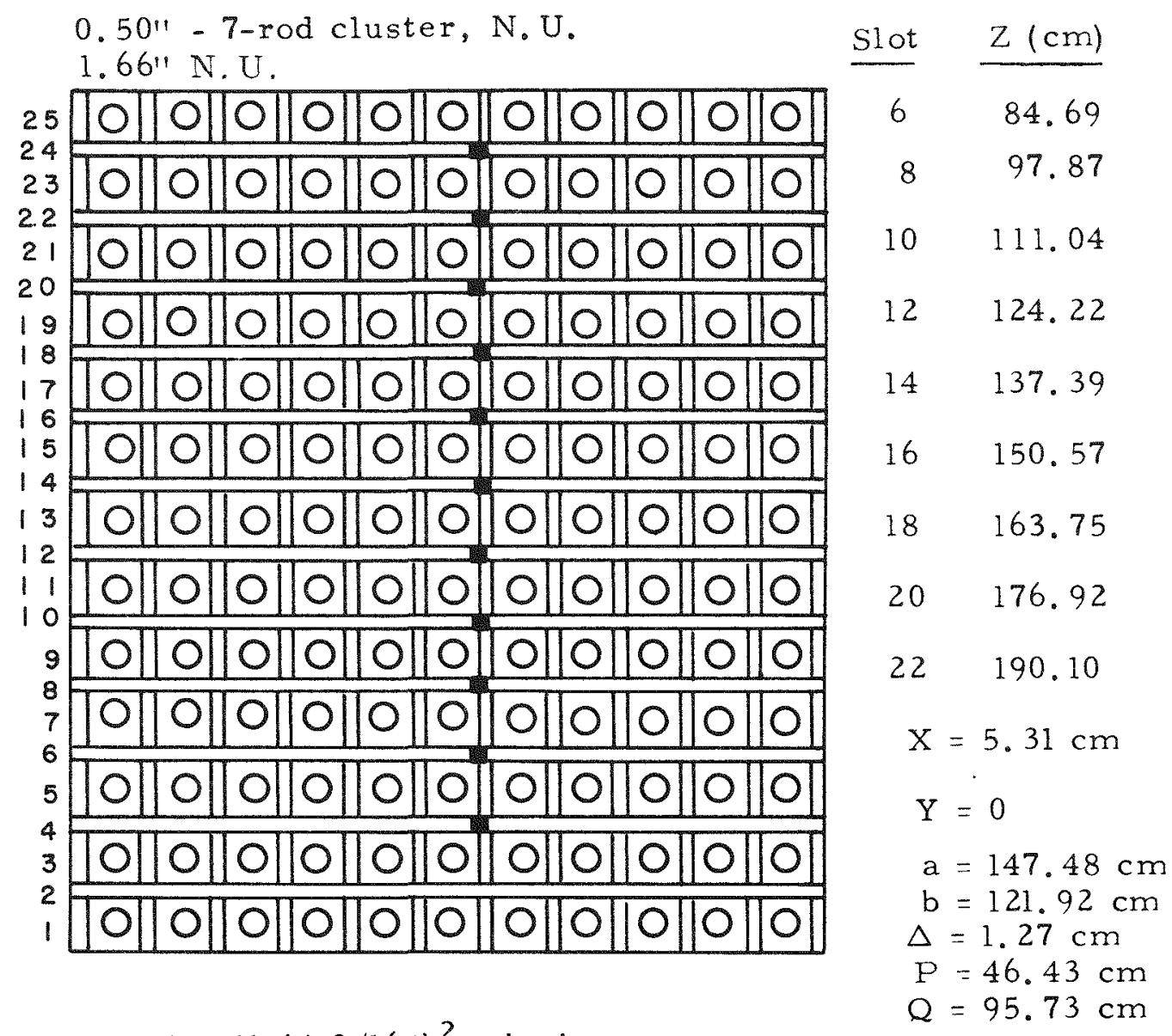

Row 1: $\quad 11-\left(4-3 / 16^{\prime \prime}\right)^{2}$ tube bars, $12-(1 " \times 4-3 / 16 ")$

Row 2: I" fill layer

FIGURE III-3

5-3/16-Inch Lattice, 4-Foot Pile 


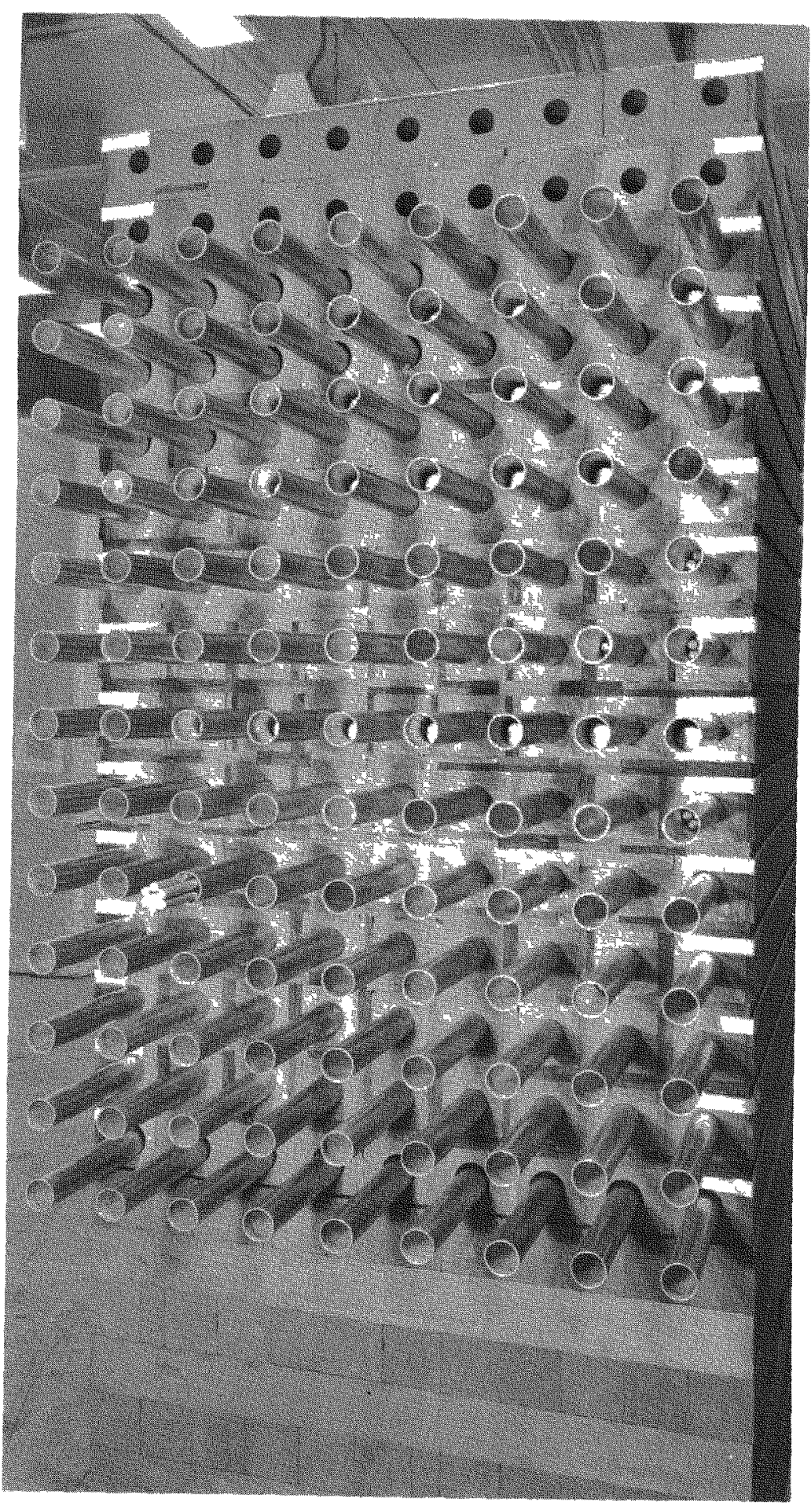

FIGURE III-3A

5-3/16-Inch Lattice, 4-Foot Pile

$0581089-4$ 0.5-Inch Rod Clusters 
Fuel Elements:

*1. $343 "$ - $7.5 \%$ U-Al Alloy

1. 343" - 4.46\% U-Al Alloy

1. $36^{\prime \prime}-0.94 \%$ Enrichment

1. $66^{\prime \prime}-1.007 \%$ Enrichment

1. $37 " 1 \times 0.48 " 1$ - $1.44 \%$ Enrichment

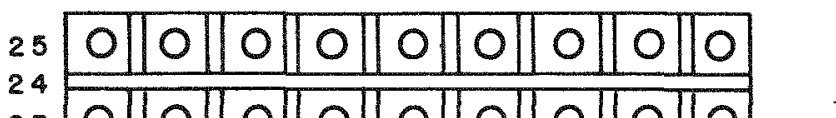

23

22

21

20

19

18

16

15

14

13

12

11

10

9

7

6

4

3

2

1

$a=116.05 \mathrm{~cm}$ $\mathrm{b}=121.92 \mathrm{~cm}$ $\Delta=-1.27 \mathrm{~cm}$ $\mathrm{P}=46.43 \mathrm{~cm}$

$\mathrm{Q}=95.73 \mathrm{~cm}$

*Also used upper source position: $P=3.89 \mathrm{~cm}$

Row 2: 1" fill layer

\begin{tabular}{|c|c|}
\hline Slot & $Z(\mathrm{~cm})$ \\
\hline 4 & 71.51 \\
\hline 6 & 84.69 \\
\hline 8 & 97.87 \\
\hline 10 & 111.04 \\
\hline 12 & 124.22 \\
\hline 14 & 137.39 \\
\hline 16 & 150.57 \\
\hline 18 & 163.75 \\
\hline 20 & 176.92 \\
\hline 22 & 190.10 \\
\hline & U \\
\hline Y & \\
\hline
\end{tabular}

Subtract $42.54 \mathrm{~cm}$ for $Z$ values with upper source position.

tube bars; $8-\left(1^{\prime \prime} \times 4-3 / 16^{\prime \prime}\right)$

FIGURE III-4

5-3/16-Inch Lattice, 4-Foot Pile 
Fuel Elements (All N. U.):

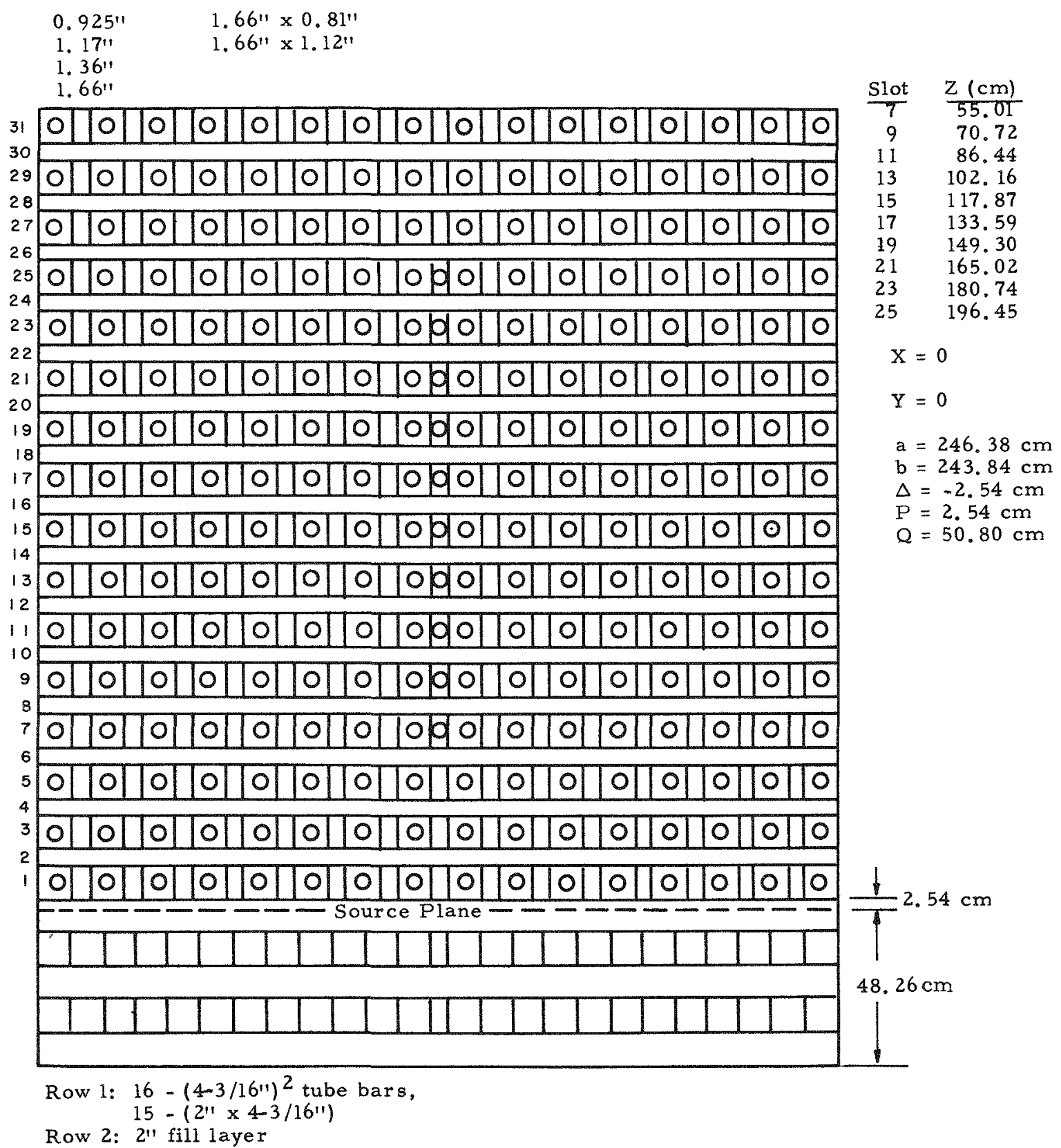

Outside edge of $0.925^{\prime \prime}$ solid lattice had $0.925^{\prime \prime}$ rods in different aluminum cans.

FIGURE III - 5

6-3/16-Inch Lattice, 8-Foot Pile 
Fuel Elements:

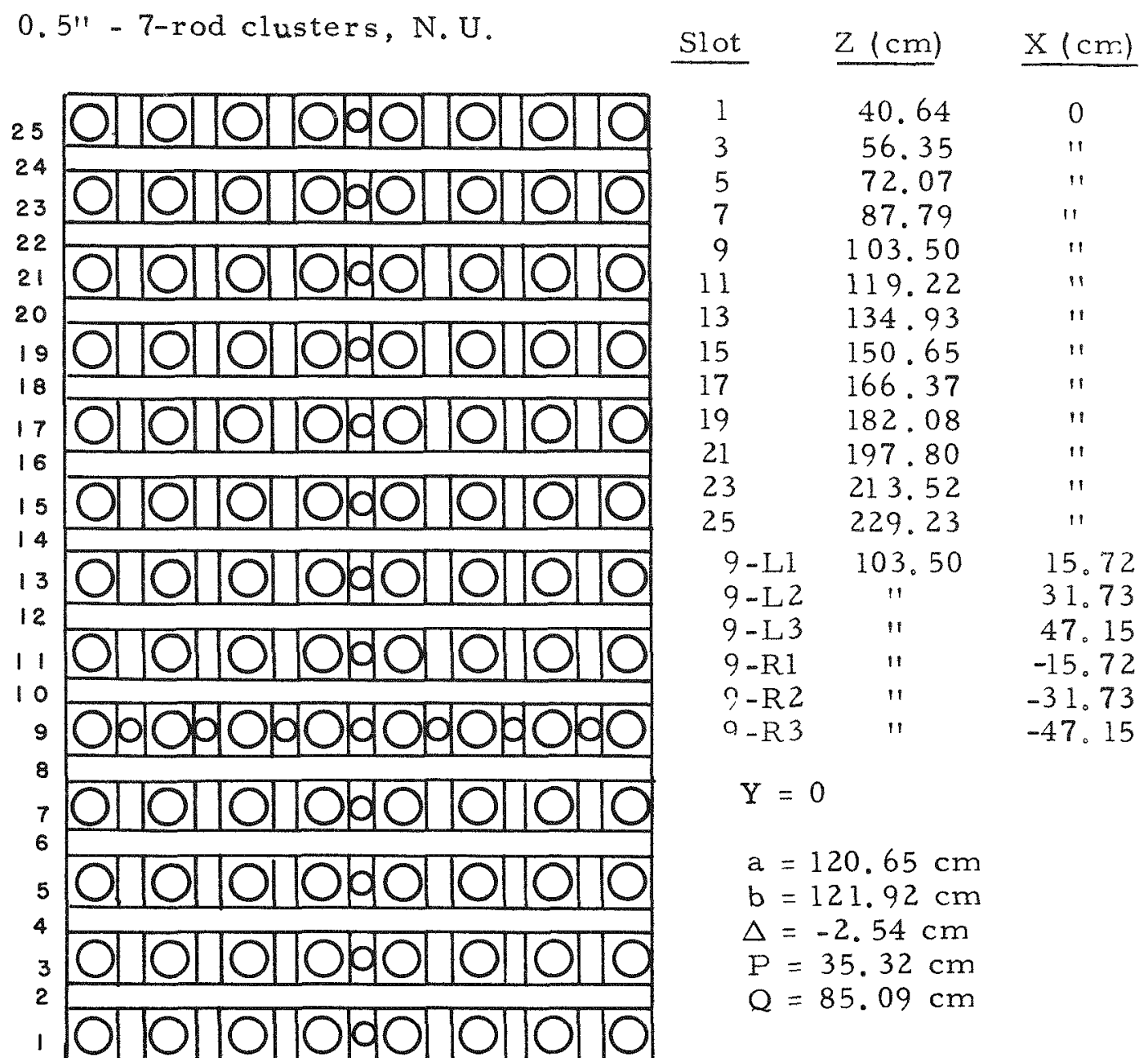

Row 1: $8-\left(4-3 / 16^{\prime \prime}\right)^{2}$ tube bars;

6-(2"1 x 4-3/16"1);

$1-\left(2^{\prime \prime} \times 4-3 / 16^{\prime \prime}\right)$ count bar

Row 2: 8-(2"' x 4-3/16"');

$4-\left(3-1 / 2^{\prime \prime} \times 2^{\prime \prime}\right)$;

Row 9: $8-(4-3 / 16 " 1)^{2}$ tube bars;

$7-\left(2^{\prime \prime} \times 4-3 / 16^{\prime \prime}\right)$ count bars.

For the $1.92^{\prime \prime}$ fuel, the top two layers contained 1.66 " solid fuel.

FIGURE III-6

6-3/16-Inch Lattice, 4-Foot Pile 
Fuel Elements:

$0.925^{\prime \prime}-1.007 \%$ Enrichment

$1.17 "$ N.U.

$1.343^{\prime \prime}-4.46 \%$ U-Al Alloy

1. $343^{\prime \prime}-7.5 \% \mathrm{U}-\mathrm{Al}$ Alloy

$1.36^{\prime \prime} \mathrm{N} . \mathrm{U}$.

1.336" - 0.94\% Enrichment

$1.66 " 1$ - $1.007 \%$ Enrichment

$1.37 " 1 \times 0.48^{\prime \prime}-1.44 \%$ Enrichment Slot $\mathrm{Z}(\mathrm{cm})$
$1.66^{\prime \prime} \times 0.94^{\prime \prime}-1.007 \%$ Enrichment

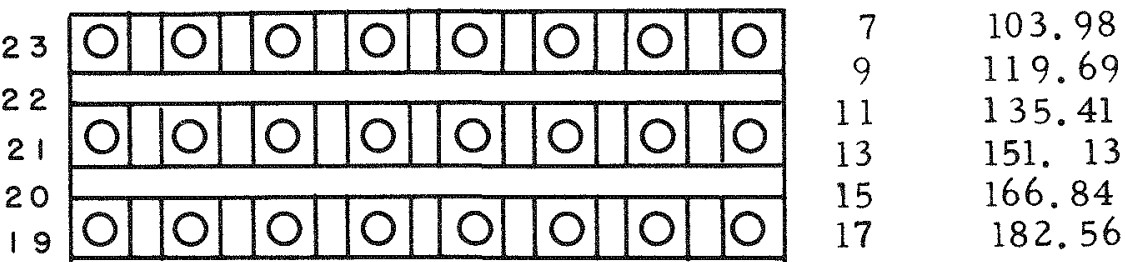

18

17

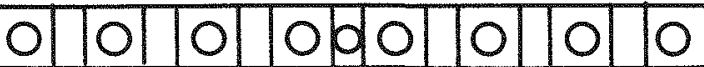

16

15

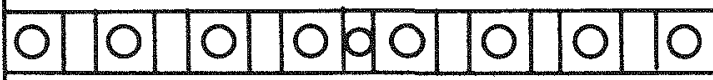

14

13

-

12

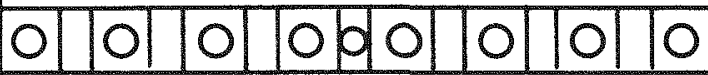

11

(

-

8

o lollollodollollo

O|lO|lollollollollollo

4

3

2

1

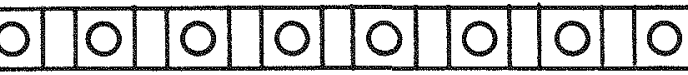

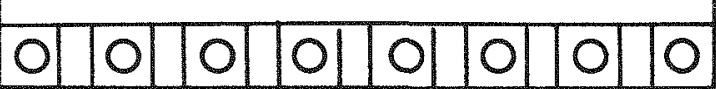

$X=0$

$\mathrm{Y}=0$

$a=121.92 \mathrm{~cm}$

$b=121.06 \mathrm{~cm}$

$\Delta=-2.54 \mathrm{~cm}$

$P=46.43 \mathrm{~cm}$

$Q=95.73 \mathrm{~cm}$

Row 1: $8-\left(4-3 / 16^{(1)}\right)^{2}$ tube bars;

$7-\left(2^{\prime \prime} \times 4=3 / 16^{\prime \prime}\right)$

Row 2: 2 "fill layer

FIGURE III-7

6-3/16-Inch Lattice, 4-Foot Pile 
Fuel Elements:

$1.66^{11}-1.007 \%$ Enrichment

$0.925^{\prime \prime}-1.007 \%$ Enrichment

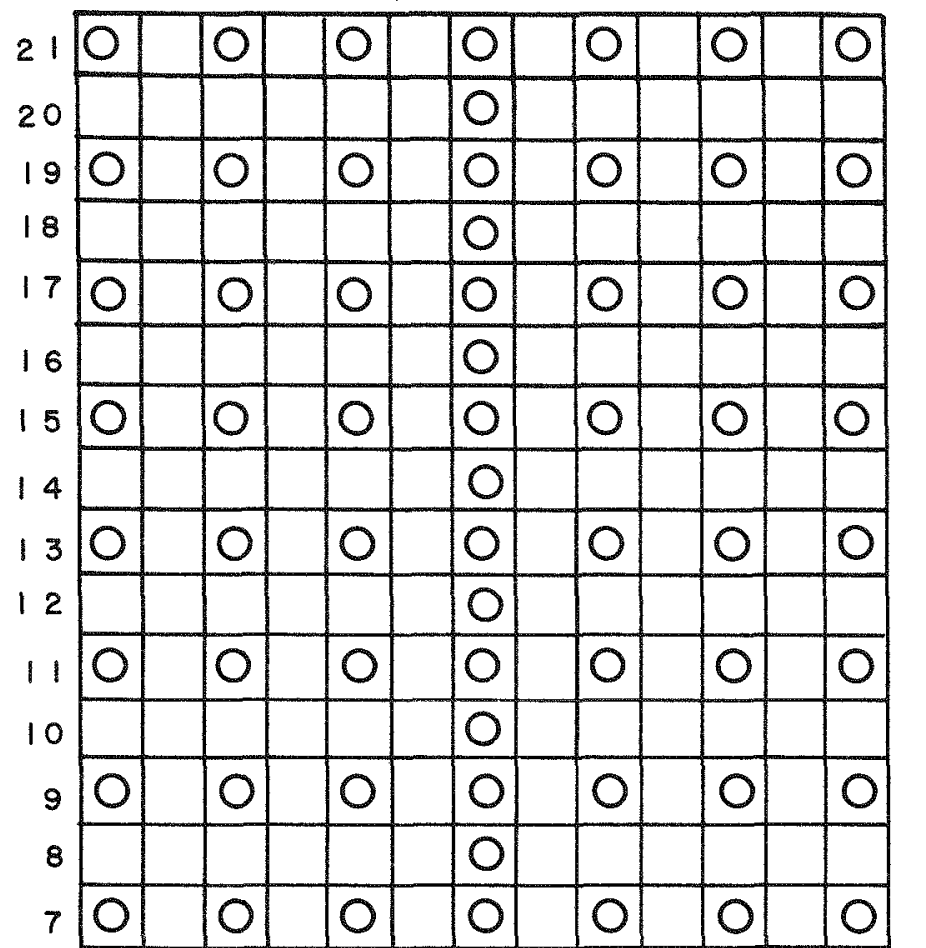

$\begin{array}{ccc}\text { Slot } & & \frac{Z(\mathrm{~cm})}{4} \\ 4 & & 77.07 \\ 6 & & 94.85 \\ 8 & & 112.63 \\ 10 & & 130.41 \\ 12 & & 148.19 \\ 14 & & 165.97 \\ 16 & & 183.75 \\ 18 & 201.53 \\ 20 & 219.31\end{array}$

$X=0$

$Y=0$

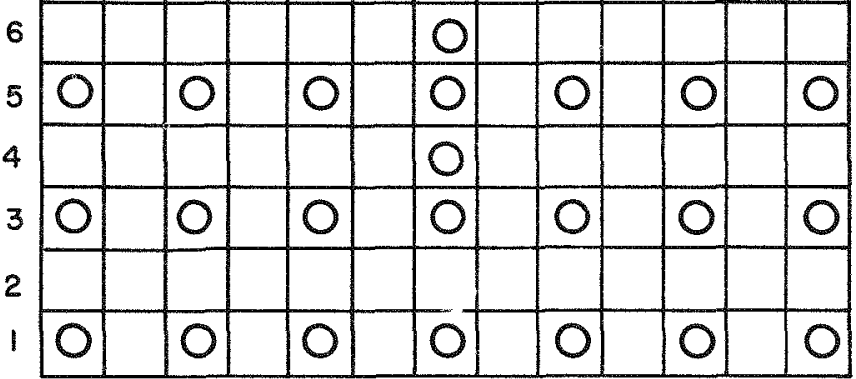

$\mathrm{a}=115.57 \mathrm{~cm}$

$\mathrm{b}=121.92 \mathrm{~cm}$

$\Delta=-4.44 \mathrm{~cm}$

$\mathrm{P}=45.96 \mathrm{~cm}$

$Q=106.36 \mathrm{~cm}$

Row 1: $7-\left(3-1 / 2^{\prime \prime}\right)^{2}$ tube bars;

$6-\left(3-1 / 2^{\prime \prime}\right)^{2}$

Row 4: $12-\left(3-1 / 2^{\prime \prime}\right)^{2}$

$1-(3-1 / 2 " 1)^{2}$ count bar

FIGURE III-8

7-Inch Lattice, 4-Foot Pile 


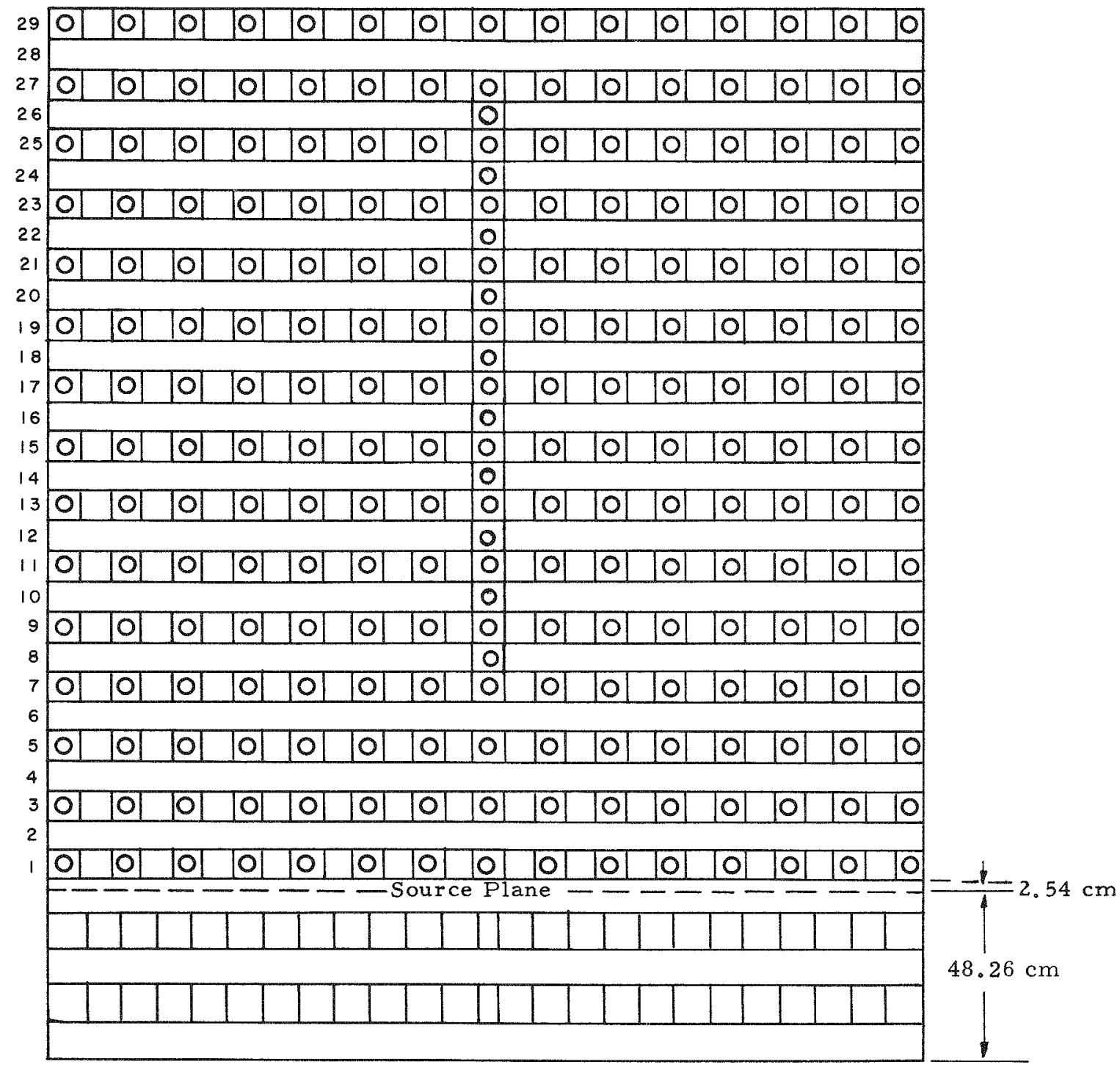

FIGURE III-9

7-Inch Lattice, 8-Foot Pile 
HW -69525

TABLE 3

DATA FOR 7-INCH LATTICE, 8-FOOT PILE

Fuel Elements:

$0.925^{\prime \prime}$, N.U.

$1.17^{\prime \prime}$, N.U.

$1.36^{\prime \prime}$, N.U.

$1.36^{\prime \prime} \times 0.50^{\prime \prime}, \mathrm{N} . \mathrm{U}$.

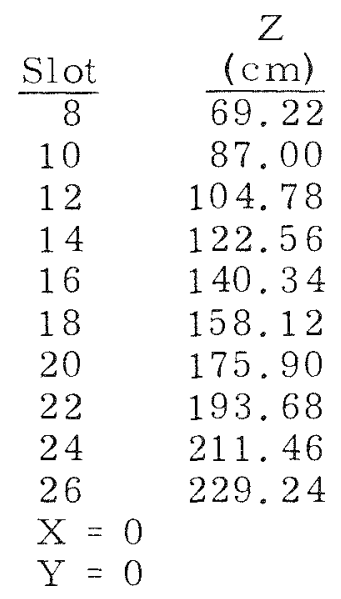

Row 1: $15-\left(3-1 / 2^{\prime \prime}\right)^{2}$ tube bars;

$\mathrm{a}=257.81 \mathrm{~cm}$

$\mathrm{b}=243.84 \mathrm{~cm}$

$\Delta=-4.44 \mathrm{~cm}$

$\mathrm{P}=254 \mathrm{~cm}$

$\mathrm{Q}=50.80 \mathrm{~cm}$

$$
14-\left(3-1 / 2^{\prime \prime}\right)^{2}
$$

Row 2: $29-\left(3-1 / 2^{\prime \prime}\right)^{2}$

\section{FUEL ELEMENT ARRANGEMENTS}

$0.925^{\prime \prime}:$

1. Complete loading

2. 172 tubes $0.925^{\prime \prime}$ rods in crimped cans buffered by 53 tubes of $0.925^{\prime \prime}$ rods in $0.925 \mathrm{PT}$

$1.17^{11}:$

1. Complete loading

$1.38^{\prime \prime}:$

1. Complete loading of $1.36^{\prime \prime}$ rods, 4 " long.

2. 173 tubes 1.36 " rods, 4" long buffered by 1.36 " rods 8 " long on sides of pile.

3. 111tubes $1.36^{\prime \prime}$ rods, 4 "long, through center of pile buffered by 114 tubes $1.36^{\prime \prime}$ rods, 8 "long, on sides of pile.

$1.36^{\prime \prime} \times 0.50^{\prime \prime}:$

1. 111 tubes $1.36^{\prime \prime} \times 0.50^{\prime \prime}$ rods, $4^{\prime \prime}$ long, buffered on sides of pile by 1.36 " 10.50 " rods, 8 " long.

2. 45 tubes $1.36^{\prime \prime} \times 0.50^{\prime \prime}$ rods, 4 " long, buffered by 108 tubes of $1.36^{\prime \prime} \times 0.50^{\prime \prime}$ rods, 8 "long and 70 tubes of $1.36^{\prime \prime}$ rods, 4 "l long. 
Fuel Element:

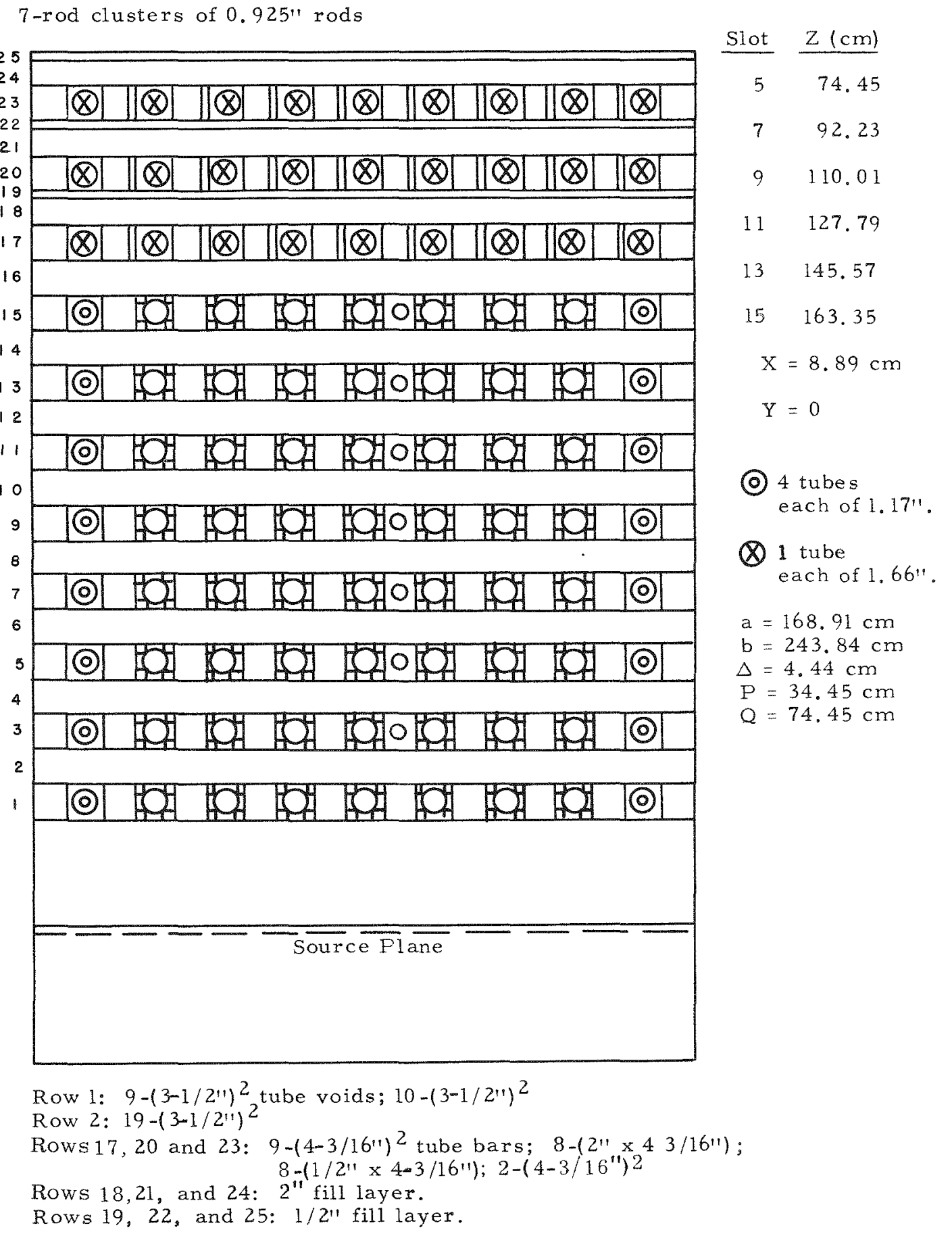

FIGURE IIT-10

7-Inch Lattice, 8-Foot Pile 


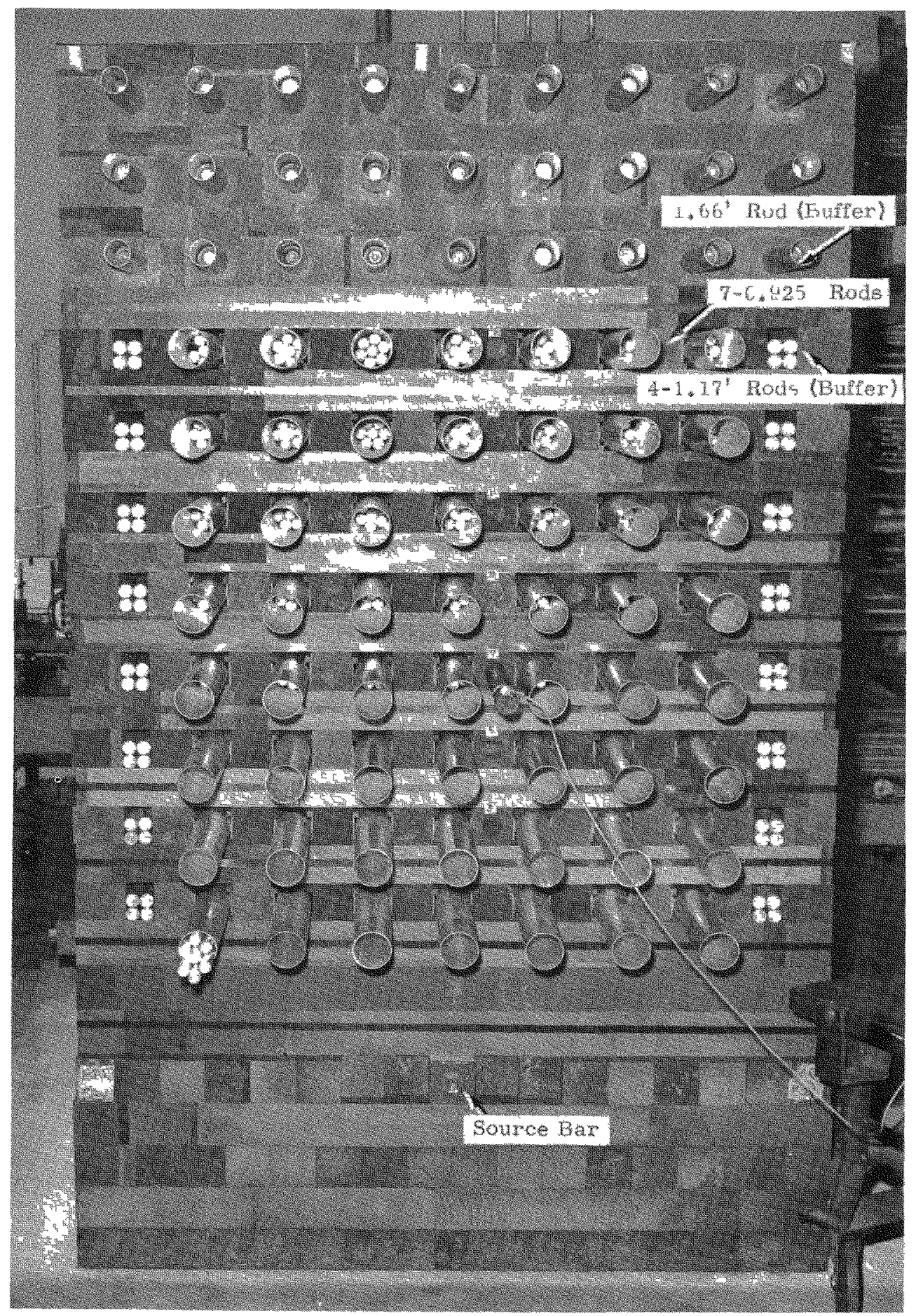

FIGURE III-10A

7-Inch Lattice, 8-Foot Pile

0571424-2

0.925 -Inch Rod Clusters 
Fuel Elements:

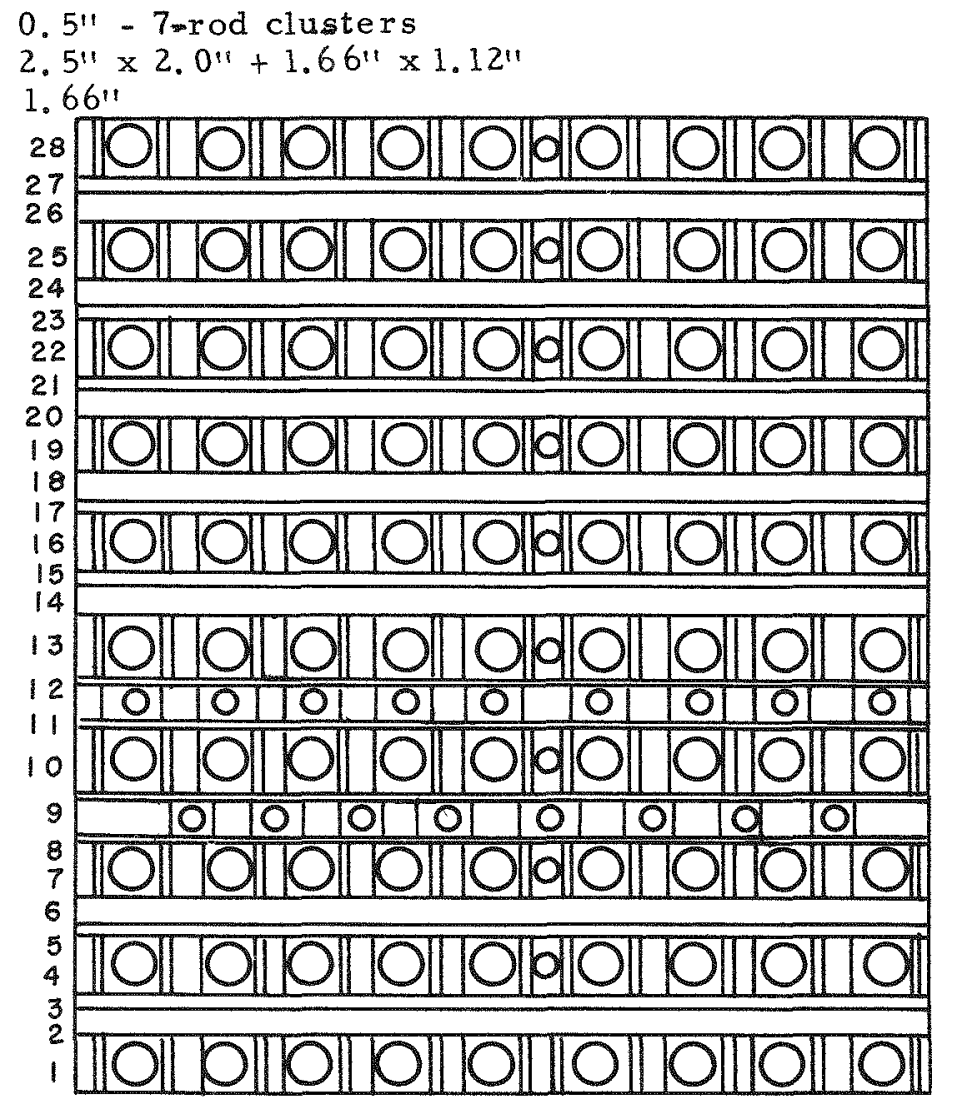

Row 1: $9-\left(4-3 / 16^{\prime \prime}\right)^{2}$ tube bars; $8-\left(2^{\prime \prime} \times 4-3 / 16 " 1\right)$; $9-\left(1^{\prime \prime} \times 4-3 / 16^{\prime \prime}\right) ; 4-\left(1 / 2^{\prime \prime} \times 4-3 / 16^{\prime \prime}\right)$

Row 2: $13-\left(2^{\prime \prime} \times 4-3 / 16^{\prime \prime}\right) ; 12-\left(1 / 2^{\prime \prime} \times 3^{\prime \prime}\right)$

Row 3: $13-\left(1 " 1 \times 4-3 / 16^{\prime \prime}\right) ; 6-\left(1 / 2^{\prime \prime} \times 33^{\prime \prime}\right)$

Row 9: $8-\left(2^{\prime \prime} \times 4-3 / 16 "\right)$ count bars; $7-(2 " 1 \times 3 ")$; $2-\left(2^{\prime \prime} \times 4-3 / 16^{\prime \prime}\right)$

Row 10: 9-(4=3/16"' ${ }^{2}$ tube bars; $8-\left(2^{\prime \prime} \times 4-3 / 16^{\prime \prime}\right)$ count bars; $18-\left(1 / 2^{\prime \prime} \times 4-3 / 16^{\prime \prime}\right)$; $2-(1 " \times 4=3 / 16 ")$

Rows 8 and 11: $1 / 2^{\prime \prime}$ fill layers

Row 12: 9-(2" $\left.\times 4-3 / 16^{\prime \prime}\right)$ count bars; $8-\left(2^{\prime \prime} \times 3 " 1\right)$; $2-(1=1 / 2 " \times 2 " 1)$

\begin{tabular}{|c|c|c|}
\hline & & \\
\hline$\frac{\text { Slot }}{4}$ & $\frac{\mathrm{Z} *(\mathrm{~cm})}{69.53}$ & $\frac{X(\mathrm{~cm})}{9.13}$ \\
\hline 7 & 87.78 & iI \\
\hline 10 & 106.04 & $"$ \\
\hline 13 & 124.30 & $"$ \\
\hline 16 & 142.55 & $"$ \\
\hline 19 & 160.81 & $"$ \\
\hline 22 & 179.07 & $"$ \\
\hline 25 & 197.32 & 11 \\
\hline 28 & 215.58 & 11 \\
\hline 9 & 96.91 & 0.0 \\
\hline $9-\mathrm{LI}$ & $"$ & 9.13 \\
\hline $9-L 2$ & $" 1$ & 27.38 \\
\hline $9-L 3$ & 11 & 45.64 \\
\hline $9-\mathrm{L} 4$ & $"$ & 63.90 \\
\hline 12 & 115.17 & 0.0 \\
\hline $12-\mathrm{L} 1$ & $"$ & 18.26 \\
\hline $12-\mathrm{L} 2$ & $"$ & 36.51 \\
\hline $12-\mathrm{L} 3$ & $"$ & 54.77 \\
\hline $12-\mathrm{L} 4$ & $"$ & 73.02 \\
\hline $\mathrm{Y}$ & & \\
\hline & $164.41 \mathrm{~cm}$ & \\
\hline & $121.92 \mathrm{~cm}$ & \\
\hline$\Delta$ & $0.00 \mathrm{~cm}$ & \\
\hline & $95.73 \mathrm{~cm}$ & \\
\hline & $\begin{array}{l}48.14 \text { for } \\
\text { in -tube. }\end{array}$ & he tube - \\
\hline & $\begin{array}{l}45.96 \text { for } \\
\text { and } 1.66 " 1\end{array}$ & $\begin{array}{l}\text { clusters } \\
\text { solid }\end{array}$ \\
\hline & $7-\operatorname{rod} c 1$ & isters and \\
\hline & 6" solid & $\mathrm{dd} 2.18 \mathrm{~cm}$ \\
\hline & $\begin{array}{l}\text { yet the c } \\
\text { alues fo }\end{array}$ & $\begin{array}{l}\text { responding } \\
\text { he tube-in }\end{array}$ \\
\hline
\end{tabular}

The tube-in-tube lattice was lacking Rows 27 and 28.

FIGURE III-11

7-3/16-Inch Lattice, 4-Foot Pile 


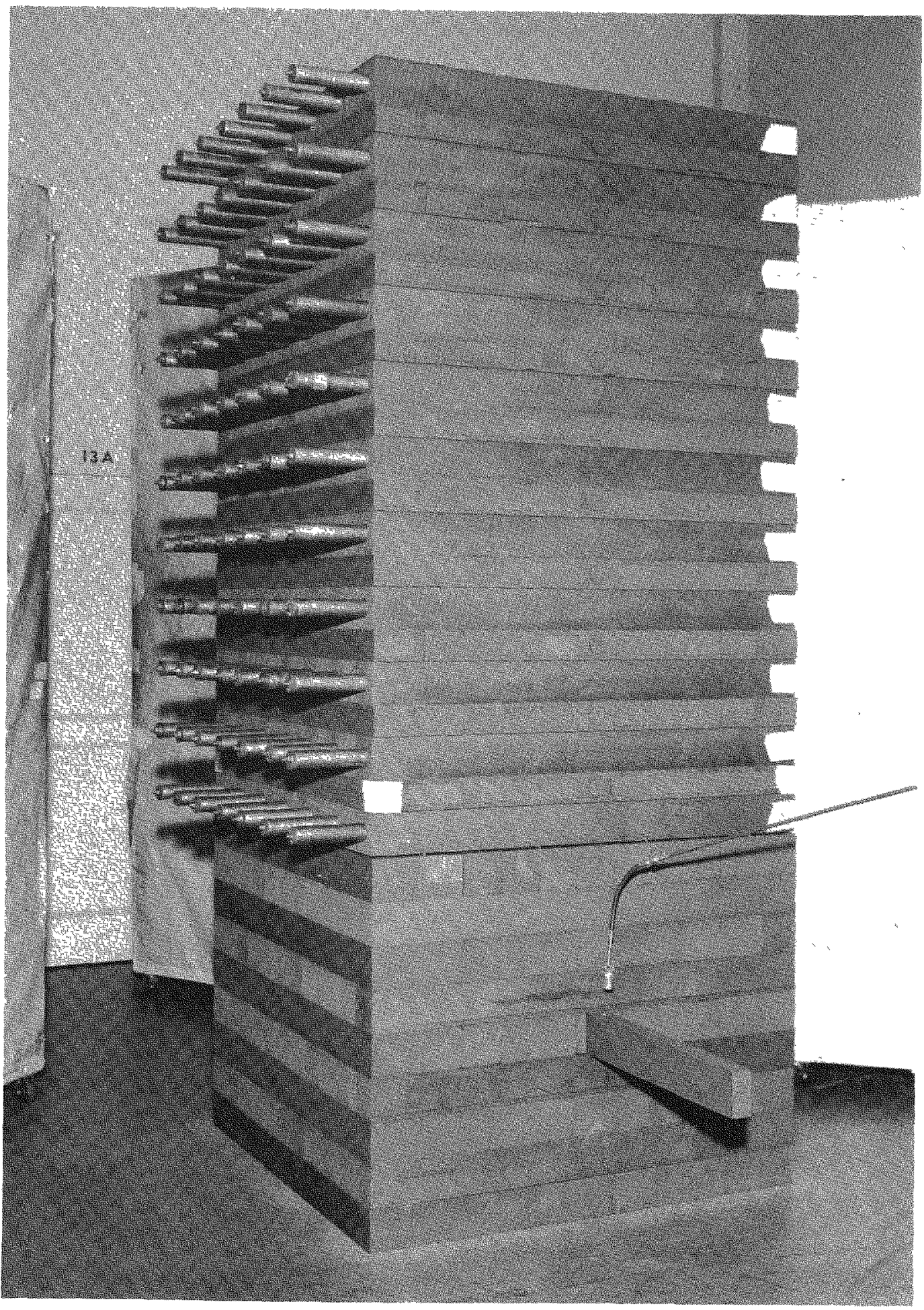

FIGURE III-11A

7-3/16-Inch Lattice, 4-Foot Pile $12683-2$

1.66-Inch Solid Fuel 


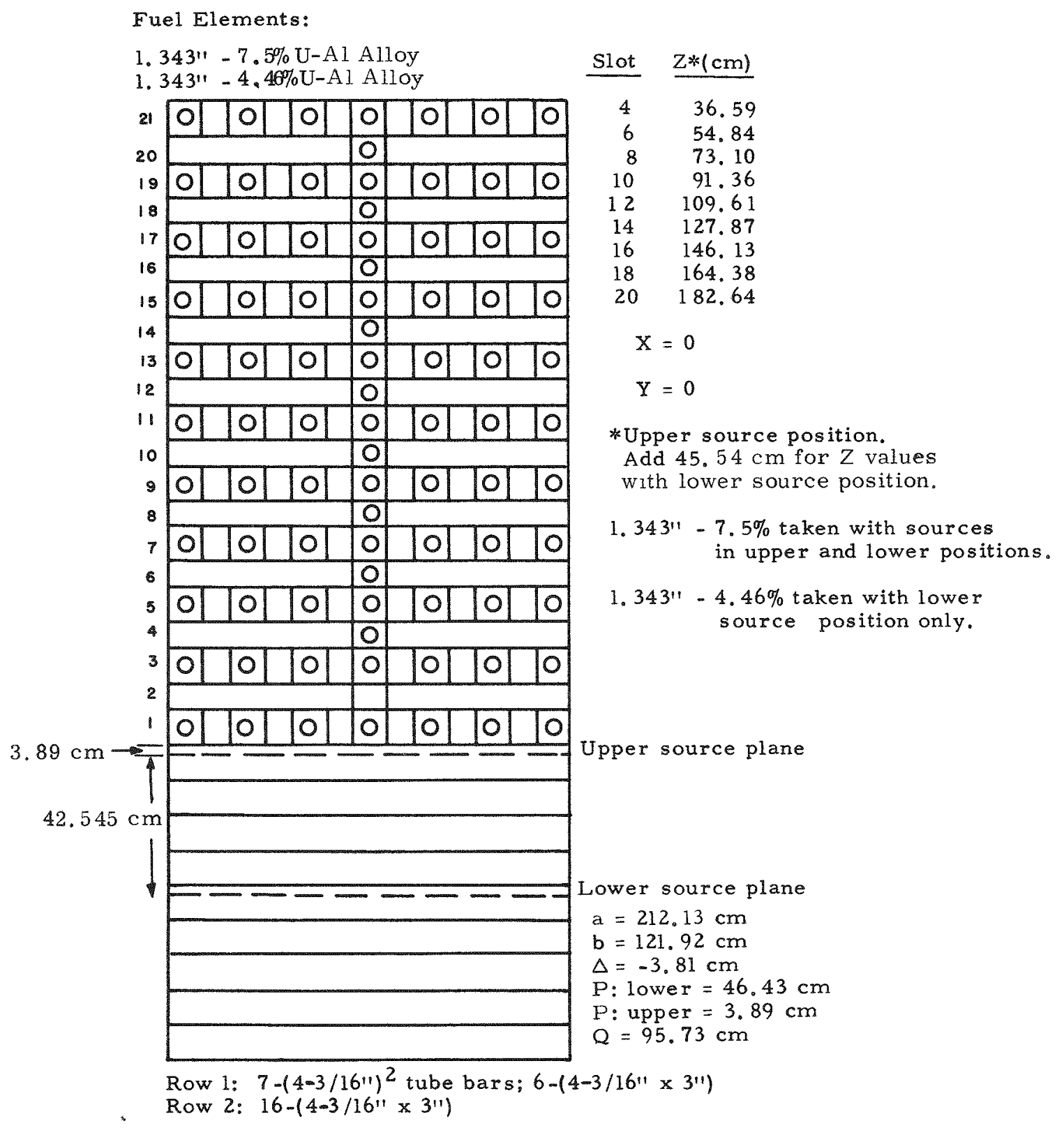

FIGURE III-12

7-3/16-Inch Lattice, 4-Foot Pile 


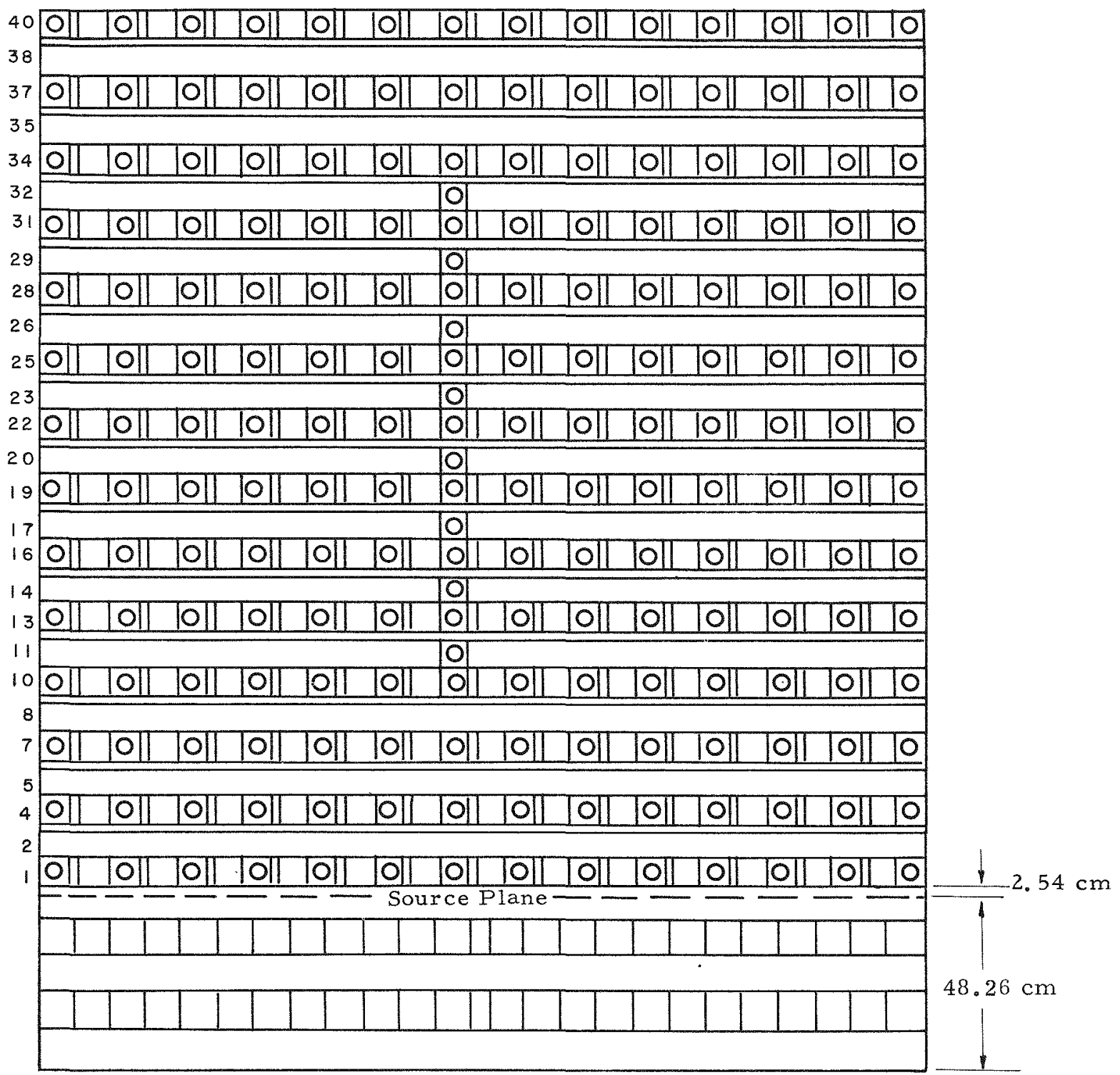

FIGURE III -13

7-1/2-Inch Lattice, 8-Foot Pile 


\section{TABLE 4}

\section{DATA FOR 7-1/2-INCH LATTICE, 8-FOOT PILE}

Fuel Elements:

$0.925 "$, N.U.

1. $17^{\prime \prime}$, N.U.

1. $36^{\prime \prime \prime}$, N.U.

1. $66^{\prime \prime \prime}$, N.U.

$1.66^{\prime \prime} \times 0.81^{\prime \prime}$, N.U.

$1.66^{\prime \prime} \times 1.12^{\prime \prime}$, N.U.

$\begin{array}{lll}\text { Slot } & \frac{Z}{(\mathrm{~cm})} \\ 11 & \frac{73.02}{14} & 92.08 \\ 17 & 111.12 \\ 20 & 130.18 \\ 23 & 149.22 \\ 26 & 168.28 \\ 29 & 187.32 \\ 32 & 206.38 \\ Y= & -6.35 \mathrm{~cm} \text { for the } 1.17^{\prime \prime} \mathrm{rod} \\ Y= & 0 \text { for all others } \\ X= & -9.52 \mathrm{~cm}\end{array}$

$\mathrm{a}=256.54 \mathrm{~cm}$

$\mathrm{b}=243.84 \mathrm{~cm}$

$\Delta=-5.08 \mathrm{~cm}$

$\mathrm{P}=2.54 \mathrm{~cm}$

$Q=50.80 \mathrm{~cm}$

Row 1: $14-\left(3-1 / 2^{\prime \prime}\right)^{2}$ tube bars;

$13-\left(3-1 / 2^{\prime \prime}\right)^{2}$

$13-\left(1 / 2^{\prime \prime} \times 3-1 / 2^{\prime \prime}\right)$

Row 2: $3-1 / 2^{\prime \prime}$ fill layer

Row 3: $1 / 2^{\prime \prime}$ fill layer 
Fuel Elements:

$0.925^{\prime \prime}$ N.U.

$0.925^{\prime \prime}-1.007 \%$ Enrichment

$1.36 " 1 \mathrm{~N} . \mathrm{U}$.

$1.66^{\prime \prime}-1.007 \%$ Enrichment

$1.66^{\prime \prime} \times 0.94^{\prime \prime}-1.007 \%$ Enrichment

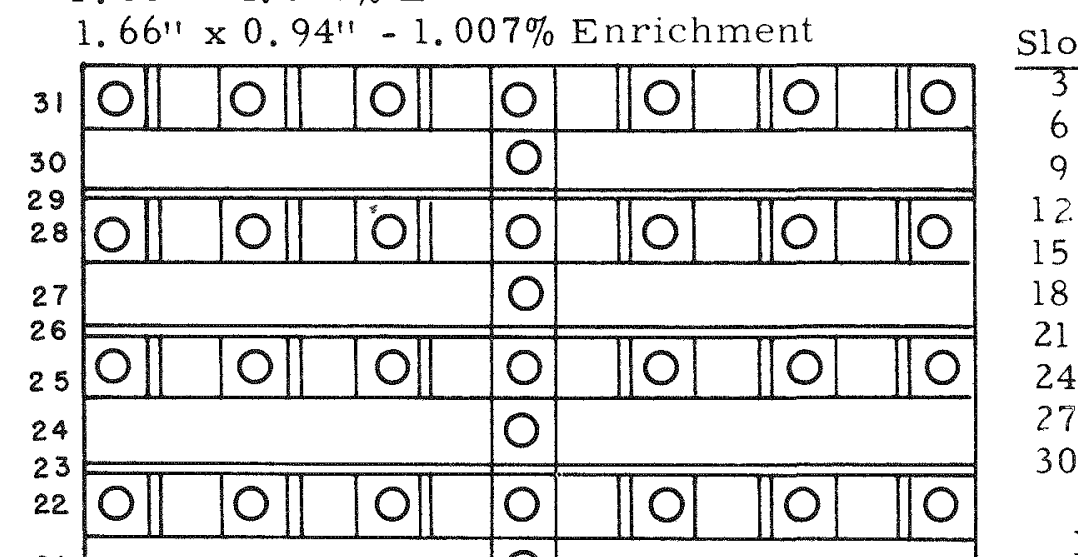

$\frac{\text { Slot }}{3} \quad \frac{Z(\mathrm{~cm})}{60.56}$

$6 \quad 79.61$

998.66

12. 117.71

$15 \quad 136.76$

$18 \quad 155.81$

$21 \quad 174.86$

$24 \quad 193.91$

$27 \quad 212.96$

$30 \quad 232.01$

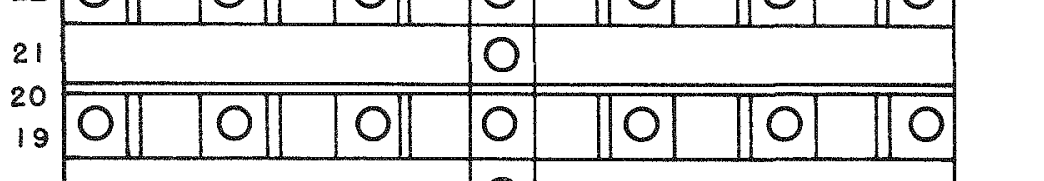

$x=0$

$Y=0$

$a=123.19 \mathrm{~cm}$

$\mathrm{b}=121.92 \mathrm{~cm}$

$\Delta=-5.08 \mathrm{~cm}$

$\mathrm{P}=45.96 \mathrm{~cm}$

$Q=95.73 \mathrm{~cm}$

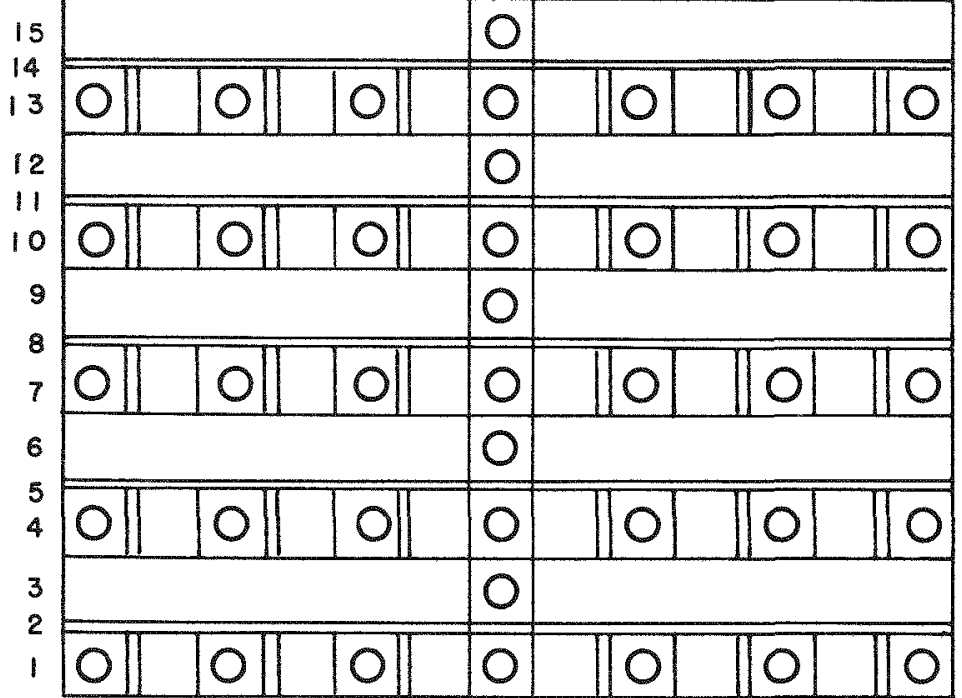

Row 1: $7-\left(3-1 / 2^{\prime \prime}\right)^{2}$ tube bars; $6-\left(3-1 / 2^{\prime \prime}\right)^{2}$; $6-\left(1 / 2^{\prime \prime} \times 3-1 / 2^{\prime \prime}\right)$

Row 2: $1 / 2$ " fill layer

Row 3: $1-\left(3-1 / 2^{\prime \prime}\right)^{2}$ count bar; $15-\left(3-1 / 2^{\prime \prime}\right)^{2}$

FIGURE III-14

7-1/2-Inch Lattice, 4-Foot Pile 
Fuel Elements:

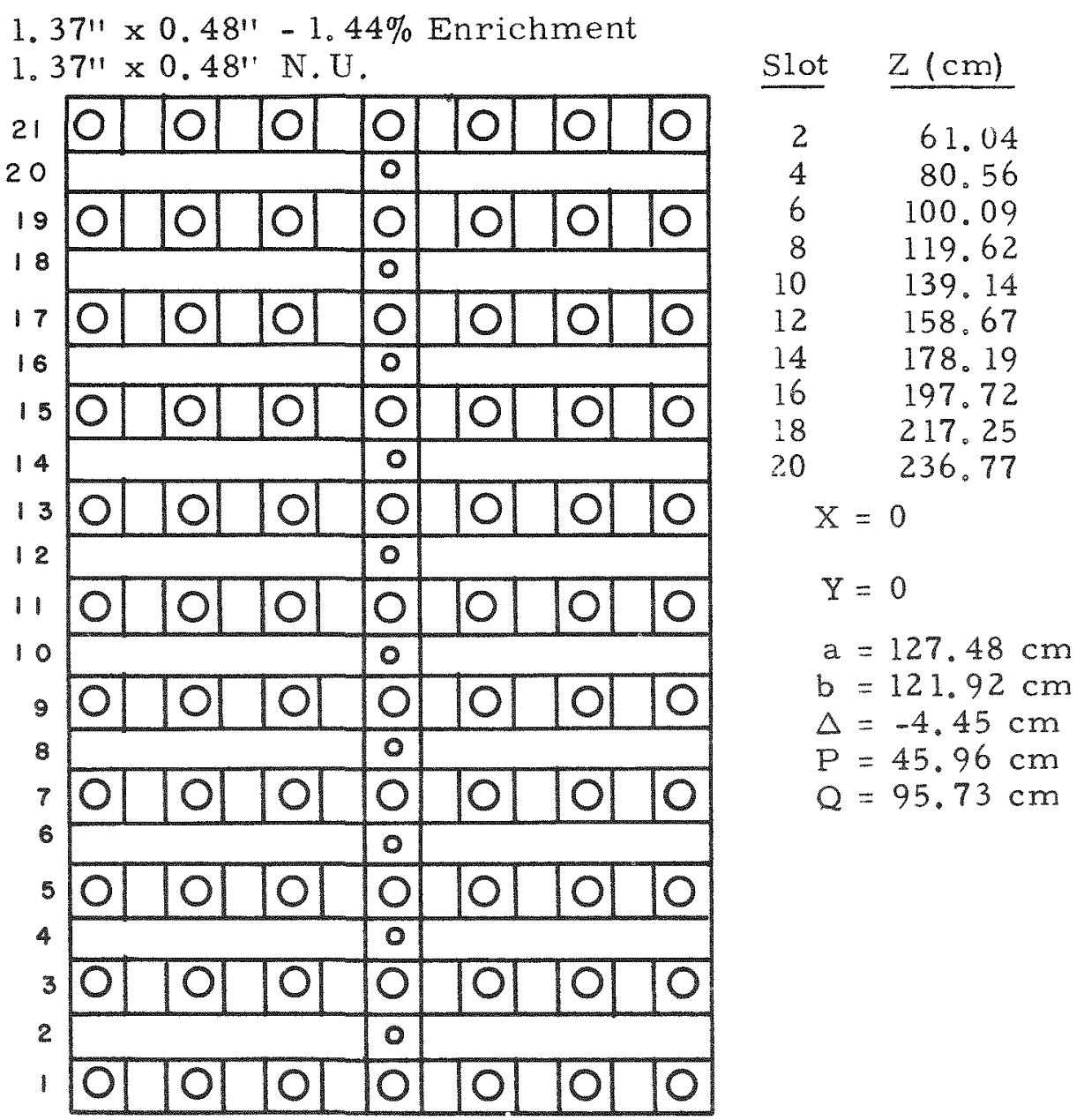

Row 1: $7-\left(4-3 / 16^{\prime \prime}\right)^{2}$ tube bars;

$6-\left(3-1 / 2^{\prime \prime} \times 4-3 / 16^{\prime \prime}\right)$

Row 2: $10-(3-1 / 2 " 1 \times 4-3 / 16 " 1)$;

$4-\left(1 " \times 3-1 / 2^{\prime \prime}\right) ; 1-(3-1 / 2 " 1 \times 4-3 / 16 " \prime)$ count bar。

FIGURE III-15

7-11/16-Inch Lattice, 4-Foot Pile 
Fuel Element:

1.36" N.U.

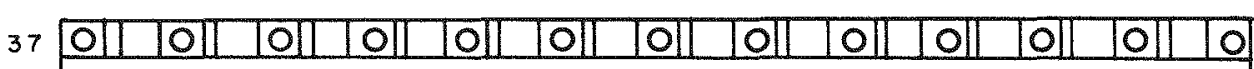

35

34

32

31

29

28

26

25

23

22

20

19

17

16

14

13

11

10

8

7

5

4

2

1

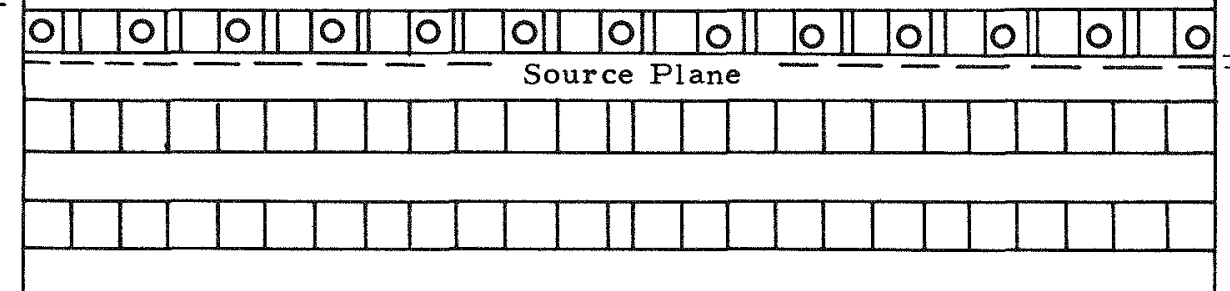

Slot $\quad Z(\mathrm{~cm})$

$11 \quad 76.84$

97.16

117.48

137.80

158. 12

178.44

198.76

$\mathrm{x}=0$

$Y=0$

$a=252.73 \mathrm{~cm}$

$\mathrm{b}=243.84 \mathrm{~cm}$

$\Delta=-5.72 \mathrm{~cm}$

$\mathrm{P}=2.54 \mathrm{~cm}$

$Q=50.80 \mathrm{~cm}$

Row 1: $13-(3-1 / 2 ")^{2}$ tube bars; $12-\left(3-1 / 2^{\prime \prime}\right)^{2}$;

$12-\left(1^{\prime \prime} \times 3-1 / 2^{\prime \prime}\right)$

Row 2: 3-1/2" fill layer.

Row 3: 1" fill layer.

FIGURE III-16

8-Inch Lattice, 8-Foot Pile 
Fuel Elements:

1. 36", N.U.

$1.66^{\prime \prime}$, N.U.

1. $37^{\prime \prime} \times 0.48^{\prime \prime}$, N.U.

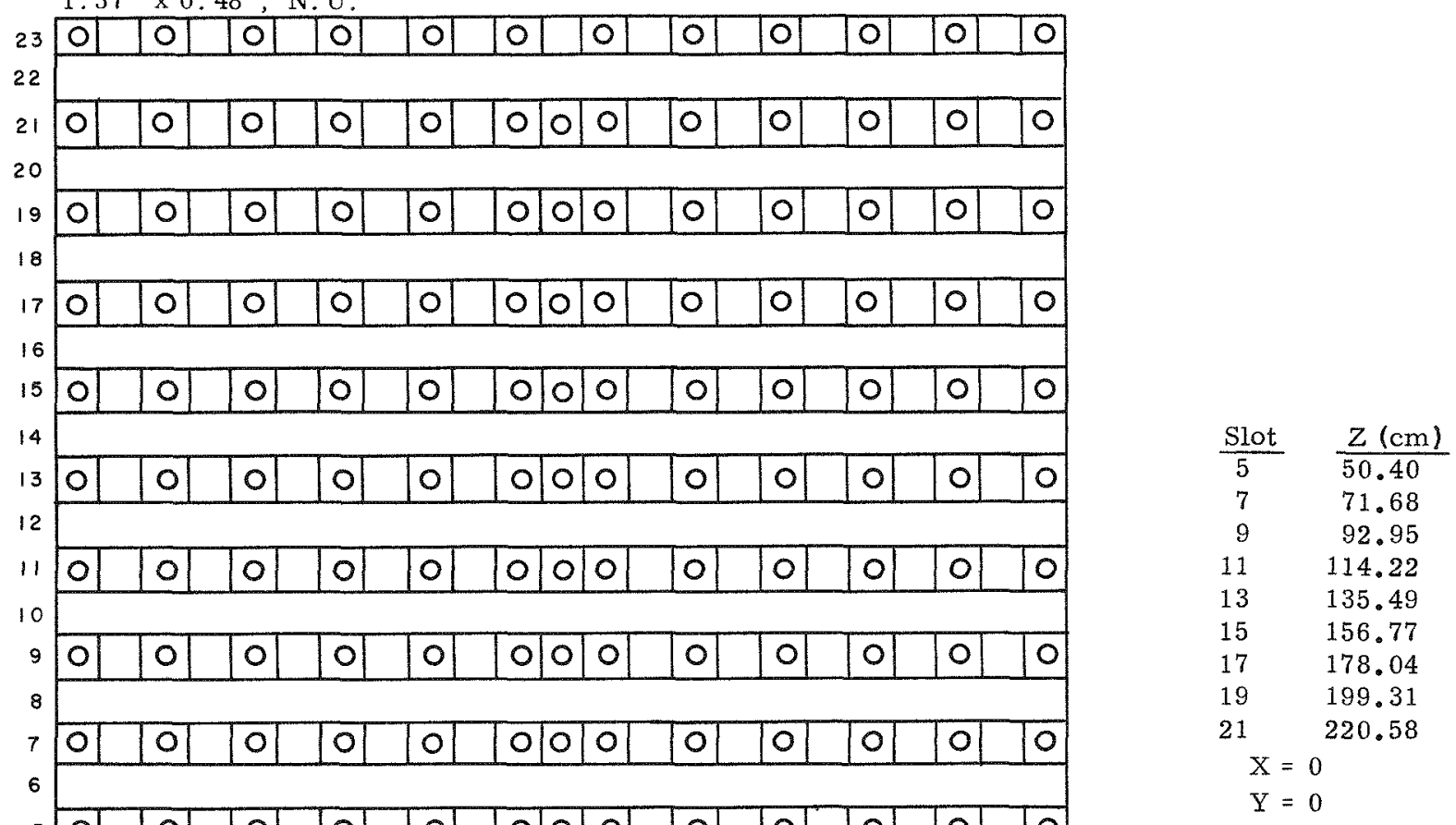

$a=244.63 \mathrm{~cm}$

$\mathrm{b}=243.84 \mathrm{~cm}$

$\Delta=-5.32 \mathrm{~cm}$

$\mathrm{P}=2.54 \mathrm{~cm}$

$Q=50.80 \mathrm{~cm}$
Row 1: $12-\left(4-3 / 16^{\prime \prime}\right)^{2}$ count bars; $11-\left(4-3 / 16^{\prime \prime}\right)^{2}$

Row 2: $\left(4-3 / 16^{\prime \prime}\right)^{2}$

FIGURE III-17

8-3/8-Inch Lattice, 8-Foot Pile 
Fuel Elements (All N。U。):

\begin{tabular}{|c|c|c|}
\hline Slot & $X(\mathrm{~cm})$ & $Z *(\mathrm{~cm})$ \\
\hline 1 & 0.0 & 51.27 \\
\hline 3 & $" 1$ & 72.55 \\
\hline 5 & $" 1$ & 93.89 \\
\hline 7 & $" 1$ & 115.09 \\
\hline 9 & $" 1$ & 136.36 \\
\hline 11 & $" 1$ & 157.64 \\
\hline 13 & $" 1$ & 178.91 \\
\hline 15 & $" 1$ & 200,18 \\
\hline 17 & $" 1$ & 221.45 \\
\hline 19 & $" 1$ & 242.73 \\
\hline $6-L 1$ & 5.32 & 104.45 \\
\hline L2 & 15.95 & "1 \\
\hline L 3 & 26.59 & $" 1$ \\
\hline L4 & 37.23 & $"$ \\
\hline L5 & 47.86 & $" 1$ \\
\hline L6 & 58.50 & 11 \\
\hline L7 & 69.14 & $"$ \\
\hline 8 & 0 & 125.73 \\
\hline 8-Ll & 10.64 & $1 "$ \\
\hline L2 & 21.27 & $" 1$ \\
\hline L3 & 31.91 & $"$ \\
\hline L4 & 42.54 & 11 \\
\hline L 5 & 53.18 & $"$ \\
\hline L 6 & 63.82 & $" 1$ \\
\hline L7 & \multicolumn{2}{|l|}{$\mathrm{Y}=0$} \\
\hline \multicolumn{3}{|c|}{$\begin{array}{l}\text { * For } 1.92^{\prime \prime} \times 2.50 " \text { solid } \\
\text { fuel, add } 2.18 \mathrm{~cm} \text { to } \\
\text { each corresponding slot } \\
\text { to get the } \mathrm{Z} \text { values of the }\end{array}$} \\
\hline
\end{tabular}

$1.92^{\prime \prime}$

$2.50^{\prime \prime}$

$2.5^{\prime \prime} \times 2.0^{\prime \prime}$ with $1.66^{\prime \prime} \times 1.12^{\prime \prime}$

$2.5^{\prime \prime} \times 1.6^{\prime \prime}$

2. $5^{\prime \prime} \times 1.6^{\prime \prime}$ with $1.17^{\prime \prime}$

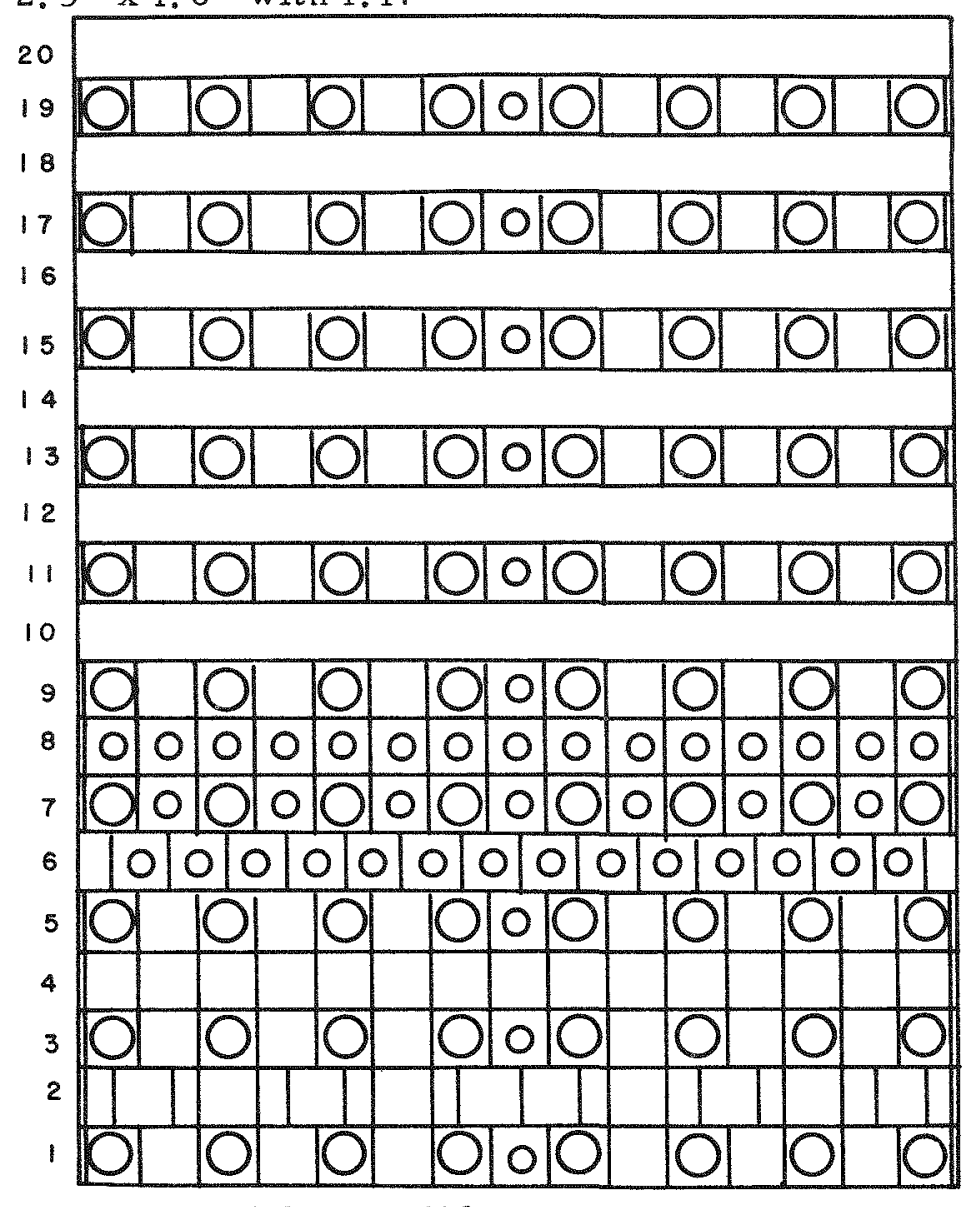

$\frac{1.92^{\prime \prime} \text { and } 2.5^{\prime \prime} \text { solid }}{\text { Row } 1: 8-\left(4-3 / 16^{\prime \prime}\right)^{2}}$ tube bars; $6-\left(4-3 / 16^{\prime \prime}\right)^{2}$; $1-\left(4-3 / 16^{\prime \prime}\right)^{2}$ count bar

Rows 2 and 6: $10-\left(4-3 / 16^{\prime \prime}\right)^{2} ; 10-\left(2^{\prime \prime} \times 4-3 / 16^{\prime \prime}\right)$;

1.92" 2.50" 2.5" hollow

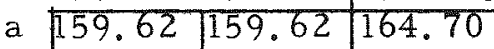

\begin{tabular}{ll|l|l} 
b & 121.92 & 121.92 & 121.92
\end{tabular}

$\begin{array}{llll}\Delta & -5.32 & -5.32 & -2.78\end{array}$

\begin{tabular}{l|l|l|l}
$\mathrm{P}$ & 45.96 & 45.96 & 48.14
\end{tabular}

\begin{tabular}{l|l|l|l}
$\mathrm{Q}$ & 95.73 & 95.73 & 95.73
\end{tabular}

$$
2-\left(1 / 2^{\prime \prime} \times 4-3 / 16^{\prime \prime}\right)
$$

2. 5" Hollow Fuel Elements

Same as 1.92" fuel, plus $2-\left(1 " \times 4-3 / 16^{\prime \prime}\right)$ solids in each layer

FIGURE III-18

8-3/8-Inch Lattice, 4-Foot Pile 


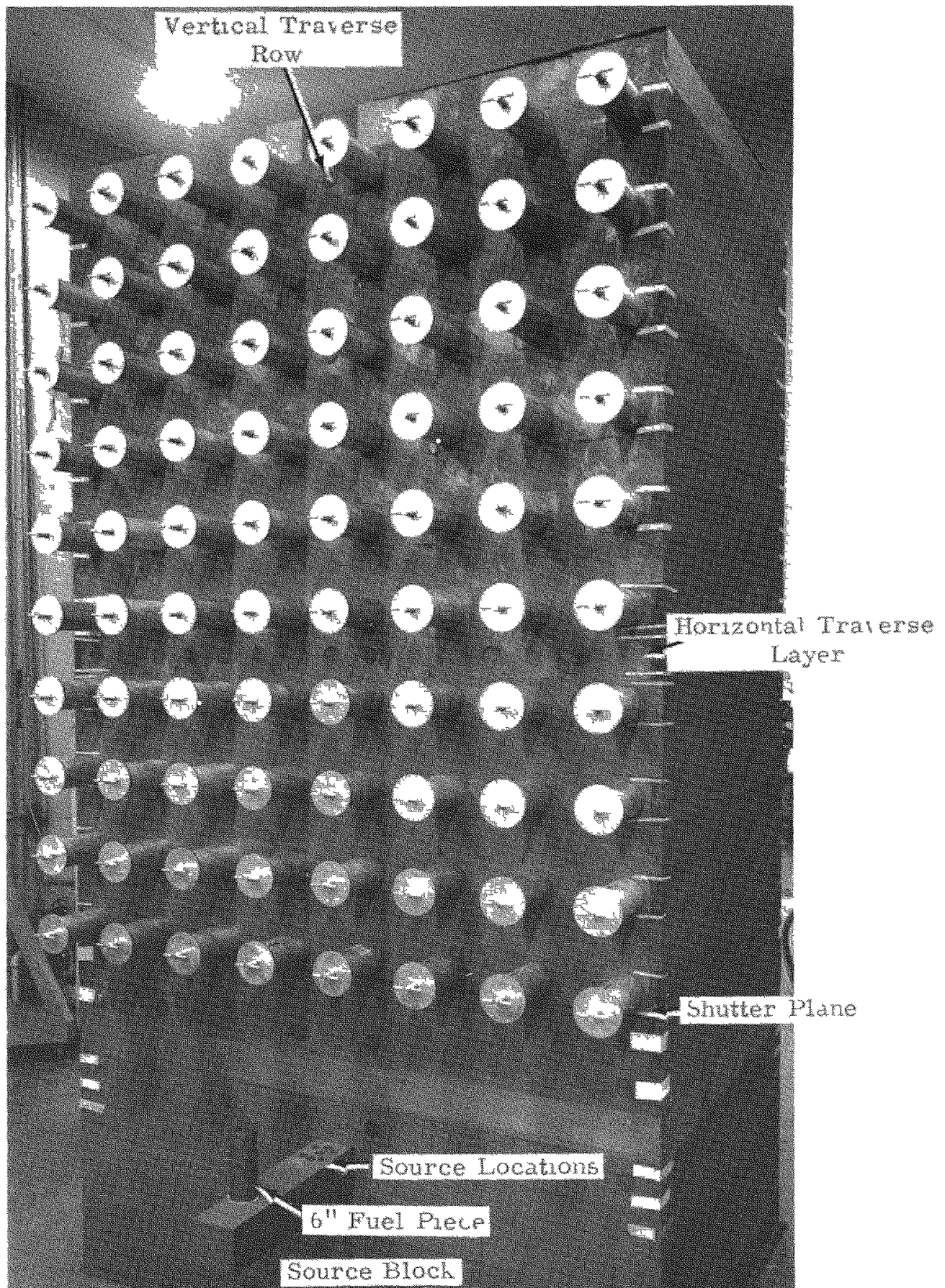

FIGURE III-18A

059089-2

8-3/8-Inch Lattice, 4-Foot Pile

2.5-Inch Solid Fuel 


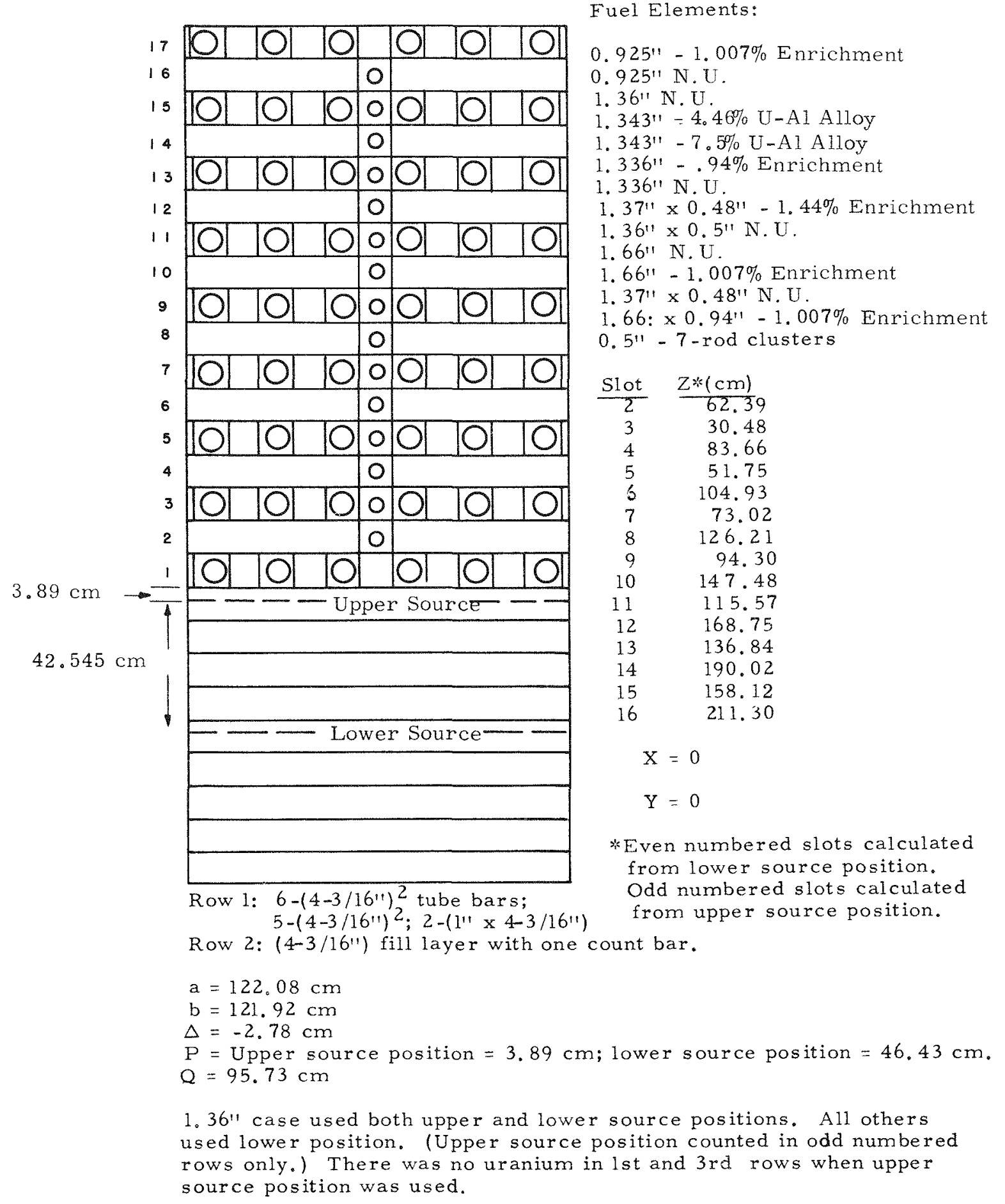

FIGURE III-19

8-3/8-Inch Lattice, 4-Foot Pile 
Fuel Element: $2.5^{\prime \prime} \times 2.0^{\prime \prime}$ with $1.66^{\prime \prime} \times 1.2^{\prime \prime}$ N. U.

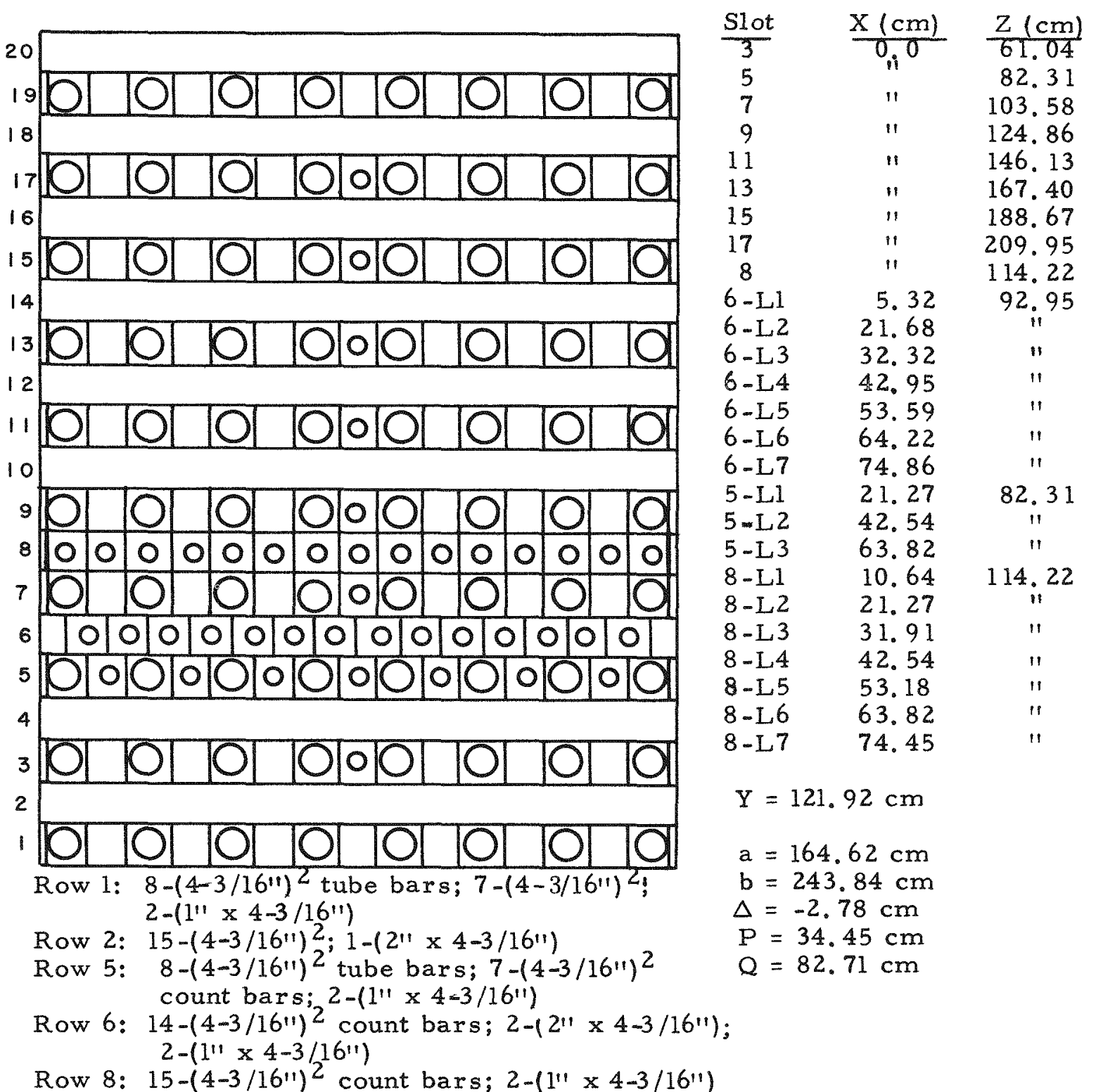

FIGURE III-20

8-3/8-Inch Lattice, 8-Foot Pile 


\begin{tabular}{|c|c|c|}
\hline Slot & $X(\mathrm{~cm})$ & $Z(\mathrm{~cm})$ \\
\hline 3 & 10.64 & 60.09 \\
\hline 5 & $n$ & 81.36 \\
\hline 7 & $n$ & 102.63 \\
\hline 9 & $" 1$ & 123.90 \\
\hline 11 & $"$ & 145.18 \\
\hline 13 & $\because$ & 166.45 \\
\hline 15 & "1" & 187.72 \\
\hline 17 & 11 & 208.99 \\
\hline $7-\mathrm{L1}$ & 10.64 & 102.63 \\
\hline $7-L_{2}$ & 31.91 & 11 \\
\hline $7-13$ & 53.18 & 11 \\
\hline $7-L 4$ & 74.45 & $"$ \\
\hline $7-L 5$ & 95.73 & $" 1$ \\
\hline $8-L 1$ & 7.86 & 113.27 \\
\hline $8-I 2$ & 18.49 & 11 \\
\hline $8-L 3$ & 29.13 & "1 \\
\hline $8-14$ & 39.77 & $" 1$ \\
\hline $8-L 5$ & 50.40 & 11 \\
\hline $8-1.6$ & 61.04 & 11 \\
\hline $8-\mathrm{L} 7$ & 71.68 & $"$ \\
\hline $8-L 8$ & 82.31 & $f 1$ \\
\hline $8-L 9$ & 92.95 & $n$ \\
\hline $8-L 10$ & 103. 58 & 11 \\
\hline 8-L11 & 114.22 & $" 1$ \\
\hline
\end{tabular}

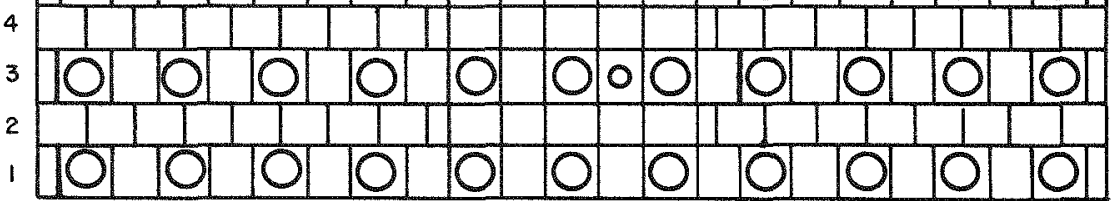

Row 1: $10-\left(4-3 / 16^{\prime \prime}\right)^{2} ; 11-\left(4-3 / 16^{\prime \prime}\right)^{2}$ tube bars; $2-\left(2^{\prime \prime} \times 4-3 / 16^{\prime \prime}\right)$ Row 2: $21-\left(4-3 / 16^{\prime \prime}\right)^{2} ; 2-\left(2^{\prime \prime} \times 4 \times 3 / 16^{\prime \prime}\right)$

Row 6: $21-\left(4-3 / 16^{\prime \prime}\right)^{2}$ count bars; $2-\left(2^{\prime \prime} \times 4-3 / 16 "\right)$

Row 7: $11-\left(4-3 / 16^{\prime \prime}\right)^{2}$ tube bars; $10-\left(4-3 / 16^{\prime \prime}\right)^{2}$ count bars; $2-\left(2^{\prime \prime} \times 4=3 / 16^{\prime \prime}\right)$

Row 8: $22-\left(4-3 / 16^{\prime \prime}\right)^{2}$ count bars.

Add $10.636 \mathrm{~cm}$ increments to obtain corresponding $X$ values in Row 6 . $\mathrm{Z}$ of Row 6 is $92.0 \mathrm{~cm}$.

Bottom row and two top rows are $2.5^{\prime \prime} \times 1.6^{\prime \prime}$ fuel (wet) used as buffers.

Both the buckling and cadmium ratio of the buffer layer are matched

to the tube-in-tube lattice.

$$
\begin{aligned}
& \mathrm{Y}=121.92 \mathrm{~cm} \\
& \mathrm{a}=233.52 \mathrm{~cm} \\
& \mathrm{~b}=243.84 \mathrm{~cm} \\
& \triangle=-0.24 \mathrm{~cm} \\
& \mathrm{P}=33.50 \mathrm{~cm} \\
& \mathrm{Q}=81.76 \mathrm{~cm}
\end{aligned}
$$

FIGURE III-21

8-3/8-Inch Lattice, 8-Foot Pile 
Fuel Elements:

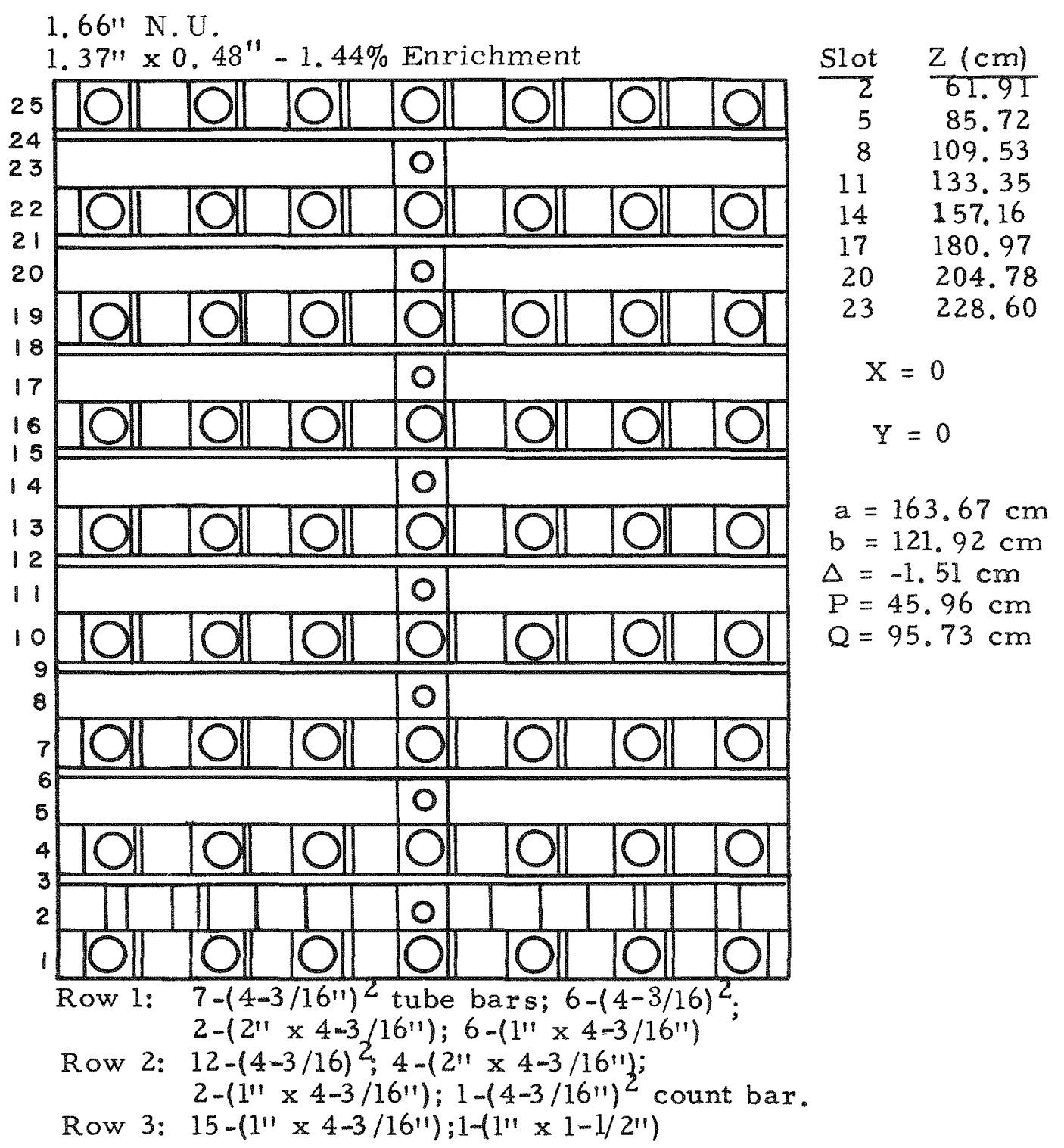

FIGURE III-22

9-3/8-Inch Lattice, 4-Foot Pile 


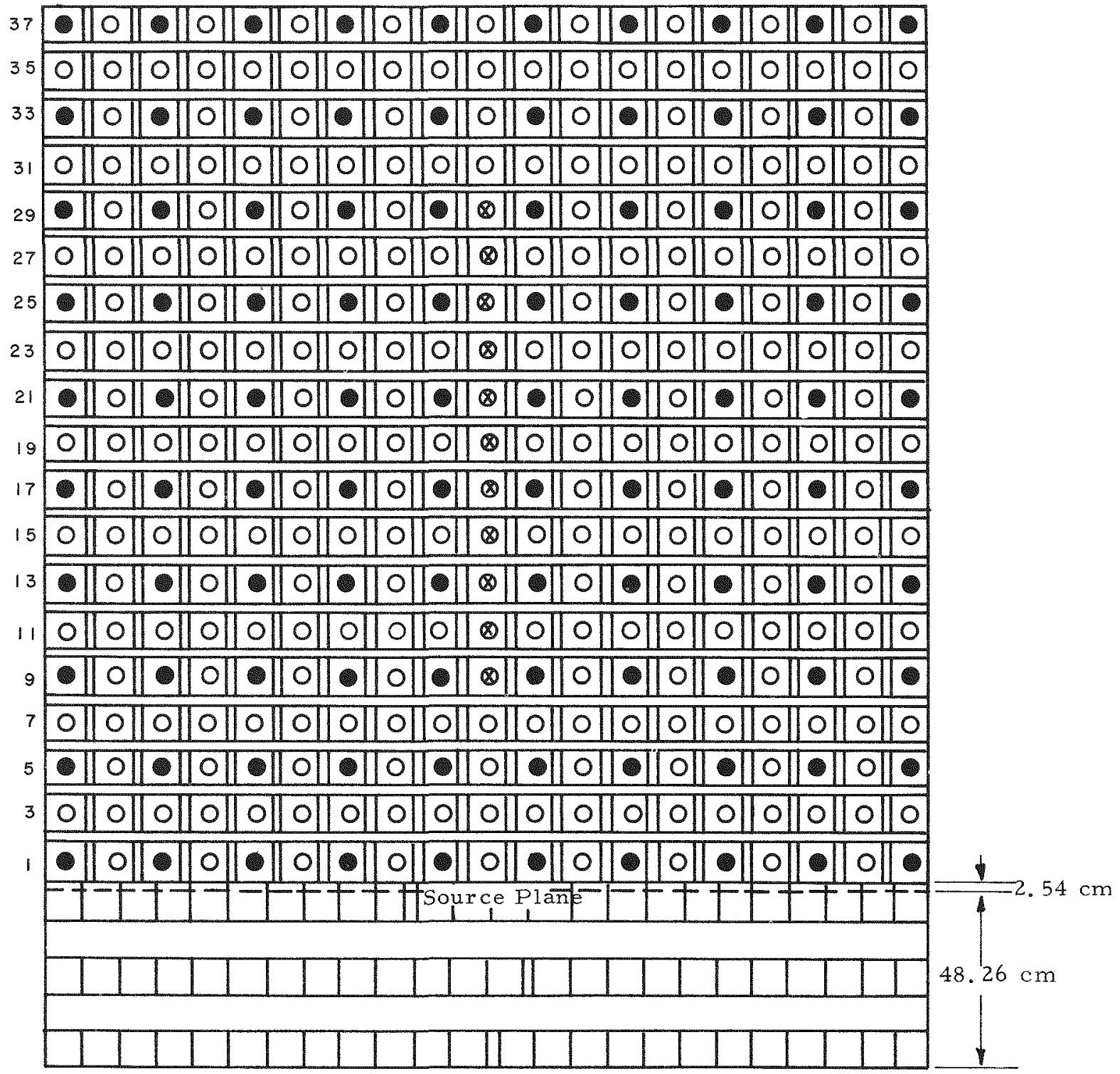

FIGURE III-23

10-3/8-Inch Lattice, 8-Foot Pile 


\section{TABLE 5}

\section{DATA FOR 10-3/8-INCH LATTICE, 8-FOOT PILE}

Fuel Elements:

$1.17^{\prime \prime}$, N.U.

$1.36^{\prime \prime \prime}$, N.U.

1. $66^{\prime \prime \prime}$, N.U.

$1.36^{\prime \prime \prime} \times 0.50^{\prime \prime}, \mathrm{N} . \mathrm{U}$.

$1.66^{\prime \prime} \times 0.81^{\prime \prime}$, N.U.

$1.66^{\prime \prime} \times 1.11^{\prime \prime}$, N.U.

$\begin{array}{lcr} & \begin{array}{c}\text { Slot } \\ \text { Sl }\end{array} & \frac{(\mathrm{cm})}{60.55} \\ & 11 & 73.72 \\ \text { N.U. } & 13 & 86.90 \\ \text { N.U. } & 15 & 100.07 \\ \text { N.U. } & 17 & 113.25 \\ & 19 & 126.43 \\ & 21 & 139.60 \\ & 23 & 152.78 \\ & 25 & 165.96 \\ & 27 & 179.13 \\ & 29 & 192.31 \\ & \text { X }=0 & \\ \text { Y }=0 & \end{array}$

$\mathrm{a}=247.81 \mathrm{~cm}$

$\mathrm{b}=243.84 \mathrm{~cm}$

$\Delta=-7.86 \mathrm{~cm}$

$\mathrm{P}=2.54 \mathrm{~cm}$

$\mathrm{Q}=50.80 \mathrm{~cm}$

Row 1: $19-\left(4-3 / 16^{\prime \prime}\right)^{2}$ tube bars;

$$
18-\left(1^{\prime \prime} \times 4-3 / 16^{\prime \prime}\right)
$$

Row 2: $1^{\prime \prime}$ fill layer

- Usual fuel arrangement

$\diamond$ Count bar positions

\section{FUEL ELEMENT ARRANGEMENTS}

$1.17^{\prime \prime}:$

1. Complete loading

1. $36^{\prime \prime}$ :

1. 60 tubes $1.36^{\prime \prime}$ rods in core buffered by 40 tubes of $1.36^{\prime \prime} \times 0.50^{\prime \prime}$ rods.

2. $1.36^{i \prime}$ rods in a complete loading.

1. $66^{\prime \prime}$ :

1. 42 tubes $1.66^{\prime \prime}$ buffered by 58 tubes of $1.66^{\prime \prime} \times 1.12^{\prime \prime}$ with $0.925^{\prime \prime}$ rods in the center

$1.36^{\prime \prime} \times 0.50^{\prime \prime}$ :

1. $1.36^{\prime \prime} \times 0.50^{\prime \prime}$ rods buffered by $1.66^{\prime \prime} \times 1.12^{\prime \prime}$ with $0.925^{\prime \prime}$ rods.

2. $1.36^{\prime \prime} \times 0.50^{\prime \prime}$ in core of pile buffered by $1.36^{\prime \prime}$ solid rods.

3. $1.36^{\prime \prime} \times 0.50^{\prime \prime}$ rods in sandwich loading similar to $5-3 / 16^{\prime \prime}$ lattice. $1.66^{\prime \prime} \times 0.81^{\prime \prime}$ :

1. 41 tubes of $1.66^{\prime \prime} \times 0.81^{\prime \prime}$ rods (dry) buffered by 59 tubes of $1.66^{\prime \prime}$ $\times 1.12$." with $0.925^{\prime \prime}$ rods.

2. 41 tubes $1.66^{\prime \prime} \times 0.81^{\prime \prime}$ wet buffered by 55 tubes of $1.36^{\prime \prime}$ rods.

$1.66 \times 1.12^{\prime \prime}$ : Complete loading 
Fuel Elements:

$1.36 " \mathrm{~N} . \mathrm{U}$.

$1.336 " 1-0.94 \%$ Enrichment

1. $37^{\prime \prime} \times 0.48 " \prime-1.44 \%$ Enrichment

1. $343^{\prime \prime}-4.46 \% \mathrm{U}$ - A1 Alloy

1. $34311-7.5 \% \mathrm{U}-\mathrm{Al}$ Alloy

$1.66^{\prime \prime}-1.607 \%$ Enrichment

$1.66^{\prime \prime} \times 0.94^{\prime \prime}-1.007 \%$ Enrichment Slot Z (cm)

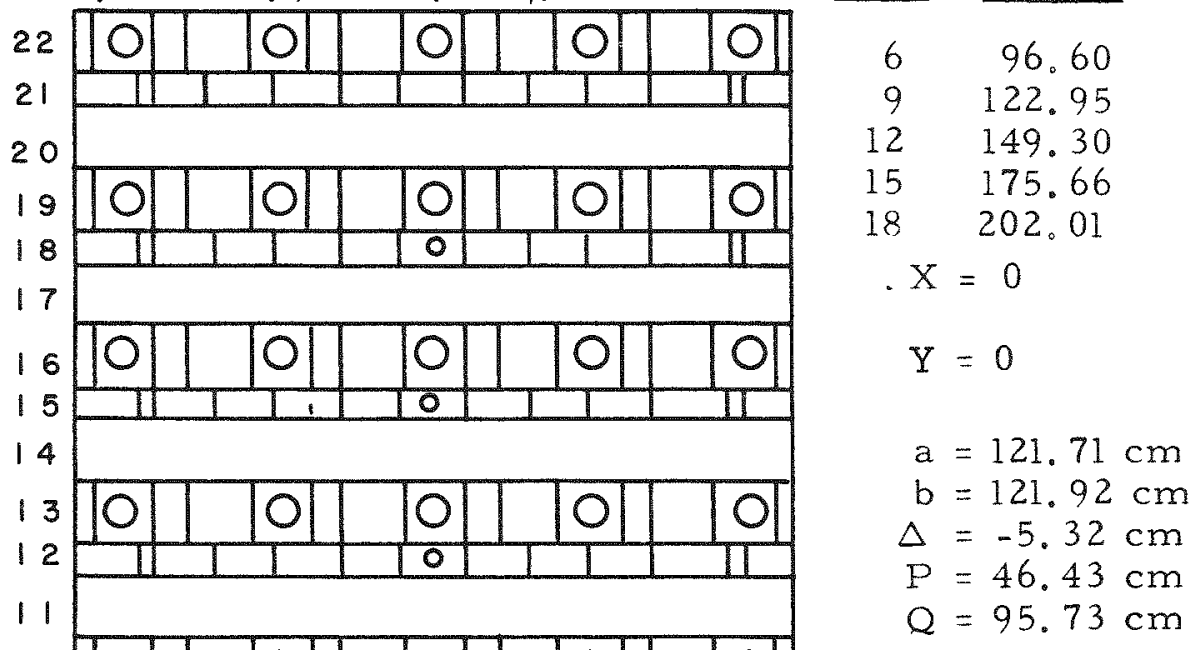

10

9

8

7

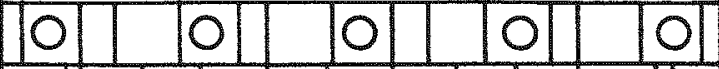

5

4

3

2

1

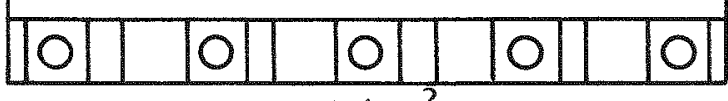

Row 1: $5-\left(4-3 / 16^{\prime \prime}\right)^{2}$ tube bars; $4-\left(2^{\prime \prime} \times 4-3 / 16^{\prime \prime}\right)$;

$$
2-\left(1 " 1 \times 4-3 / 16^{\prime \prime}\right)
$$

Row 2: $\left(4-3 / 16^{\prime \prime}\right)^{2}$ Fill Layer

Row 3: 11-(2"' x 4-3/16"'); 2-(1"' x 2"')

FIGURE III-24

10-3/8-Inch Lattice, 4-Foot Pile 
Fuel Element:

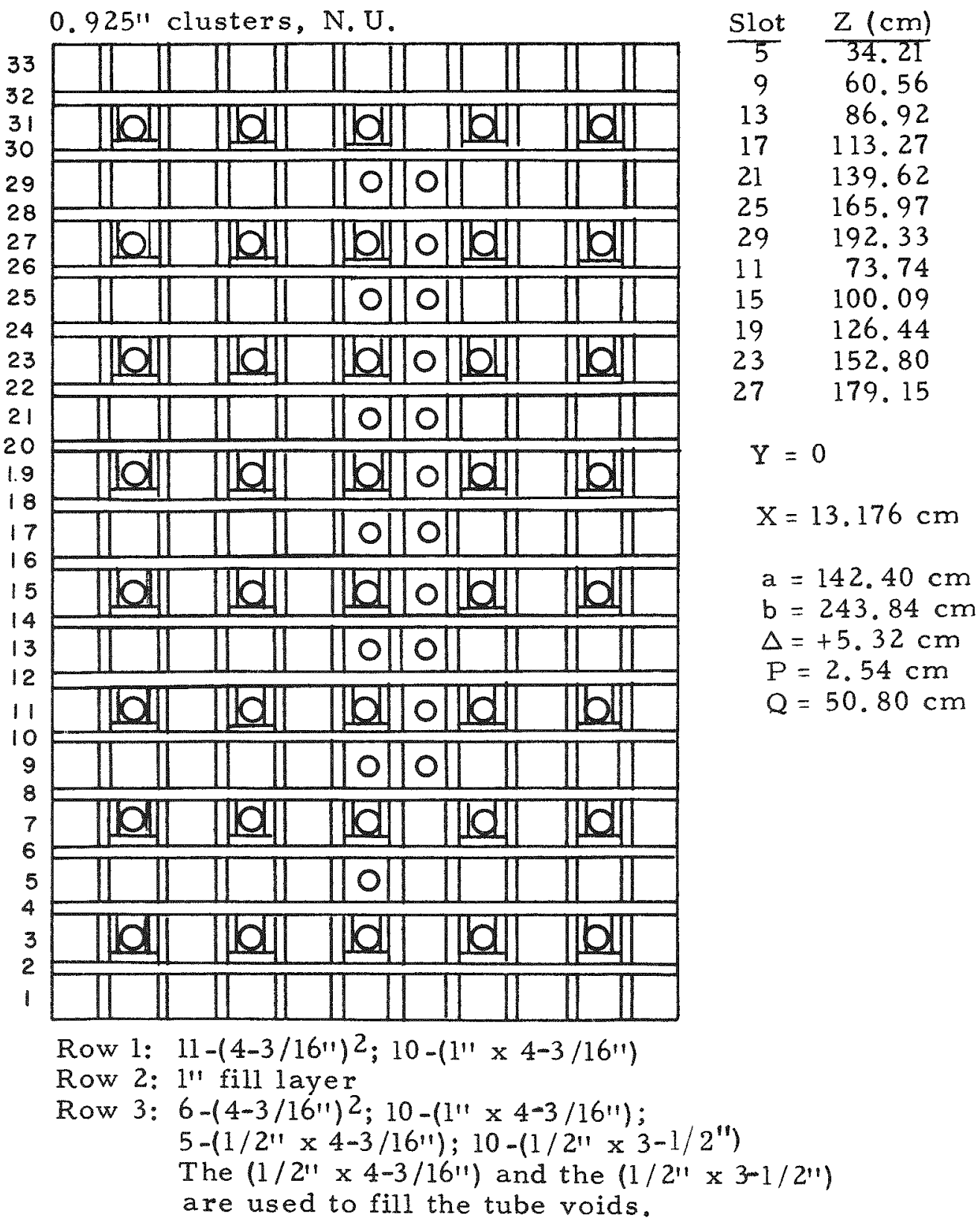

FIGURE III -25

10-3/8-Inch Lattice, 8-Foot Pile 


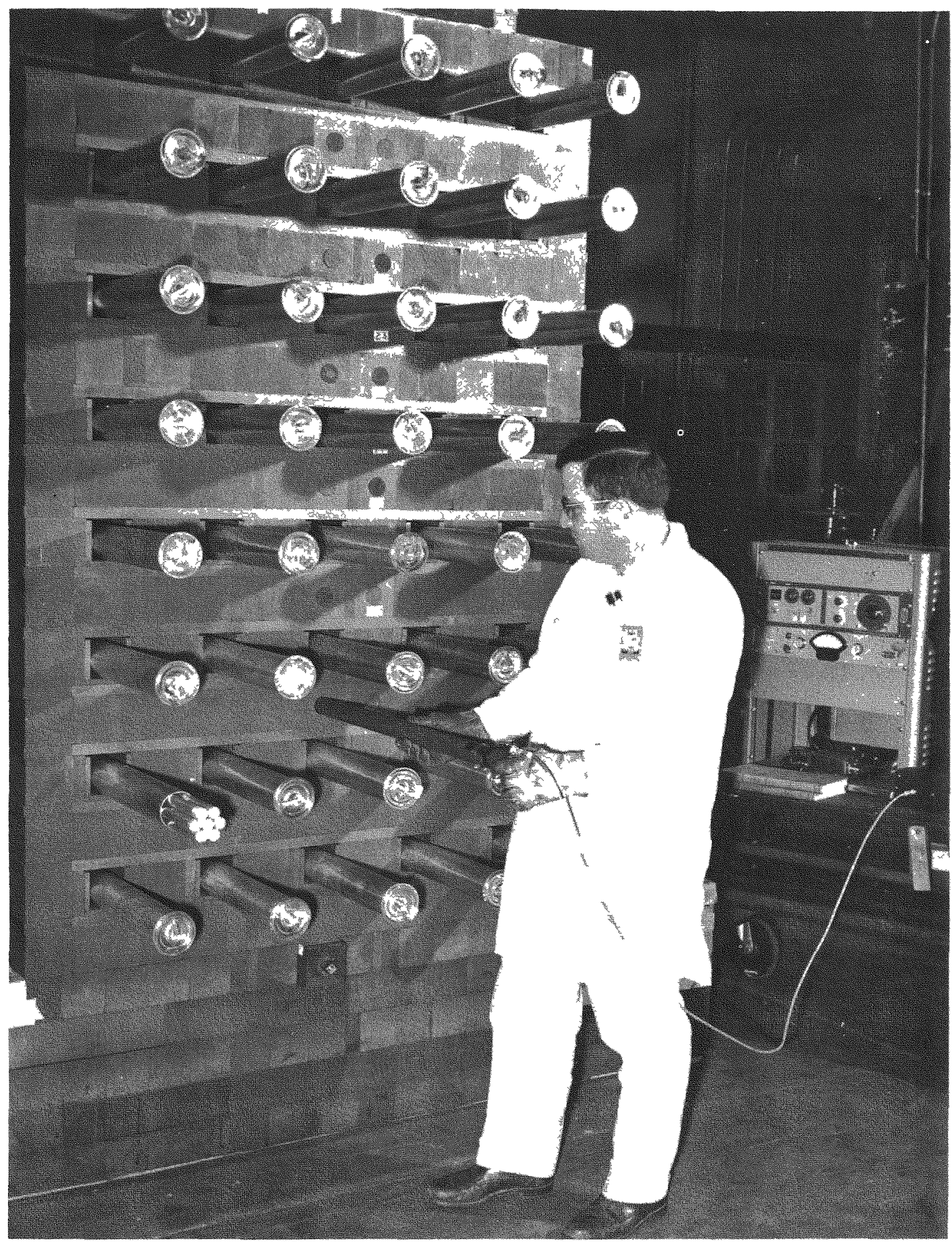

FIGURE III-25A

10-3/8-Inch Lattice, 8-Foot Pile 0.926 -Inch Rod Clusters

$0571465-2$ 
Fuel Elements (AI1 N. U.):

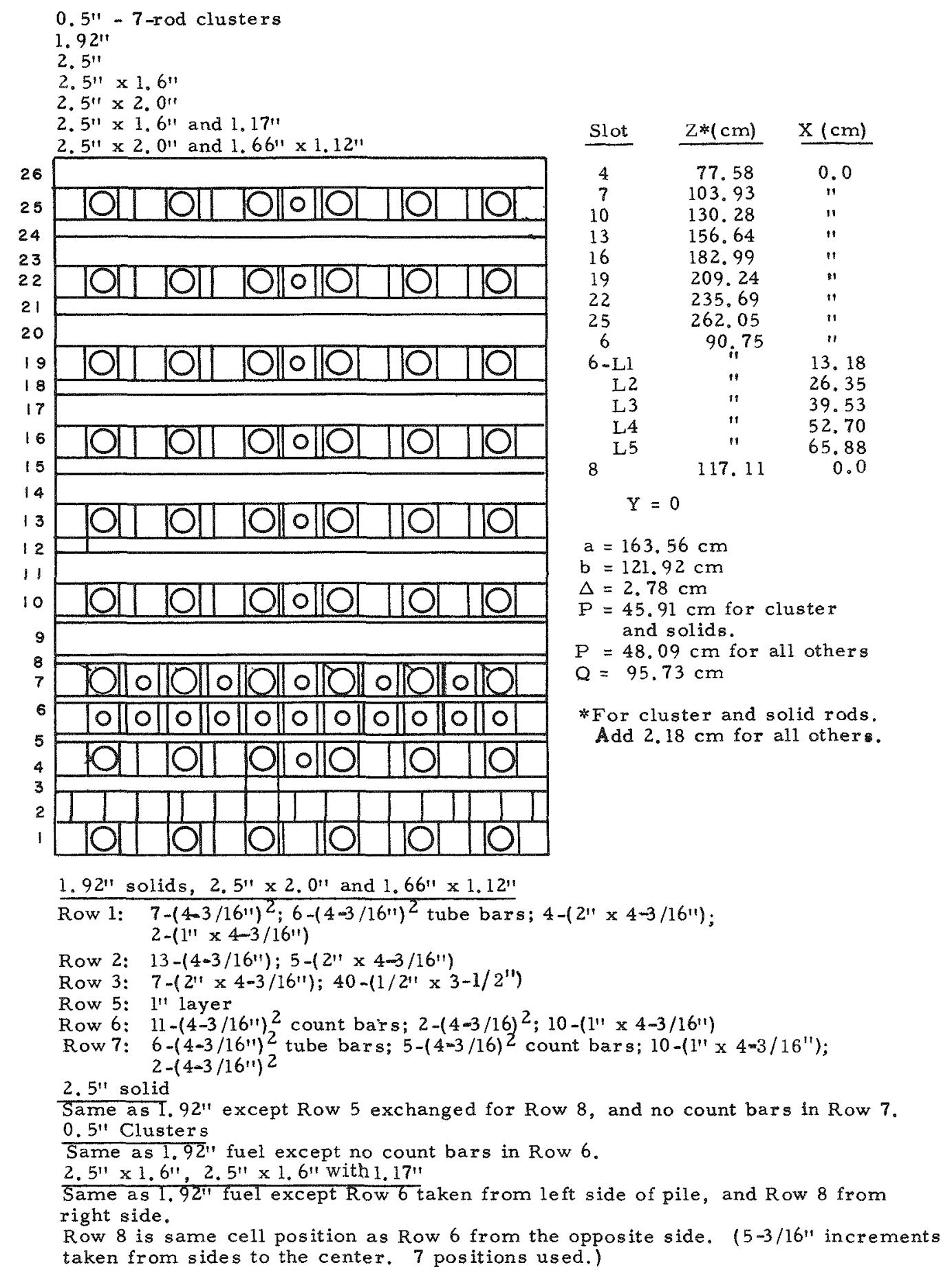

FIGURE III-26

10-3/8-Inch Lattice, 4-Foot Pile 
HW -69525

Fuel Elements:

1.921 N.U.

2. $50^{\prime \prime}$ N. U.

$2.5^{\prime \prime} \times 2.0^{\prime \prime}$ with $1.66^{\prime \prime} \times 1.12^{\prime \prime} \mathrm{N} . \mathrm{U}$.

$1.66^{\prime \prime}-1.007 \%$ Enrichment

2. $5^{11} \times 1.6^{\prime \prime} \mathrm{N}, \mathrm{U}$.

$2.5^{\prime \prime} \times 1.6 "$ with $1.17 " \mathrm{~N}$.U.

$1.66^{\prime \prime} \times 0.94^{\prime \prime}-1.007 \%$ Enrichment

23

22

21
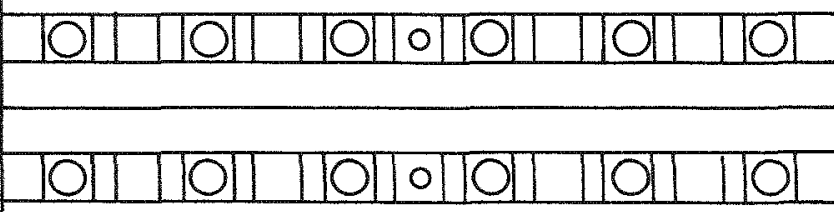

18

17

16

15

14

13

11

10

9

8

7

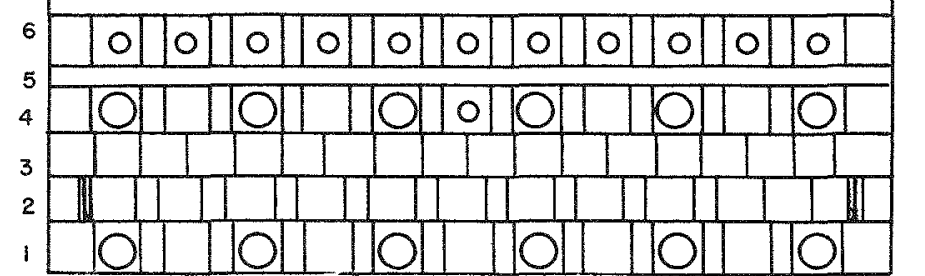

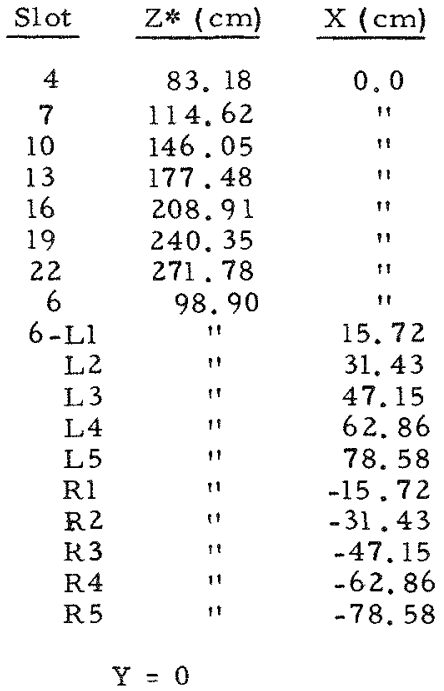

*For $1.92 "$ and 2. 5" solid. Add $2.18 \mathrm{~cm}$ for all others.

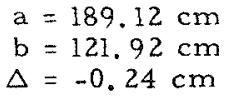

The $z$ values for the $2.50 " 1 \times 2.00 "$ $1.66^{\prime \prime} \times 1.12^{\prime \prime}$ fuel have an additional increment of $2.18 \mathrm{~cm}$ per slot.

Row 1: $7-\left(4-3 / 16^{\prime \prime}\right)^{2} ; 6-\left(4-3 / 16^{\prime \prime}\right)^{2}$ tube bars; $10-\left(2^{\prime \prime} \times 4-3 / 16^{\prime \prime}\right)$

Row 2: $12-\left(4-3 / 16^{\prime \prime}\right) 2 ; 11-(" 1 \times 4-3 / 16 \prime 1) ; 4 J_{1} / 2 " 1 \times 4-3 / 16$ '

Row 3: $17(4 " \times 4-3 / 161) ; 8\left(1 / 2^{\prime \prime} \times 3-1 / 21\right)$

Row 6: $2-\left(4-3 / 16^{\prime \prime}\right)^{2} ; 10-\left(2^{\prime \prime} \times 4-3 / 16^{\prime \prime}\right) ; 11-\left(4-3 / 16^{\prime \prime}\right)^{2}$ count bars

Row 7: $2-\left(4-3 / 16^{\prime \prime}\right) 2^{3} ; 6-\left(4-3 / 16^{\prime \prime}\right)^{2}$ tube bars; $5-\left(4-3 / 16^{\prime \prime}\right)^{2}$ count bars;

$10-\left(2 " 1 \times 4=3 / 16^{\prime \prime}\right)$

\section{FIGURE III-27}

12-3/8-Inch Lattice, 4-Foot Pile 
Fuel Element (A11 N. U.):

$1.66^{\prime \prime}$

$1.66^{\prime \prime} \times 0.81^{\prime \prime}$

$1.66^{\prime \prime} \times 1.12^{\prime \prime}$

1. $36^{\prime \prime}$

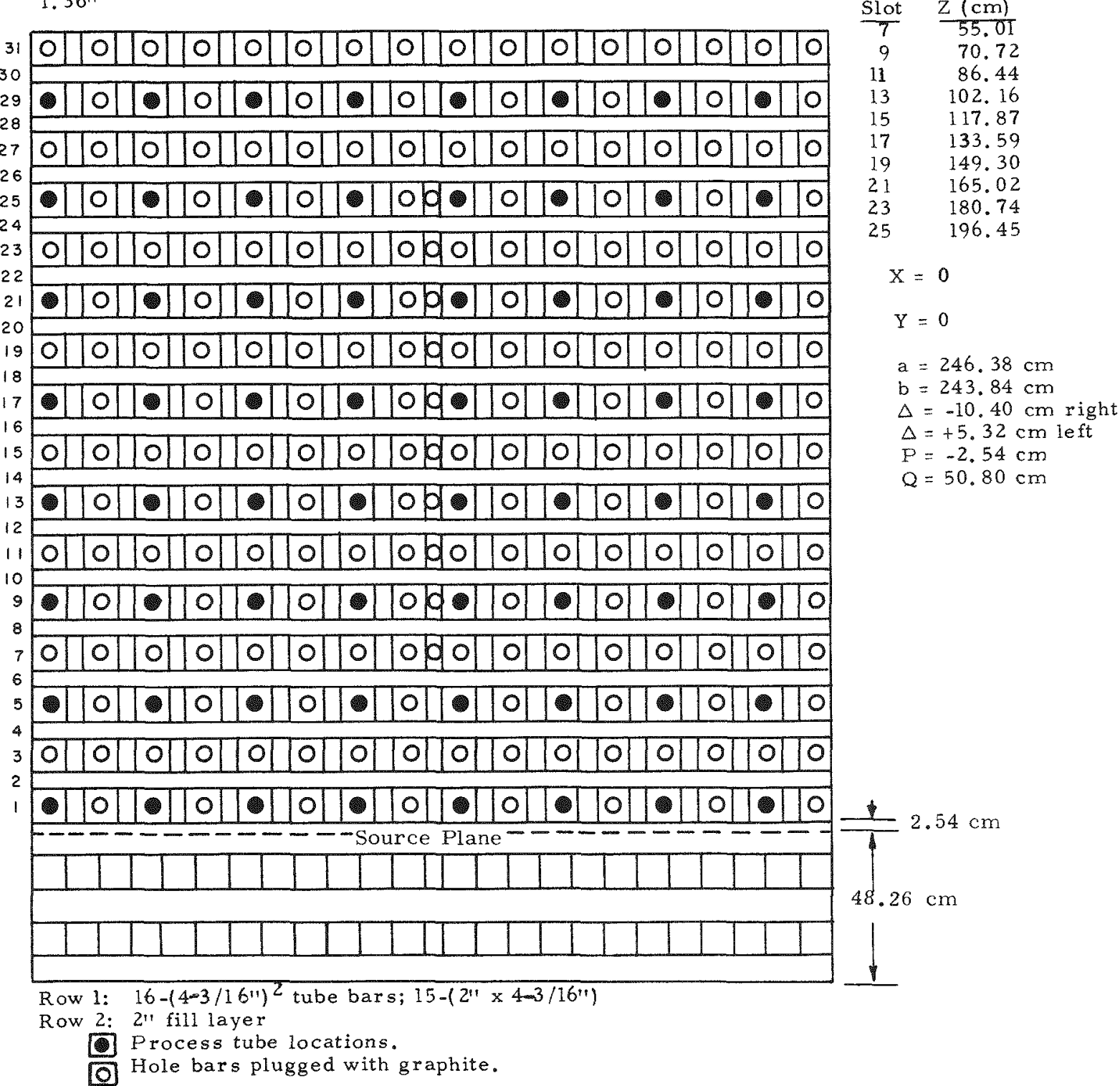

- 1 Process tube locations.

[0] Hole bars plugged with graphite.

FIGURE III-28

12-3/8-Inch Lattice, 8-Foot Pile 
Fuel Elements:

$0.925^{\prime \prime}$ clusters N.U.

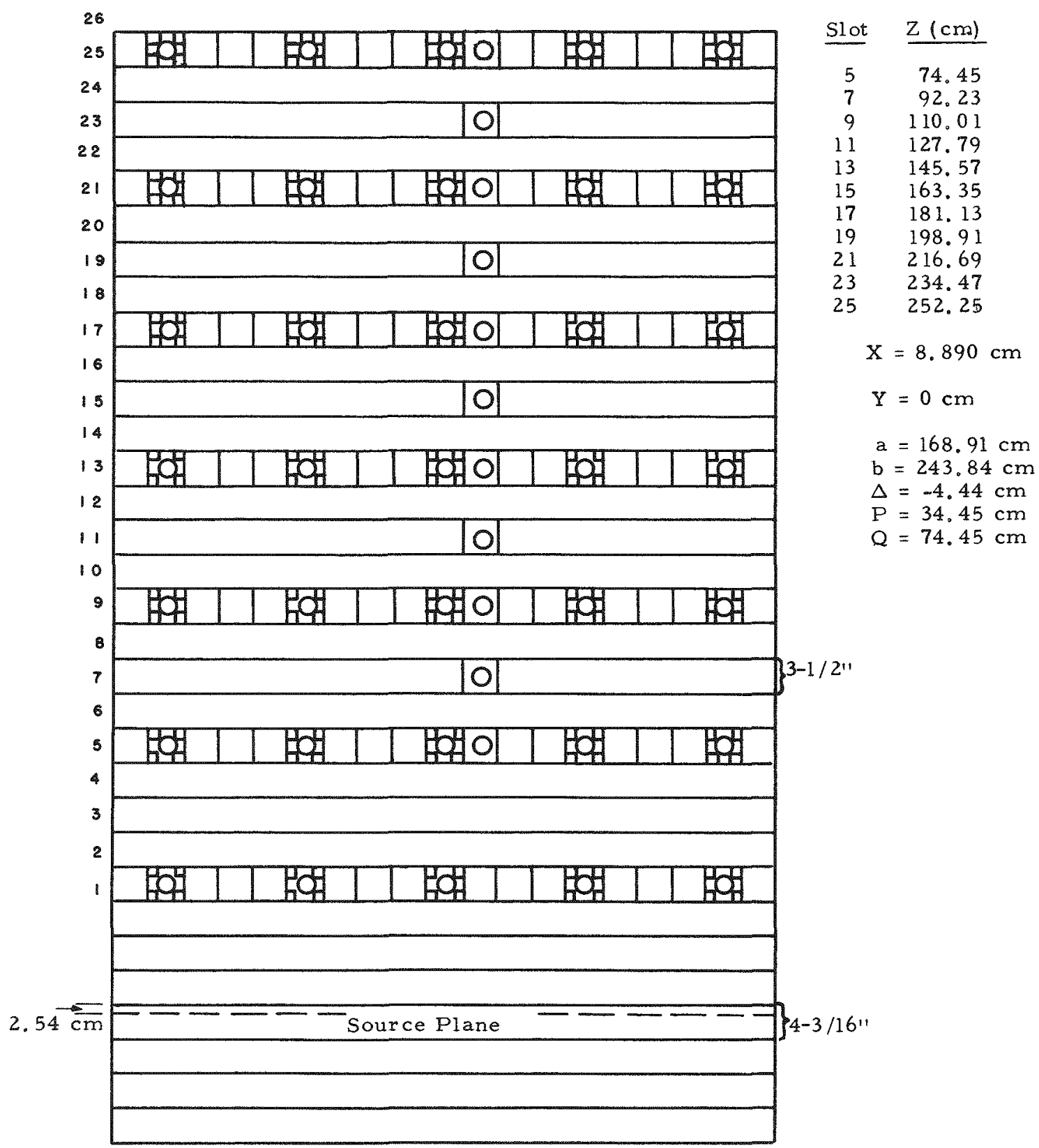

Row 1: $14-\left(3-1 / 2^{\prime \prime}\right) 2^{2} ; 5-\left(3-1 / 2^{\prime \prime}\right)^{2}$ tube voids with graphite in the corners. Row 2: $19-(3-1 / 2 " 1)^{2}$

FIGURE III - 29

14-Inch Lattice, 8-Foot Pile 
Fuel Elements (All N.U.):

2. $50^{\prime \prime}$

$2.5^{\prime \prime} \times 2.0^{\prime \prime}$ with $1.66^{\prime \prime} \times 1.12^{\prime \prime}$

$2.5^{\prime \prime} \times 1.6^{\prime \prime}$

2. 5" $\times 1.6^{\prime \prime}$ with 1.17 "

2. 5" $\times 1$. 6" with $0.5^{\prime \prime}$

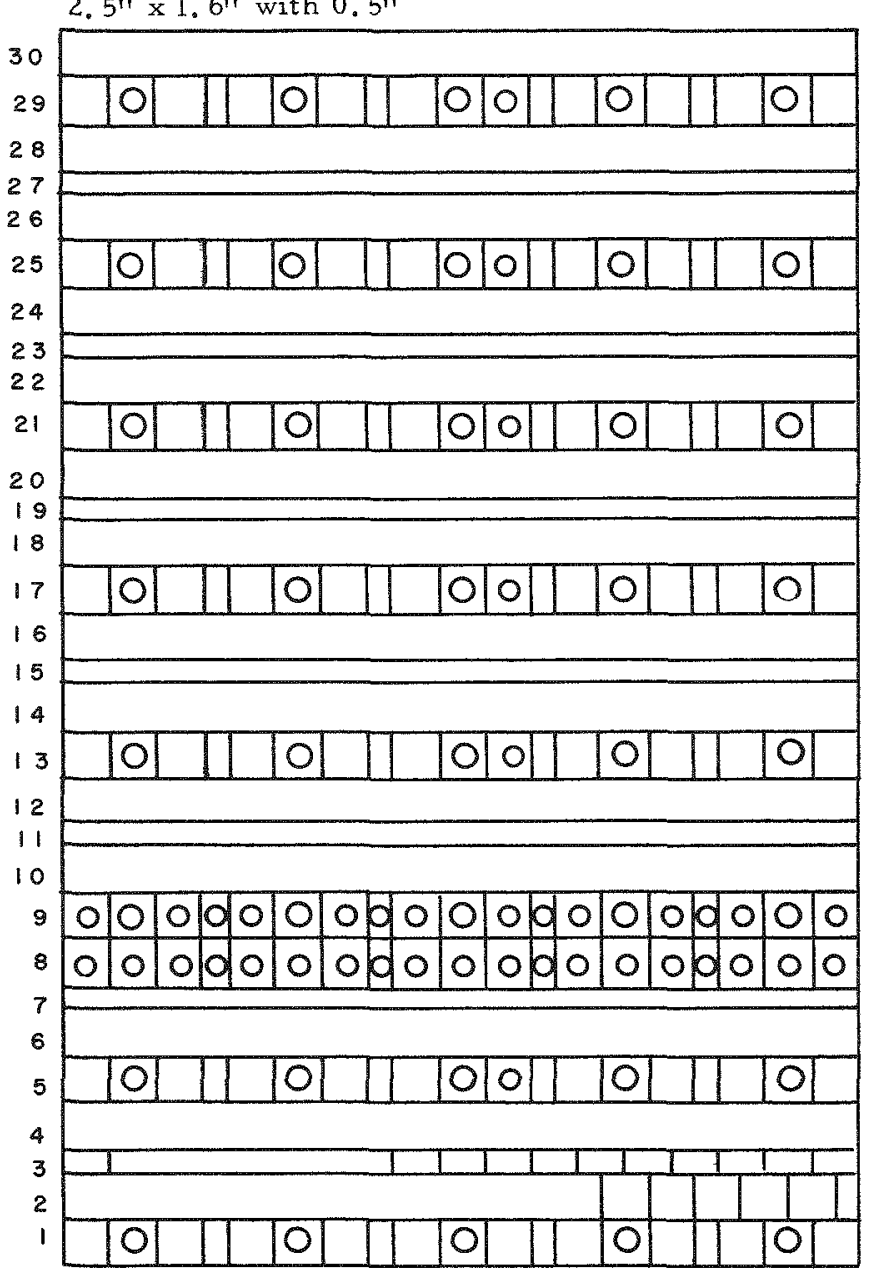

\begin{tabular}{|c|c|c|}
\hline Slot & $\mathrm{Z} *(\mathrm{~cm})$ & $X(\mathrm{~cm})$ \\
\hline 5 & 88.26 & 10.64 \\
\hline 9 & 125.25 & $"$ \\
\hline 13 & 162.24 & $"$ \\
\hline 17 & 199.23 & $"$ \\
\hline 21 & 236.22 & 11 \\
\hline 25 & 273.21 & $" 1$ \\
\hline 29 & 310.20 & $"$ \\
\hline 7 & $109.54 * *$ & 0.0 \\
\hline 8 & 114.62 & 0.0 \\
\hline $8-\mathrm{L1}$ & & 10.6 \\
\hline$L 2$ & " & 18.50 \\
\hline L3 & $"$ & 26.35 \\
\hline $\mathrm{L} 4$ & $"$ & 36.99 \\
\hline$L 5$ & $"$ & 47.62 \\
\hline L6 & $"$ & 55.48 \\
\hline 17 & $"$ & 63.34 \\
\hline L8 & $"$ & 73.98 \\
\hline L9 & $"$ & 84.61 \\
\hline $\mathrm{Rl}$ & $"$ & -10.64 \\
\hline $\mathrm{R} 2$ & $"$ & -18.49 \\
\hline R3 & $"$ & -26.35 \\
\hline $\mathrm{R} 4$ & $"$ & -36.99 \\
\hline R5 & $"$ & -47.62 \\
\hline R6 & " & -55.48 \\
\hline R7 & $"$ & -63.34 \\
\hline R8 & $"$ & -73.98 \\
\hline R9 & $"$ & -84.61 \\
\hline
\end{tabular}

* Add $2.18 \mathrm{~cm}$ for all except 2.50".

* F or Rows 7 and 8 interchanged.
$a=179.60 \mathrm{~cm}$
$\mathrm{b}=121.92 \mathrm{~cm}$
$\Delta=-2.54 \mathrm{~cm}$
$\mathrm{P}=48.14 \mathrm{~cm}$ for all
except 2.50"
$P=45.96 \mathrm{~cm}$ for $2.50^{\prime \prime}$
$Q=95.72 \mathrm{~cm}$

The $2.5^{\prime \prime} \times 1.6^{\prime \prime}, 2.5^{\prime \prime} \times 1.6^{\prime \prime}$ with $1.17^{\prime \prime}$, and the $2.5^{\prime \prime} \times 1.6^{\prime \prime}$ with $0.5^{\prime \prime}$ fuel elements have Rows 7 and 8 interchanged such that the counting positions are still in the $(4-3 / 16)^{2}$ count bars.

Row 1: $10-\left(4-3 / 16^{\prime \prime}\right)^{2} ; 5-\left(4-3 / 16^{\prime \prime}\right)^{2}$ tube bars; 4 (2' x 4-3/16")

Row 2: 4-(4-3/16"' $)^{2} ; 1\left(4^{\prime \prime} \times 4-3 / 16^{\prime \prime}\right) ; 1$ (2"' $\left.\times 4-3 / 16^{\prime \prime}\right)$ parallel to tubes: $\left.9+4^{\prime \prime} \times 4-3 / 16^{\prime}\right) ; 1-\left(4-3 / 16^{\prime \prime}\right)^{2} ; 2-\left(3-1 / 2^{\prime \prime} \times 4-3 / 16^{\prime}\right) ; 1$ (1/2" $\times$ $\left.4-3 / 16^{\prime \prime}\right)$ perpendicular to tubes.

Row 3: 11 (2"' $\left.\times 4-3 / 16^{\prime \prime}\right) ; 7$ (2"' $\left.\times 3=1 / 2^{\prime \prime \prime}\right)$

Row 8: $15-\left(4=3 / 16^{\prime \prime}\right)^{2}$ count bars; $4\left(2^{\prime \prime} \times 4-3 / 16^{\prime \prime}\right)$ count bars

Row 9: $10-\left(4-3 / 16^{\prime \prime}\right)^{2}$ count bars; $5-\left(4-3 / 16^{\prime \prime}\right)^{2}$ tube bars; $4\left\{2^{\prime \prime} \times 4-3 / 16^{\prime \prime}\right)$ count bars.

\section{FIGURE III-30}

14-9/16-Inch Lattice, 4-Foot Pile 


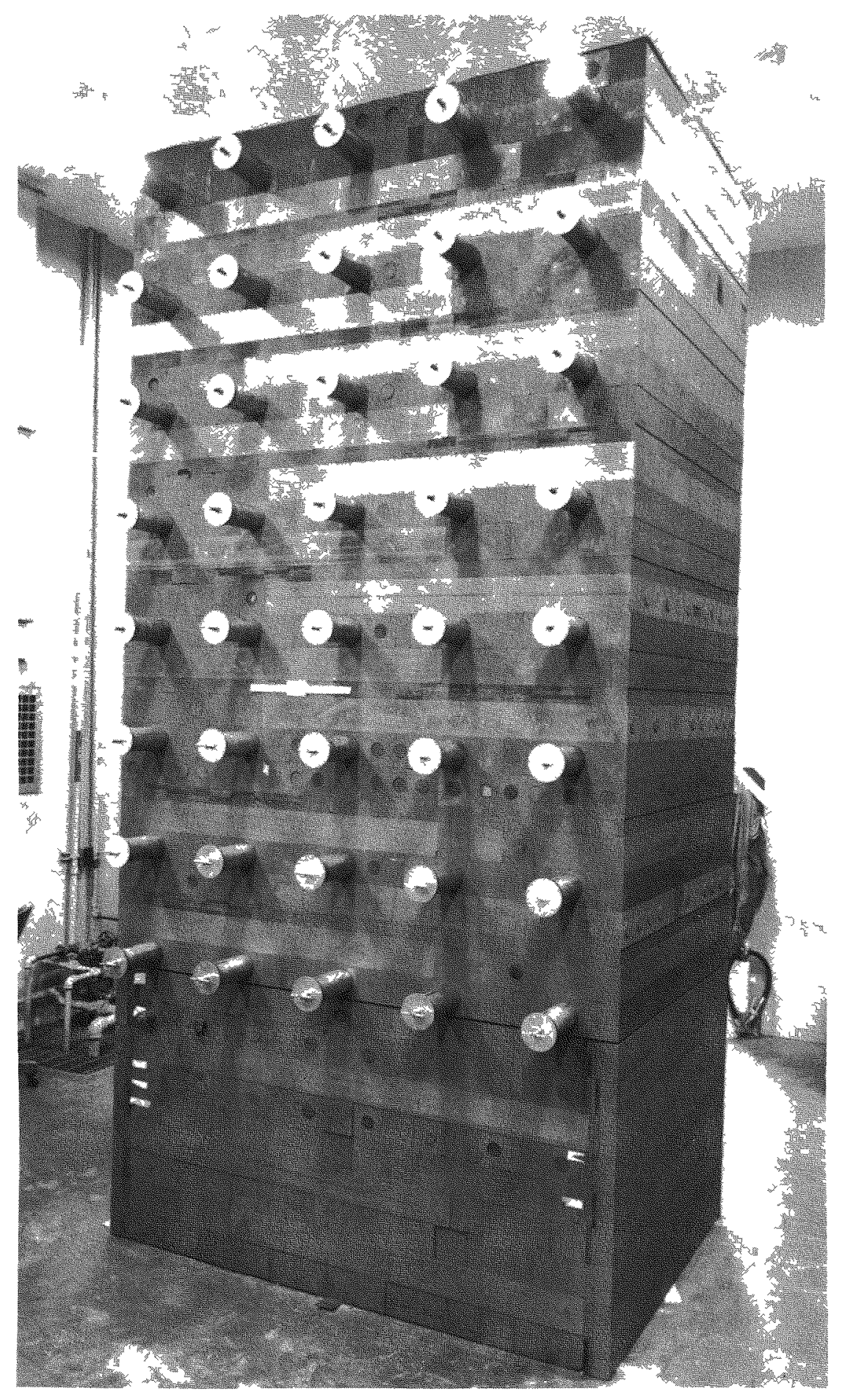

FIGURE III-30A 
Fuel Element: $2.5^{\prime \prime} \times 2.0^{\prime \prime}$ with $1.66^{\prime \prime} \times 1.12 " \mathrm{~N} . \mathrm{U}$.

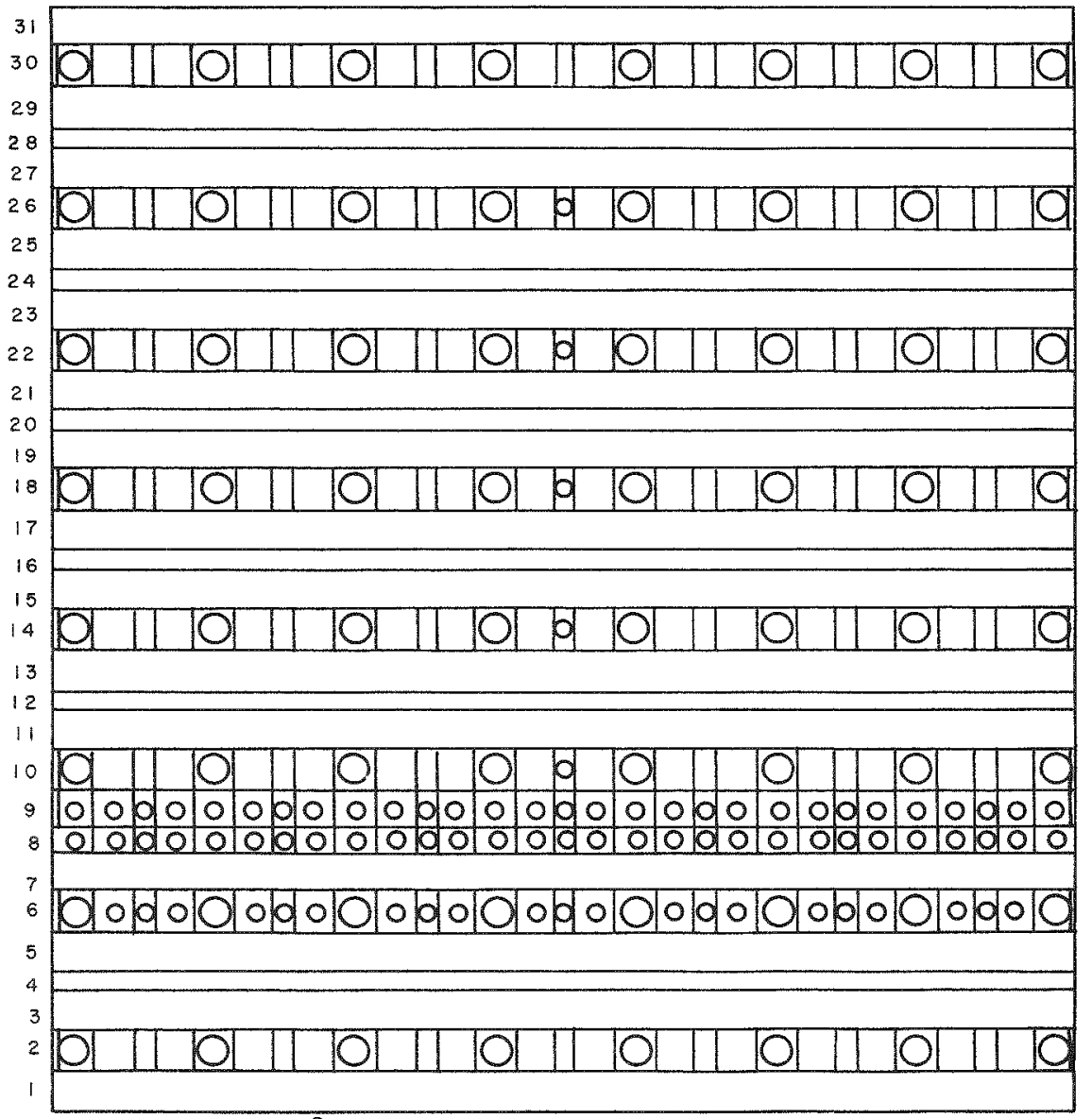

Row 1: $26-\left(3-1 / 2^{\prime \prime}\right)^{2}$

Row 2: $8-\left(4-3 / 16^{\prime \prime}\right)^{2}$ tube bars; $14-\left(4-3 / 16^{\prime \prime}\right)^{2} ; 7-\left(2^{\prime \prime} \times 4-3 / 16^{\prime \prime}\right)$ and $2-\left(1^{\prime \prime} \times 4-3 / 16^{\prime \prime}\right)$

Row 4: 2" fill layer

Row 6: $\quad 8-\left(4-3 / 16^{\prime \prime}\right)^{2}$ tube bars; $14-\left(4-3 / 16^{\prime \prime}\right)^{2}$ count bars; $7-\left(2^{\prime \prime} \times 4-3 / 16^{\prime \prime}\right)$ count bars and $2-\left(1 " \times 4-3 / 16^{\prime \prime}\right)$

Row 9: $22-\left(4=3 / 16^{\prime \prime}\right)^{2}$ count bars; $7-\left(2^{\prime \prime} \times 4 \times 3 / 16^{\prime \prime}\right)$ count bars and $2-\left(1 " \times 4-3 / 16^{\prime \prime}\right)$

Row 8: $22-\left(2^{\prime \prime} \times 4-3 / 16^{\prime \prime}\right)$ count bars; $7-\left(2^{\prime \prime}\right)^{2}$ count bars.

* $X$ values in Row 8 are same $X$ values as in Row 9

$Z(\mathrm{~cm})$ of Row 8 is $106.28 \mathrm{~cm}$.

This is the basic pile used in a study of the effect of adding graphite to the sides.

The measurements reported in Section II were made with 4" of graphite added

to each side of the pile, so that $\Delta=-0,476 \mathrm{~cm}$. 


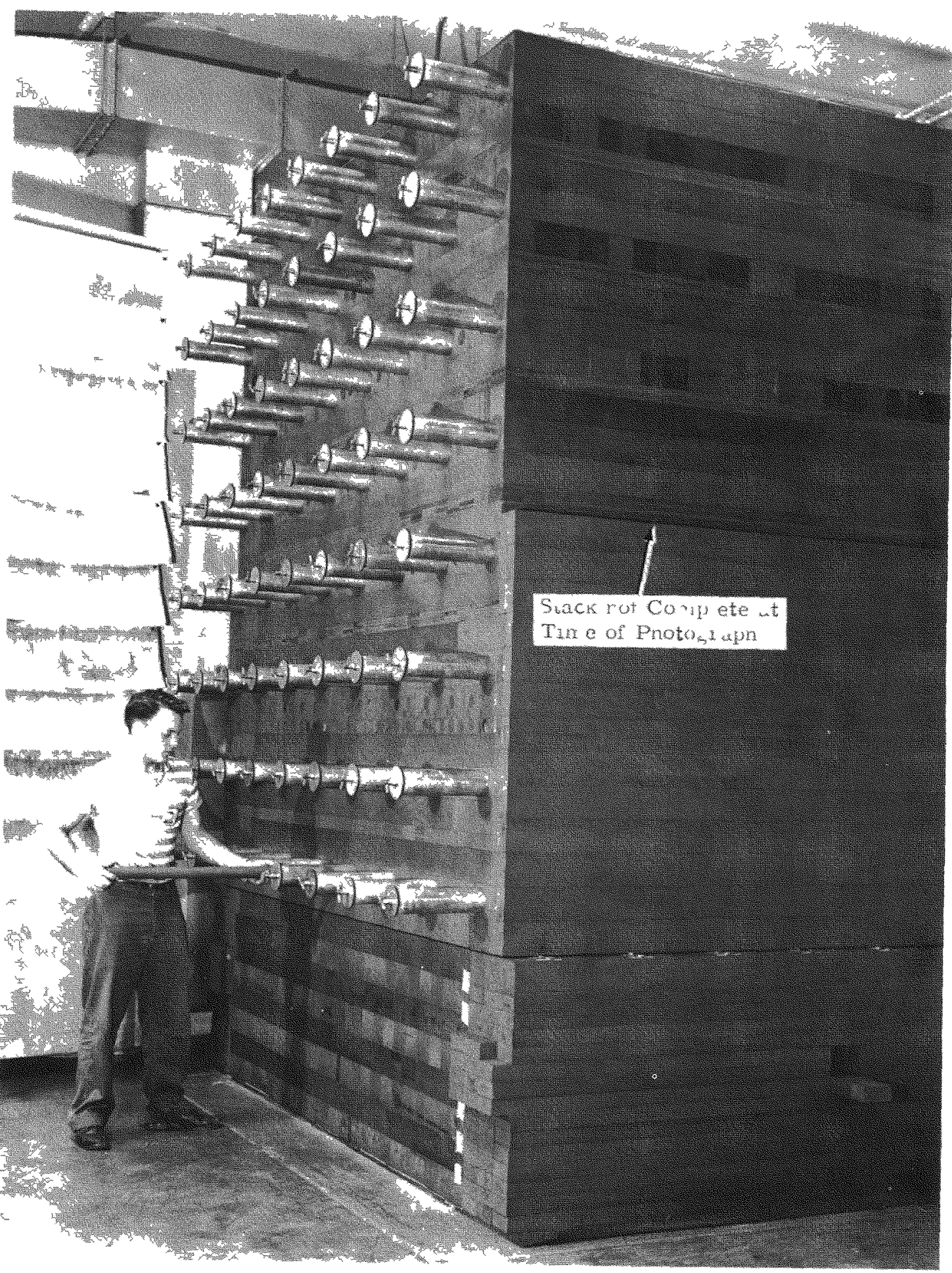

FIGURE III-31A

14-9/16-Inch Lattice, $10 \times 8$-Foot Pile

0601138-1 Tube-1n-Tube Fuel 
Fuel Elements:

$1.66^{\prime \prime}$ N. U.

$1.66^{\prime \prime} \times 0.81^{\prime \prime} \mathrm{N} . \mathrm{U}$.

$1.66^{\prime \prime} \times 1.12^{\prime \prime} \mathrm{N} . \mathrm{U}$.

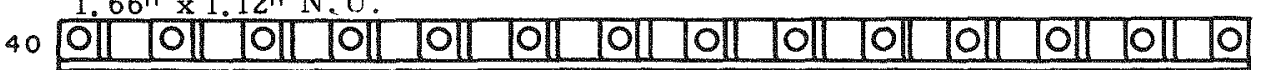

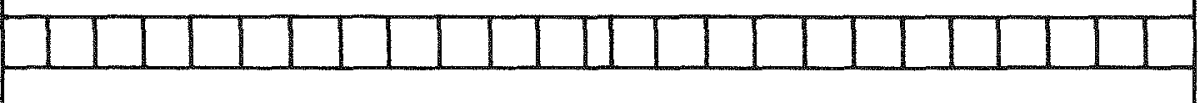

$a=256.54 \mathrm{~cm}$

$\mathrm{b}=243.84 \mathrm{~cm}$

$\Delta=$ left side $=4.44 \mathrm{~cm}$

$\Delta=$ right side $=-14.56 \mathrm{~cm}$

Row 1: $14-\left(3-1 / 2^{\prime \prime}\right)^{2}$ tube bars; $13-(3-1 / 21)^{2}$. $13-\left(1 / 2 " 1 \times 3-1 / 2^{\prime \prime}\right)$

$=2.54 \mathrm{~cm}$

$Q=50.80 \mathrm{~cm}$

Closed circles are fuel positions; open circles are hole bars plugged with graphite.

FIGURE III-32

15-Inch Lattice, 8-Foot Pile

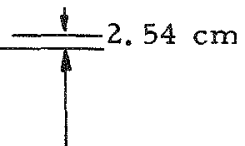

\section{$48.26 \mathrm{~cm}$}

Row 2: 3-1/2" fill layer.

Row 3: 1/2" fill layer.

Slot $Z(\mathrm{~cm})$

$14 \quad 92.08$

$17 \quad 111.12$

$20 \quad 130.18$

$23 \quad 149.22$

$26 \quad 168.28$

$29 \quad 187.32$

$32 \quad 206.38$

$X=-9.52 \mathrm{~cm}$

$Y=-6.35 \mathrm{~cm}$

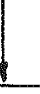


Fuel Element:

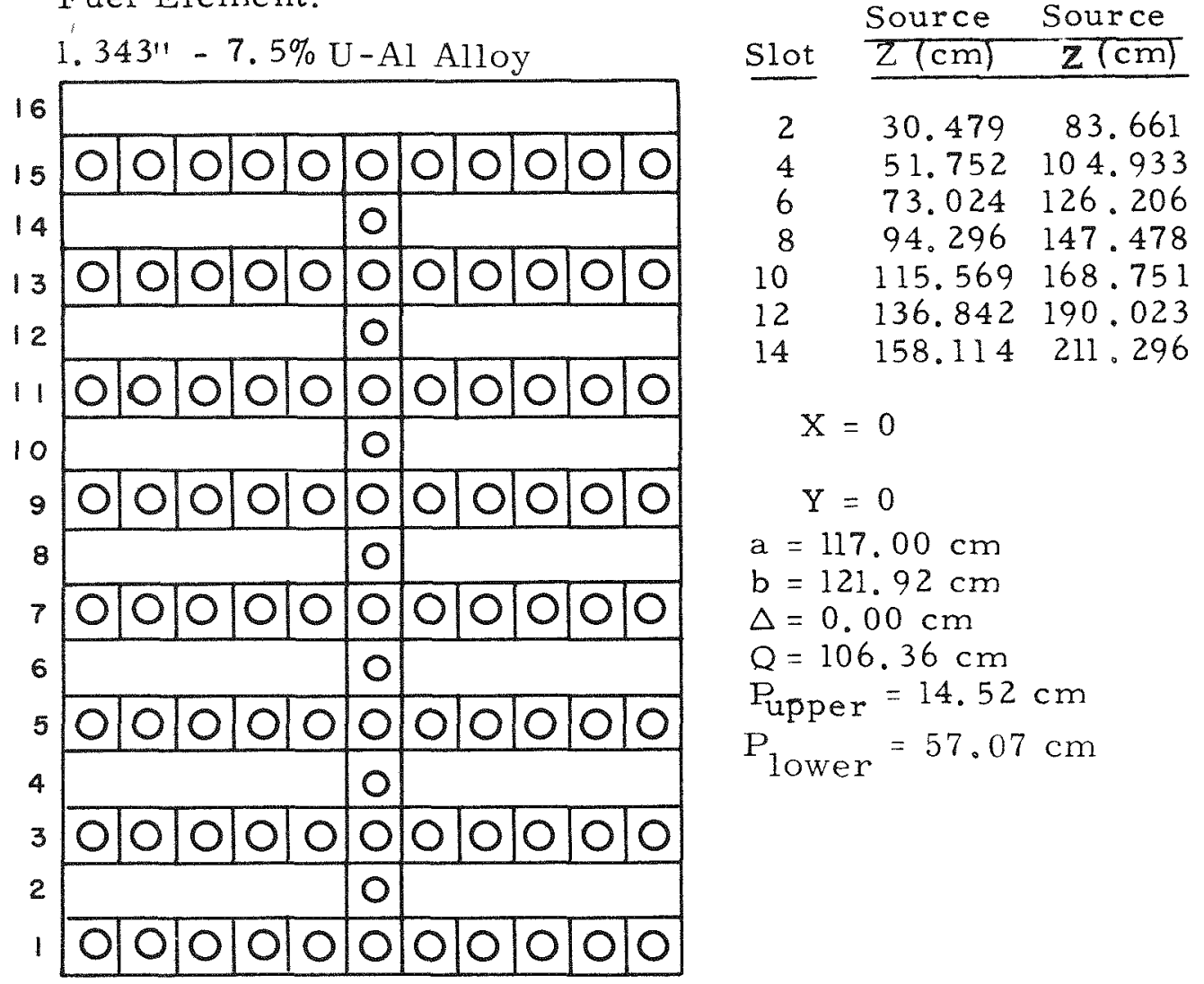

Row 1: $11-\left(4-3 / 16^{\prime \prime}\right)^{2}$ tube bars

Row 2: $10-(4-3 / 16)^{2} ; 1-(4-3 / 16)^{2}$ count bar

The tube rows alternate starting in the second row for the pile with sources in the lower position.

FIGURE III-33

4-3/16- x 8-3/8-Inch Lattice, 4-Foot Pile 


\section{ACKNOWLEDGEMENTS}

The author wishes to thank G. D. Seybold for his laborious compilation of most of the data included, which is actually the major part of this work. Another indispensable contributor was G. W. R. Endres who has worked with the exponentials since 1958 and guided the search through the original notebooks. It is impossible to acknowledge all of the workers who performed the measurements summarized here, but an indication of their contributions may be found in the references. 


\section{REFERENCES}

The first section of the references includes those Hanford reports and articles which contain exponential data on graphite-uranium systems.

The second section includes a chronological list of the "Nuclear Physics Research Quarterly Report" (formerly the "Quarterly Report of the Physics Unit" or "Physics Research Quarterly Report") and a guide to the monthly reports of the units responsible for exponential pile measurements.

1. Block, E. Z. Reconciliation of Sigma Pile and 105 Pile Graphite Diffusion Lengths by Application of Neutron Streaming Corrections, HW-29147. August 28, 1953. (CONFIDENTIAL).

2. Clayton, E. D. HW-28674. July 13, 53. (SECRET).

3. Clayton, E. D. Exponential Pile Measurements in Graphite-Uranium Lattices, AECD-3677. Also HW-31999, June 1, 1954.

4. Clayton, E. D. Exponential Pile Measurements with Hollow Slugs in Graphite-Uranium Lattices, HW-36174. Apri1 12, 1955.

5. Clayton, E. D. Exponential Measurements with Enriched Uranium in Graphite Lattices, HW-50257. May 17, 1957.

6. Clayton, E. D. "Buckling Measurements with Enriched Uranium in Graphite Systems, "in Proceedings of the French-American Conference on Graphite Reactors: p. 189, BNL-489. November 15, 1957.

7. Davenport, D. E. "Exponential Experiments in Graphite Systems," in Proceedings of the International Conference on the Peaceful Uses of Atomic Energy, Geneva, 1955, Vol. 5: 309.

8. Lee, M. T. Re-evaluation of Graphite Diffusion Lengths, HW-51175. June 5, 1957.

9. Lloyd, R. C. and C. R. Richey. Temperature Coefficient of a Graphite-Uranium Lattice, HW-51008. June 18, 1957.

10. Richey, C. R. and D. E. Davenport. Comparison Between Exponential Pile and Critical HW-Pile Buckling Measurements, HW-34643. January 26, 1955. (SECRET).

11. Richey, C.R. Unclassified Buckling Measurements Completed by the Reactor Physics Unit, HW-45654. September 24, 1956. 


\section{QUARTERLY REPORTS}

A. Physics Unit

12. October, November, December, 1953, HW-30508. (SECRET).

13. January, February, March, 1954, HW-31351. (SECRET).

14. April, May, June, 1954, HW-32349. (SECRET).

15. July, August, September, HW-33384. (SECRET).

B. Physics Research

16. October, November, December, 1954. HW-34509. (SECRET).

17. January, February, March, 1955, HW-36216. (SECRET).

18. April, May, June, 1955, HW-38202. (SECRET).

19. July, August, September, 1955. HW-40345.

20. October, November, December, 1955, HW-42182. (CONFIDENTIAL).

21. January, February, March, 1956, HW-43441.

22. April, May, June, 1956, HW-44525.

C. Nuclear Physics Research

23. July, August, September, 1956, HW-47012.

24. October, November, December, 1956, HW-48893.

25. January, February, March, 1957, HW-50598.

26. Apri1, May, June, 1957, HW-51983.

27. July, August, September, 1957, HW-53492.

28. October, November, December, 1957, HW-54591.

29. January, February, March, 1958, HW-55879.

30. April, May, June, 1958, HW-56919.

31. July, August, September, 1958, HW-57861.

32. October, November, December, 1958, HW-59126.

33. January, February, March, 1959, HW-60220.

34. Apri1, May, June, 1959, HW-61181.

35. July, August, September, 1959, HW-62727.

36. October, November, December, 1959, HW-63576.

37. January, February, March, 1960, HW-64866.

38. April, May, June, 1960, HW-66215.

39. July, August, September, 1960, HW-67219.

40. October, November, December, 1960, HW-68389.

11. January, February, March, 1961, HW-69475. 


\section{MONTHLY REPORTS}

A complete listing is not provided since the monthly reports are informal and almost all of the data are available in the Quarterly Reports. However, an indication of the titles used for reports including exponential pile work, and the periods covered, is included below as an aid in literature searching.

October, 1945, through April, 1952: 100 Areas Technical Activities Report Physics

May, 1952, through November, 1952: Technical Activities Report - Physics Research

December, 1952, through September, 1953: Technical Activities Report, Physics Unit - Applied Research Sub-Section

October, 1953, through August, 1956: Unpublished

September, 1956, to present: Physics and Instruments Research and Development Monthly Report

May, 1958, to December, 1958: Nuclear Physics Research Operation Monthly Report

(Condensed version included in preceding report) 


\section{INTERNAL DISTRIBUTION}

\section{Copy Number}

$\begin{array}{ll}1 & \text { R. A. Bennett } \\ 2 & \text { C. E. Bowers } \\ 3 & \text { C. L. Brown } \\ 4 & \text { J. H. Brown } \\ 5 & \text { W. L. Bunch } \\ 6 & \text { G. J. Busselman } \\ 7 & \text { J. L. Carter, Jr. } \\ 8 & \text { E. D. Clayton } \\ 9 & \text { D. L. Condotta } \\ 10 & \text { F. G. Dawson, Jr. } \\ 11 & \text { M. R. Egan } \\ 12-16 & \text { G. W. R. Endres } \\ 17 & \text { F. C. Engesser } \\ 18 & \text { J. E. Faulkner } \\ 19 & \text { G. C. Fullmer } \\ 20 & \text { P. F. Gast } \\ 21 & \text { R. O. Gumprecht } \\ 22 & \text { R. E. Heineman } \\ 23 & \text { W. B. Henderson } \\ 24 & \text { H. L. Henry, Jr. } \\ 25 & \text { B. R. Leonard } \\ 26 & \text { J. R. Lilley } \\ 27 & \text { R. C. Lloyd } \\ 28 & \text { C. W. Lindenmeier } \\ 29 & \text { R. H. Masterson } \\ 30 & \text { R. H. Meichle } \\ 31 & \text { C. Metelmann } \\ 32 & \text { D. I. Monnie } \\ 33 & \text { P. F. Nichols } \\ 34 & \text { R. Nilson } \\ 35 & \text { G. F. Owsley } \\ 36 & \text { E. G. Peterson } \\ 37 & \text { R. E. Peterson } \\ 38 & \text { T. J. Powell } \\ 39 & \text { W. A. Reardon } \\ 40 & \text { J. J. Regimbal } \\ 41 & \text { C. R. Richey } \\ 42 & \text { L. C. Schmid } \\ 43-45 & \text { R. K. Sharp } \\ 46 & \text { R. I. Smith } \\ 47 & \text { R. E. Tiller } \\ 48-67 & \text { D. E. Wood } \\ 68 & \text { W. K. Woods } \\ 69 & \text { J. R. Worden } \\ 70-74 & \text { 300 Files } \\ 75 & \text { Record Center } \\ 76-79 & \text { G. E. Technical Data Center, Schenetady } \\ & \text { F. }\end{array}$


EXTERNAL DISTRIBUTION (Special)

No. of Copies

G. R. Gallagher, AEC-HOO

1

A. T. Gifford, AEC-HOO

HOO Technical Information Library 
EXTERNAL DISTRIBUTION

Number of Copies

Aberdeen Proving Ground

Aerojet-General Corporation

Aerojet-General Nucleonics

Aeronautical Systems Division

Air Force Special Weapons Center

Alco Products, Inc.

Allis-Chalmers Manufacturing Company

Allis-Chalmers Manufacturing Company, Washington

Allison Division-GMC

Argonne Cancer Research Hospital

Argonne National Laboratory

Armour Research Foundation

Army Ballistic Missile Agency

Army Chemical Center

Army Environmental Hygiene Agency

Army Signal Research and Development Laboratory

Atomic Bomb Casualty Commission

AEC Scientific Representative, France

AEC Scientific Representative, Japan

Atomic Energy Commission, Washington

Atomic Energy of Canada Limited

Atomics International

Babcock and Wilcox Company

Battelle Memorial Institute

Brookhaven National Laboratory

Bureau of Ships (Code 1500)

Bureau of Yards and Docks

Carnegie Institute of Technology

Chicago Patent Group

Columbia University (Havens)

Columbia University (SOO-187)

Combustion Engineering, Inc.

Combustion Engineering, Inc. (NRD)

Convair Division, Fort Worth

Convair Division, San Diego (BUWEPS)

Defence Research Member

Defense Atomic Support Agency, Washington

duPont Company, Aiken

duPont Company, Wilmington

Edgerton, Germeshausen and Grier, Inc., Las Vegas

Frankford Arsenal

Franklin Institute of Pennsylvania

General Atomic Division

General Electric Company (ANPD)

General Electric Company, St. Petersburg

General Nuclear Engineering Corporation

Gibbs and Cox, Inc.

Glasstone, Samuel

Goodyear Atomic Corporation

Hughes Aircraft Company 
EXTERNAL DISTRIBUTION (contd.)

Number of Copies

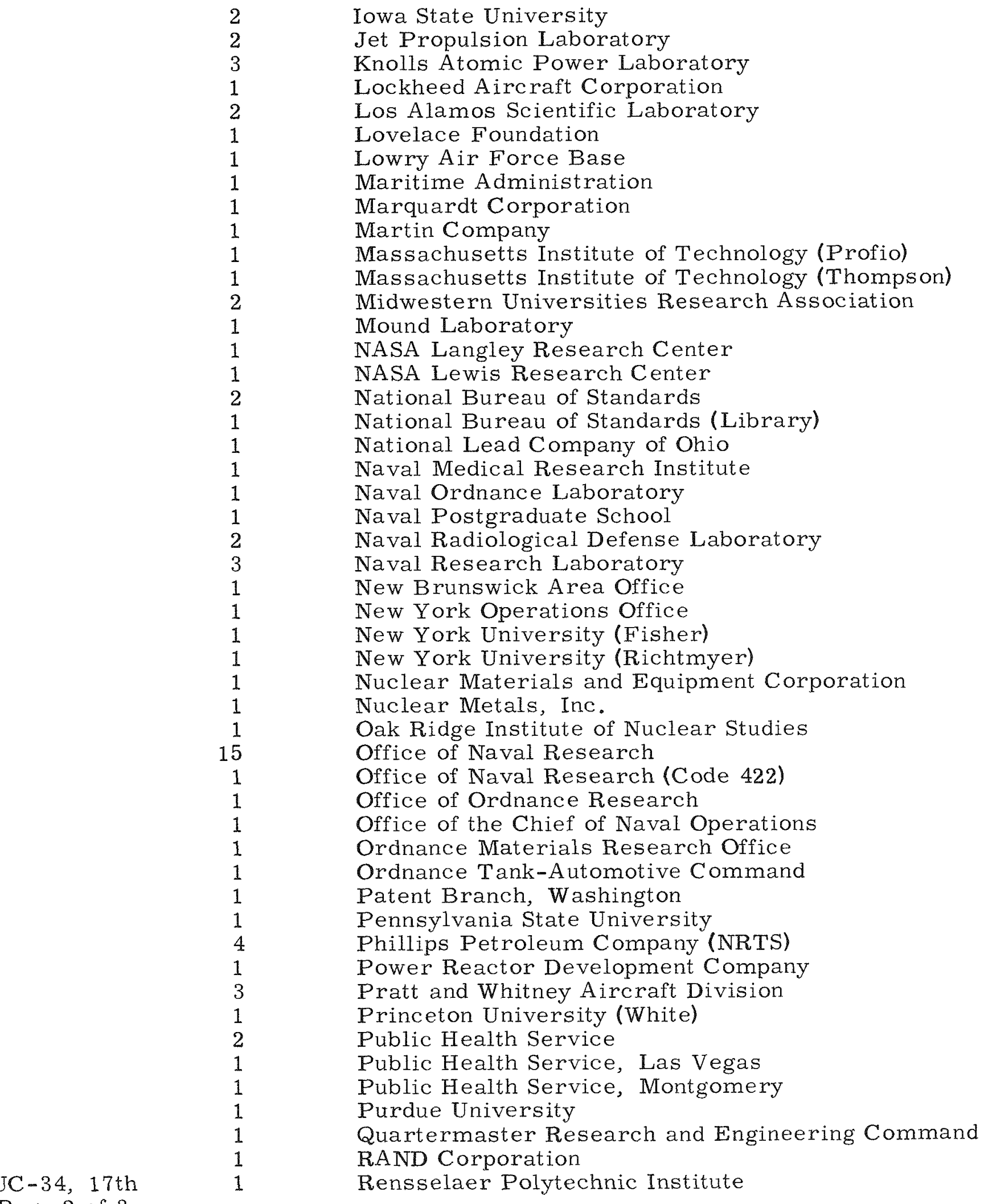


EXTERNAL DISTRIBUTION (contd.)

Number of Copies

\begin{tabular}{ll}
1 & Sandia Corporation, Albuquerque \\
1 & Sandia Corporation, Livermore \\
1 & States Marine Lines, Inc. \\
1 & Stevens Institute of Technology \\
1 & Strategic Air Command \\
1 & Surgeon General \\
1 & Sylvania Electric Products, Inc. \\
1 & Technical Research Group \\
1 & Tennessee Valley Authority \\
1 & Texas Nuclear Corporation \\
2 & Union Carbide Nuclear Company (ORGDP) \\
1 & Union Carbide Nuclear Company (ORNL) \\
2 & Union Carbide Nuclear Company (Paducah Plant) \\
1 & United Nuclear Corporation (NDA) \\
1 & U. S. Coast and Geodetic Survey, Washington \\
1 & U. S. Geological Survey, Denver \\
1 & U. S. Geological Survey, Menlo Park \\
1 & U. S. Geological Survey, Naval Gun Factory \\
1 & U. S. Geological Survey, Washington \\
2 & U. S. Patent Office \\
4 & University of California, Berkeley \\
1 & University of California, Livermore \\
1 & University of California, Los Angeles \\
1 & University of California, San Francisco \\
1 & University of Puerto Rico \\
2 & University of Rochester \\
1 & University of Rochester (Marshak) \\
1 & University of Washington (Geballe) \\
1 & University of Washington (Rohde) \\
1 & Walter Reed Army Medical Center \\
4 & Watertown Arsenal \\
2 & Westinghouse Bettis Atomic Power Laboratory \\
1 & Westinghouse Electric Corporation \\
1 & Yale University Breit) \\
1 & Yale University (Schultz) \\
& Yankee Atomic Electric Company \\
& Division of Technical Information Extension \\
\hline 5 & Office of Technical Services, Washington \\
&
\end{tabular}

$\mathrm{UC}-34,17^{\text {th }}$

Page 3 of 3 